San Jose State University

SJSU ScholarWorks

Master's Theses

Master's Theses and Graduate Research

Fall 2010

\title{
Organocatalytic decomposition of poly(ethylene terephthalate) using triazabicyclodecene proposal
}

Julien Matsumoto Lecuyer

San Jose State University

Follow this and additional works at: https://scholarworks.sjsu.edu/etd_theses

\section{Recommended Citation}

Lecuyer, Julien Matsumoto, "Organocatalytic decomposition of poly(ethylene terephthalate) using triazabicyclodecene proposal" (2010). Master's Theses. 3873.

DOI: https://doi.org/10.31979/etd.c3kc-xcdz

https://scholarworks.sjsu.edu/etd_theses/3873

This Thesis is brought to you for free and open access by the Master's Theses and Graduate Research at SJSU ScholarWorks. It has been accepted for inclusion in Master's Theses by an authorized administrator of SJSU ScholarWorks. For more information, please contact scholarworks@sjsu.edu. 


\title{
ORGANOCATALYTIC DECOMPOSITION OF POLYETHYLENE TEREPHTHALATE USING TRIAZABICYCLODECENE
}

\author{
A Thesis \\ Presented to \\ The Faculty of the Department of Chemical and Materials Engineering \\ San Jose State University \\ In Partial Fulfillment of \\ the Requirements for the Degree \\ Master of Science
}

by

Julien Matsumoto Lecuyer

December 2010 
(C) 2010

Julien Matsumoto Lecuyer ALL RIGHTS RESERVED 


\title{
ORGANOCATALYTIC DECOMPOSITION OF POLYETHYLENE
}

TEREPHTHALATE USING TRIAZABICYCLODECENE

\author{
by \\ Julien Lecuyer \\ APPROVED FOR THE DEPARTMENT OF CHEMICAL \& MATERIALS \\ ENGINEERING

\section{SAN JOSÉ STATE UNIVERSITY} \\ August 2010 \\ Dr. Melanie McNeil \\ Department of Chemical \& Materials Engineering \\ Dr. Richard Chung \\ Department of Chemical \& Materials Engineering \\ Dr. James Hedrick \\ IBM Almaden Research Center \\ Dr. Kazuki Fukushima \\ IBM Almaden Research Center
}




\section{ABSTRACT \\ ORGANOCATALYTIC DECOMPOSITION OF POLYETHYLENE TEREPHTHALATE USING TRIAZABICYCLODECENE}

This study focuses on the organocatalytic decomposition of polyethylene terephthalate (PET) using 1,5,7-triazabicyclo[4.4.0]dec-5-ene (TBD) to form a diverse library of aromatic amides. The reaction scheme was specifically designed to use low reaction temperatures $\left(>150^{\circ} \mathrm{C}\right)$ and avoid using solvents during the reaction to provide a more environmentally friendly process. Of all the amines tested, PET aminolysis with aliphatic and aromatic amines demonstrated the best performance with yields higher than 72\%. PET aminolysis with click functionalized and non-symmetric reagents facilitated attack on certain sites on the basis of reactivity. Finally, the performance of the PET degradation reactions with secondary amine and tertiary amine functionalized reagents yielded mixed results due to complications with isolating the product from the crude solution. Four of the PET-based monomers were also selected as modifiers for epoxy hardening to demonstrate the ability to convert waste into monomers for high-value applications. The glass transition temperatures, obtained using differential scanning calorimetry (DSC) and dynamic mechanical analysis (DMA) of the epoxy composite samples treated with the PET-based monomers, were generally higher in comparison to the samples cured with the basic diamines due to the hydrogen bonding and added rigidity from the aromatic amide group. Developing these monomers provides a green and commercially viable alternative to eradicating a waste product that is becoming an environmental concern. 


\section{ACKNOWLEDGEMENTS}

First and foremost, I would like to sincerely thank Dr. Jim Hedrick and Dr. Kazuki Fukushima for providing me the opportunity to work on such a remarkable project. I cannot explain how much their humor, patience, and willingness to help has meant to me; for that, I will forever be in their debt. I would also like to thank Dr. Melanie McNeil, my advisor, and Dr. Richard Chung for helping me reach my potential as an engineer through the completion of this project. In addition, I would like to thank the following people for their appreciated contributions to this project: Dr. Teddie Magbitang, Dr. Dolores Miller, Dr. Joseph Sly, Dr. Young-Hye Na, Dr. Hans Horn, Dr. Julia Rice, Dr. Robert Allen, Dr. Robert Miller, Victor Lee, Neil Peters, and the members of KACST. I also owe a great deal of gratitude toward San Jose State University and IBM Almaden Research Center for providing me with the environment necessary for me to succeed.

Finally, I would like to dedicate this thesis to my parents. I would not be where I am today without their emotional and financial support, especially during my years as an undergraduate and graduate student. I will never be able to thank them enough for everything they have done for me. 


\section{TABLE OF CONTENTS}

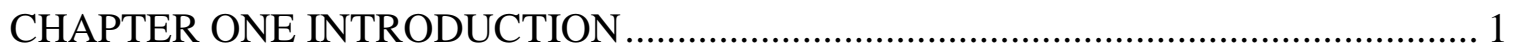

Green Chemistry............................................................................. 1

$1.2 \quad$ Production of Polyethylene Terephthalate ......................................... 3

Recycling of Polyethylene Terephthalate................................................. 5

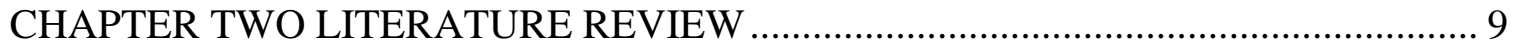

Evolution of Organocatalysts ........................................................... 10

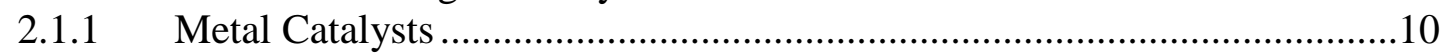

2.1.2 Pyridine-based and Imidazolidone-based Organocatalysts ........................11

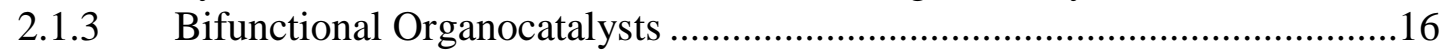

2.1.4 Amidine-based and Guanidine-based Organocatalysts ..............................18

2.2 Applications of Aromatic Amide/Ester Compounds.................................. 22

2.2.1 Epoxy Hardening ..........................................................................23

2.2.2 Interfacial Polymerization and Desalination Membranes ..........................27

2.2.3 Applications in Specialty Polymers ...................................................29

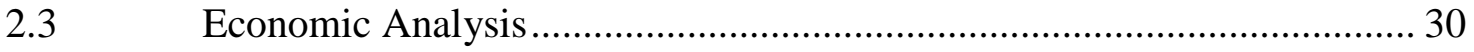

2.3.1 Economics of PET Degradation Via Organocatalysis .............................. 31

2.3.2 Economics of Incorporating PET-derived Diamines for Epoxy

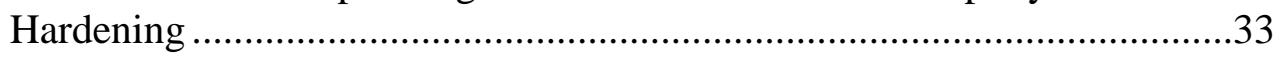

Literature Review Summary .......................................................... 34

CHAPTER THREE PROJECT OBJECTIVES ....................................................... 36

CHAPTER FOUR MATERIALS AND METHODS ................................................. 38

4.1 Organocatalytic Decomposition of Polyethylene Terephthalate ............... 38

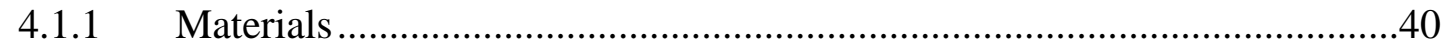

4.1.2 Experimental Procedure for PET Decomposition ......................................41

4.1.3 PET Decomposition Incorporating Reused Reagents ................................53

4.2 Epoxy Hardening Process Incorporating PET-derived Amides ................. 54

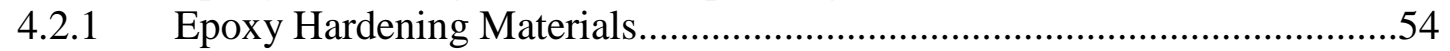

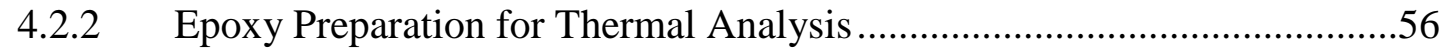

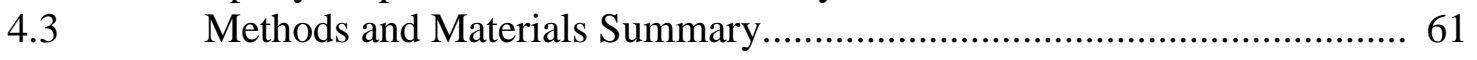


5.1 H-NMR Analysis for PET-Derived Monomers ......................................... 64

5.2 Performance Analysis of PET Aminolysis Reactions ............................... 65

5.2.1 PET Aminolysis with Aliphatic and Aromatic-Containing Diamines ........65

5.2.2 PET Aminolysis with Non-Symmetric Amines ...........................................68

5.2.3 PET-Aminolysis with Click-Functionalized and Secondary Amines ..........70

5.2.4 PET Aminolysis with Tertiary-Functionalized Amines .................................72

5.3 Recycling of Amine Reagents for PET-Aminolysis ................................. 75

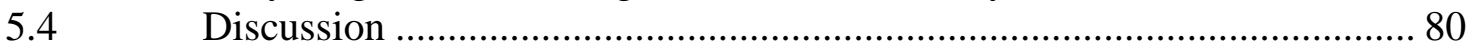

5.4.1 Environmental Significance of Developing PET-Based Monomers............80

5.4.2 Process Optimization................................................................................ 81

5.4.3 Importance of Properties PET-Based for Potential Applications..................82

CHAPTER SIX PET-DIAMINE RESULTS AS EPOXY HARDENING

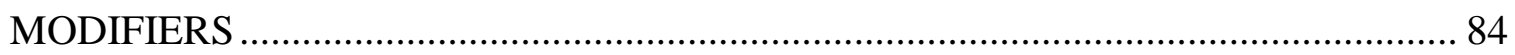

6.1 Differential Scanning Calorimeter Results................................................. 85

6.2 Dynamic Mechanical Analysis Results .................................................. 88

6.2.1 PETED Composite DMA Results ...........................................................89

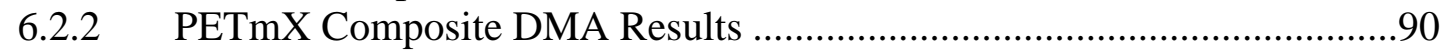

6.2.3 PETDAMDPA Composite DMA Results ..................................................92

6.2.4 PETDETA Composite DMA Results ..........................................................93

CHAPTER SEVEN CONCLUSIONS AND FUTURE WORK ………………….......... 95

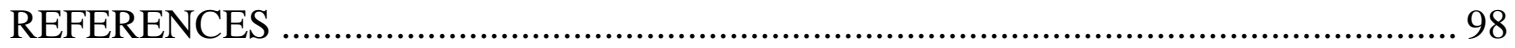




\section{LIST OF TABLES}

Table 1. 12 principles of green chemistry [2]

Table 2. Alterations of the chemical structure of DMAP and its effect on the acylation of 1-methylcyclohexanol with acetic anhydride [19]

Table 3. N-heterocyclic carbene structure dependence on the transesterification of methyl benzoate and various alcohols [22]

Table 4. Performance of different organocatalysts on the transesterification of methyl acetate with benzyl alcohol [24].

Table 5. Performance of reverse osmosis membranes with monomeric aliphatic diamines and terephthaloyl chloride [45].

Table 6. Current prices for PET and chemicals required for organocatalytic recycling...31

Table 7. Economic analysis of synthesizing PET from oil and from organocatalytic recycling.

Table 8. Economic analysis of implementing PET-decomposed polyamides for epoxy hardening applications.

Table 9. Potential reagents for PET decomposition and modification.

Table 10. General strategy for selecting temperature for the reaction

Table 11. List of reaction temperatures for various reagents in the decomposition of PET.

Table 12. Experimental design for epoxy hardening of PET-derived diamines for DSC.

Table 13. Experimental design for epoxy hardening of PET-derived diamines for DSC

Table 14. Summary of PET aminolysis reactions

Table 15. Table of $\mathrm{Tg}_{\infty}$ values of cure epoxy composite with $100 \%$ PET-diamine .88 


\section{LIST OF FIGURES}

Figure 1. Decennial production of municipal solid waste generation in the United

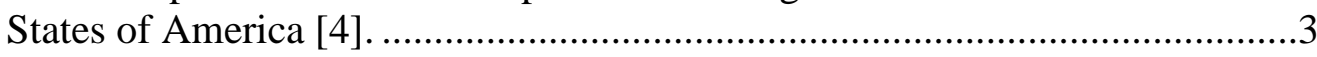

Figure 2. General schematic of the polymerization process of PET....................................3

Figure 3. Annual production and storage of plastic bottles in the U.S. (Adapted from National Association for PET Container Resources) [6]...................................4

Figure 4. Distribution of recycled PET used for secondary applications [11]...................6

Figure 5. Chemical recycling of PET using methanolysis [12]........................................6

Figure 6. Illustration of 1,5,7-triazabicyclo[4.4.0]dec-5-ene (TBD) ................................

Figure 7. Coordination-Insertion mechanism of an organometallic ion on lactic acid

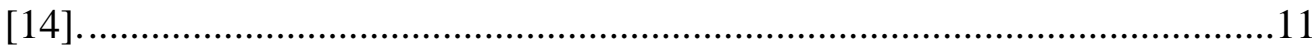

Figure 8. Illustration of pyridine-derivative 4-Dialkylaminopyridine (DMAP)..............12

Figure 9. Catalytic pathway of DMAP in the acetylation of an alcohol with acetic anhydride [20].........................................................................................

Figure 10. An illustration of $n$-heterocyclic carbene, a derivative of imidazolidone. ......14

Figure 11. Illustration of BDCT and bidente hydrogen bonding formed due to

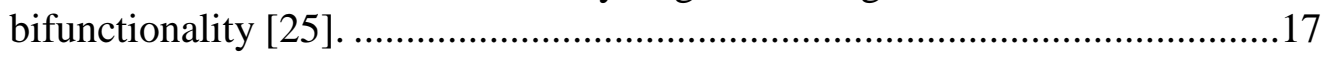

Figure 12. Illustration of amidine-derived organocatalysts............................................18

Figure 13. An illustration of guanidine-based organocatalyst TBD................................19

Figure 14. Catalytic mechanisms for the ring-opening polymerization of t-lactide

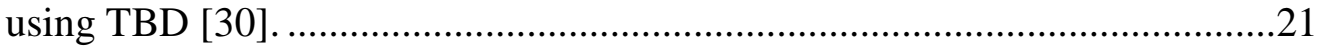

Figure 15. Aminolysis of poly(ethylene terephthalate) with diethylenetriamine [36]......21

Figure 16. Time-temperature transformation profile for thermosetting plastics [37].......23

Figure 17. Cross-linking polymerization through epoxy hardening using BADGE and various polyamine/polyamides. 
Figure 18. Implementing an aromatic amide as a fortifier for the epoxy hardening, using methylene dianiline as the copolymer.

Figure 19. Click chemistry reaction illustration between an azide and an alkyl group [51]......

Figure 20. Illustration of the experimental apparatus set-up for PET-degradation reaction.

Figure 21. PET degradation reaction conditions using ethylenediamine reagent.

Figure 22. Selected PET-derived diamines used for epoxy hardening applications .55

Figure 23. Illustration of epoxy hardened polymer for DSC analysis. .57

Figure 24. Glass transition temperature at fully cured state for DSC analysis. .58

Figure 25. DMA epoxy samples (a) after sample preparation and (b) post-cured. .60

Figure 26. Expected DMA result for fully cured epoxy polymer

Figure 27. H-NMR spectrum of PETED used to illustrate the characteristic peaks specific to the aromatic amide group for each PET-based monomer.

Figure 28. H-NMR characterization of the aliphatic PET-based diamines: (a) PETED, (b) PETDAB, and (c) PETDAH.

Figure 29. H-NMR characterization of the phenyl containing PET-based diamines:

(a) PETmX, (b) PETpX, (c) PETABA, and (d) PETPPD. .68

Figure 30. H-NMR characterization of non-symmetric PET-based monomers: (a) PETEthA, (b) PETAEP, and (c) PETDETA.

Figure 31. H-NMR characterization of PET-based monomers with clickfunctionalization: (a) PETAllyl and (b) PETPro. .71

Figure 32. H-NMR characterization of PET-based monomers using secondary amines: (a) PETPPI and (b) PETPPA. .71

Figure 33. H-NMR characterization of PET-based monomers with tertiaryfunctionalized amines: (a) PETDAMDPA, (b) PETDAP, and (c) PETTAEA. .74

Figure 34. H-NMR spectrum for PETDAMDPA (dimer). .75 
Figure 35. Reaction scheme for using recycled $\mathrm{mX}$ to perform a PET aminolysis reaction

Figure 36. NMR analysis of PETmX product using (1) pristine $\mathrm{mX}$ and (2) recycled $\mathrm{mX}$

Figure 37. Reaction scheme for using recycled ED to perform a PET aminolysis reaction.

Figure 38. NMR analysis of PETED product using (1) pristine ED and (2) recycled $\mathrm{ED}$

Figure 39. PET-derived compounds used as modifiers for epoxy hardening. .84

Figure 40. DSC analysis of cured epoxy polymer (100\% PETED) heated and cured several times to reach $\mathrm{Tg}_{\infty}$

Figure 41. DSC analysis for epoxy hardened polymers using PET-derived diamines.....87

Figure 42. DMA analysis for epoxy curing with (a) Ethylenediamine and (b) PETEthylenediamine.

Figure 43. DMA analysis for epoxy curing with (a) m-xylylenediamine and (b) PETm-Xylylenediamine.

Figure 44. DMA analysis for epoxy curing with (a) Diamino-N-methyldipropylamine and (b) PET-Diamino-N-methyldipropylamine.

Figure 45. DMA analysis for epoxy curing with (a) Diethylenetriamine and (b) PETDiethylenetriamine. 


\section{CHAPTER ONE}

\section{INTRODUCTION}

Dramatic increases in technology over the last century have led to improvements in productivity and efficiency throughout various industrial sectors. The ability to produce manufactured goods at a significantly higher level has adversely placed a significant toll on the environment. Governments throughout the world have become increasingly aware of the pollution and waste caused by the industrial sector. The Kyoto Protocol demonstrates a worldwide collaboration in response to the environmental impact caused by escalating $\mathrm{CO}_{2}$ emissions [1].

\subsection{Green Chemistry}

Over the last two decades, there has been a growing emphasis on environmental concerns and policies focused on solving environmental problems. Starting with the Pollution Prevention Act of 1990, the government has had a direct role in establishing the idea of "green chemistry" in research as well as in industry [2]. The EPA has accepted the 12 principles of green chemistry, formulated by Anastas and Kirchoff, as the set objectives by which to define a process as green chemistry (Table 1). Current research implements the ideas of green chemistry into serviceable processes. For example, Jeon et $a l$. were able to demonstrate higher yields using solvent-free catalysis with bis(sulfonamide) diol ligand as a catalyst in a titanium tetraisopropoxide and dialkylzinc reaction medium for the addition of dialkylzinc to ketones [3]. The ability of this method to reduce solvents while improving conversion through catalysis demonstrates an 
environmentally and economically sound method of performing enantioselective reactions. Jeon et al. improved a current industrially practiced process by fulfilling not just one, but a combination of the 12 principles of green chemistry (Table 1). These changes can make current processes into ones that are more environmentally and economically benign.

Table 1. 12 principles of green chemistry [2].

\begin{tabular}{|c|c|}
\hline $\begin{array}{l}\text { 1. Better to prevent waste than to } \\
\text { clean it up after formation. }\end{array}$ & $\begin{array}{l}\text { 7. Raw materials should be focused } \\
\text { on renewable resources. }\end{array}$ \\
\hline $\begin{array}{l}\text { 2. Maximize utilization of materials } \\
\text { for synthesis. }\end{array}$ & $\begin{array}{l}\text { 8. Unnecessary derivatization (i.e., } \\
\text { blocking groups) should be } \\
\text { avoided. }\end{array}$ \\
\hline $\begin{array}{l}\text { 3. Minimize the use and production } \\
\text { of hazardous material. }\end{array}$ & $\begin{array}{l}\text { 9. Catalytic reagents should be used } \\
\text { over stoichiometric reagents. }\end{array}$ \\
\hline $\begin{array}{l}\text { 4. Increase efficacy while reducing } \\
\text { toxicity. }\end{array}$ & $\begin{array}{l}\text { 10. End life of chemicals should not be } \\
\text { persistent in the environment. }\end{array}$ \\
\hline $\begin{array}{l}\text { 5. Prevent the use of auxiliary } \\
\text { substances (i.e., solvents). }\end{array}$ & $\begin{array}{l}\text { 11. Procedure of formation of toxic } \\
\text { substances should be fully } \\
\text { analyzed. }\end{array}$ \\
\hline $\begin{array}{l}\text { 6. Minimize energy requirements } \\
\text { using ambient conditions. }\end{array}$ & $\begin{array}{l}\text { 12. Minimize substances with potential } \\
\text { for accidents }\end{array}$ \\
\hline
\end{tabular}

The Environmental Protection Agency (EPA) data reveal the total municipal solid waste generated nationally every decade (Figure 1) [4]. The data demonstrate a growing trend in total generation of waste as well as generation per capita. As a result, environmental-friendly processes, such as recycling, are being implemented to reduce the amount of waste sent to landfills. 


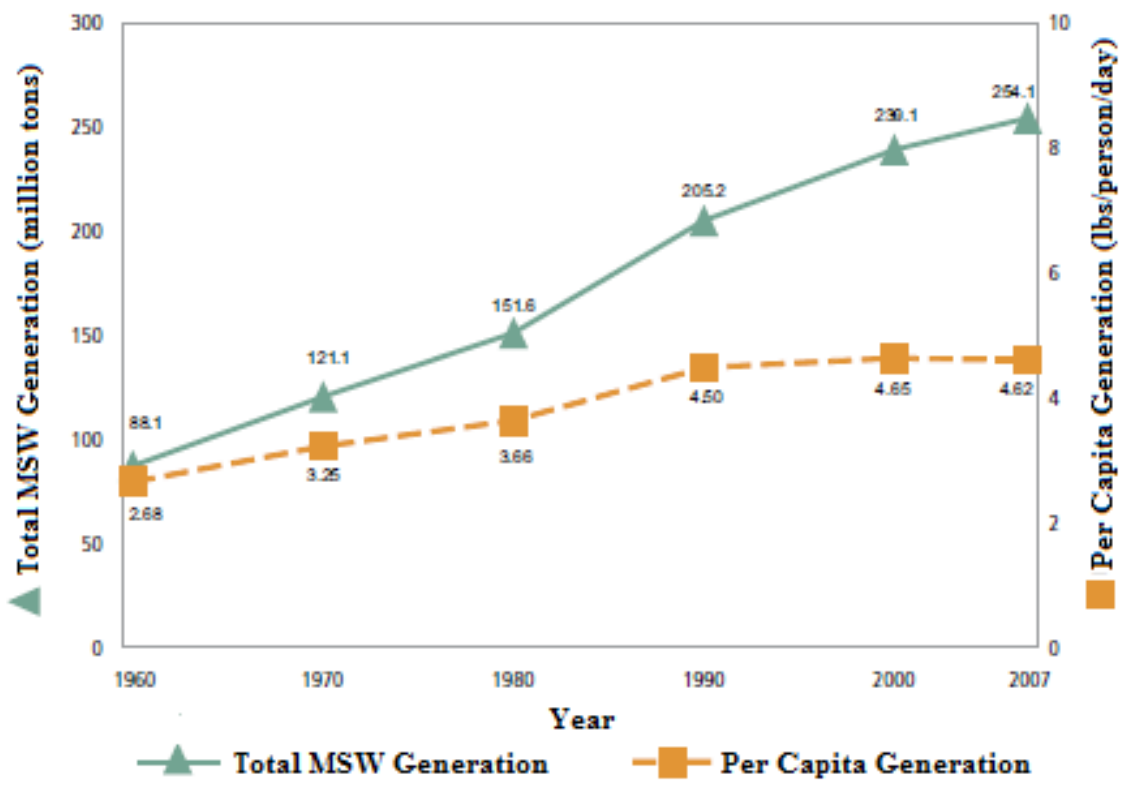

Figure 1. Decennial production of municipal solid waste generation in the United States of America [4].

\subsection{Production of Polyethylene Terephthalate}

The growing beverage industry has been an active contributor to the increasing quantity of municipal solid waste. Polyethylene terephthalate (PET), the main constituent in plastic bottles, is simple to synthesize using solid state polymerization, reacting terephthalic acid with ethylene glycol using metal catalysts such as antimony [5]. Figure 2 illustrates the general reaction from the oil-based derivatives to the polymer.

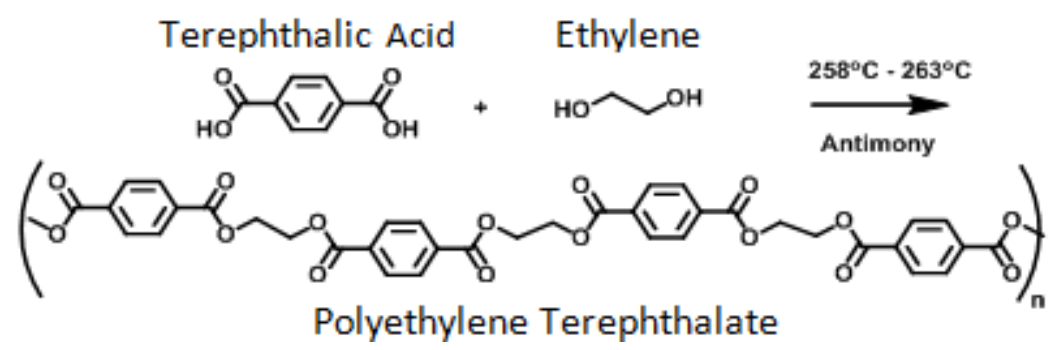

Figure 2. General schematic of the polymerization process of PET. 
Combining the simplicity of the polymerization process with the durable mechanical properties of PET, industries throughout the world have used PET as the staple polymer for beverage packaging. As a result, the accumulation of plastic bottles in the U.S., as illustrated in Figure 3, has led to a growing concern over the treatment and disposal of generated waste, as well as the potential negative effects in the environment [6]. The environmental impact of plastic waste generation has troubled many researchers, especially given waste accumulation in the North Pacific Gyre. The polymeric waste that grows in the Pacific, which transports persistent organic pollutants such as polychlorinated biphenyl, has had detrimental effects on the ecosystem $[7,8]$. In response to the growing production of plastic bottles, recycling has played a major role in reducing waste by converting used bottles into alternative products.

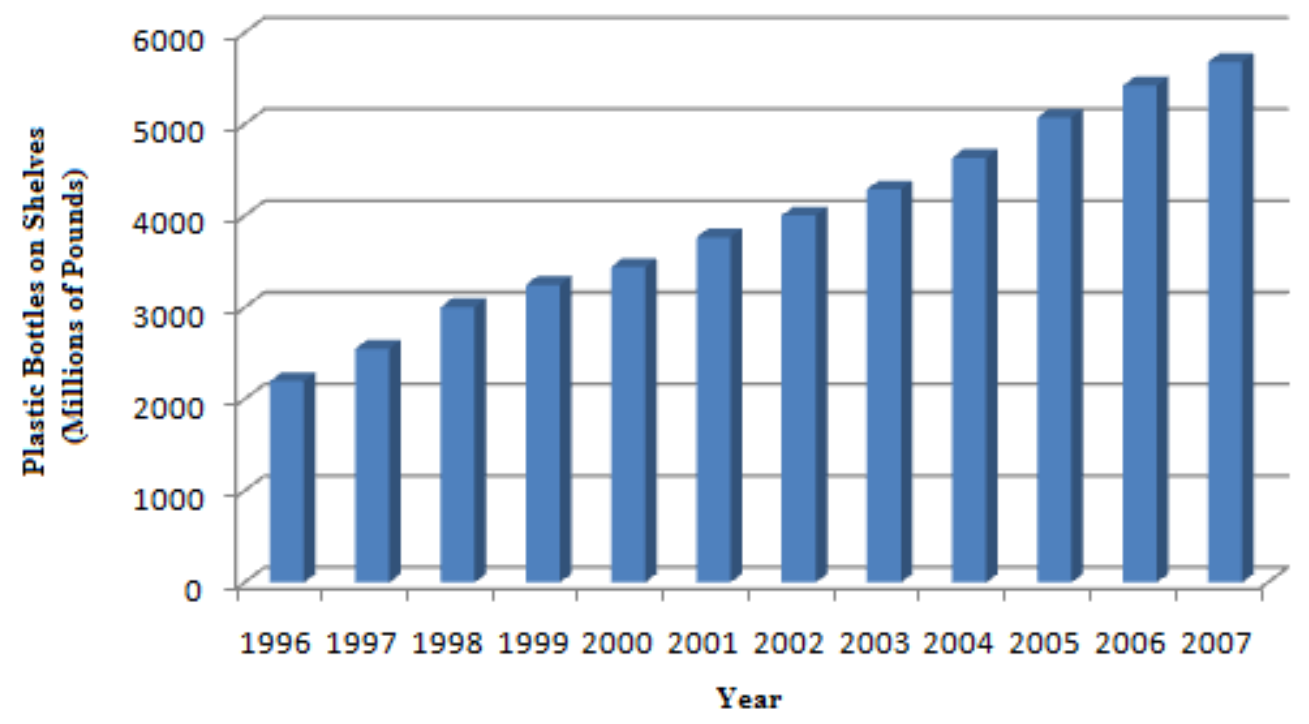

Figure 3. Annual production and storage of plastic bottles in the U.S. (Adapted from National Association for PET Container Resources) [6]. 


\subsection{Recycling of Polyethylene Terephthalate}

Currently, the dominant form of PET recycling worldwide is mechanical recycling. Mechanical recycling consists of grinding PET bottles into powder using physical means as opposed to chemical means. The general process for mechanical recycling involves a six-stage process: sorting, granulation, flotation, washing, drying, and extrusion [9]. Because PET is degraded using physical means, the powder gathered from mechanical recycling does not share the same quality properties as virgin PET. Therefore, mechanically recycled PET cannot be reused in plastic bottle production due to the diminished mechanical properties of PET through physical degradation [10]. Instead, these recycled products have been commercially produced to be used as fibers in a large variety of secondary applications varying from clothing to carpeting [11]. Figure 4 displays the distribution of recycled PET used in secondary applications in the United States. Mechanical recycling, however, does not prevent the accumulation of PET in landfills; the lifetime of the secondary product is limited, and it will eventually be discharged into the landfill. Despite this fact, mechanical recycling is an inexpensive way to reduce the buildup of waste PET into our landfills after they have been consumed. 


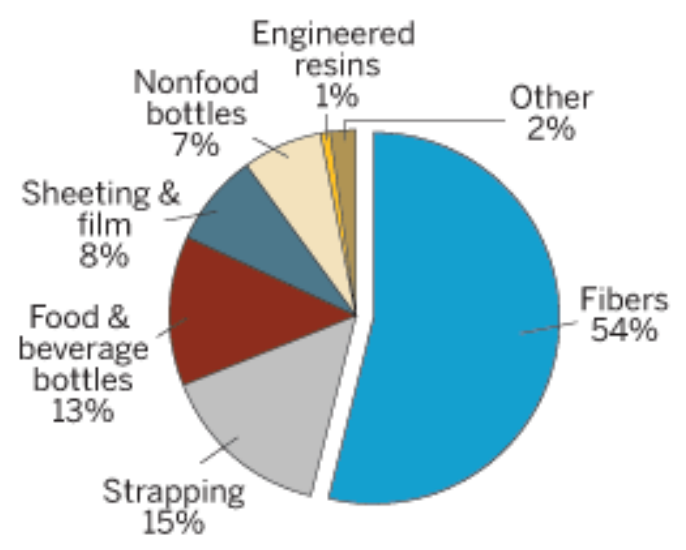

2005 U.S. market $=864$ million $\mathrm{lb}$

Figure 4. Distribution of recycled PET used for secondary applications [11].

Chemical recycling provides an ample method of degrading PET into its monomeric form, which can then be used for the reproduction of plastic bottles. Chemical recycling cleaves the polymer at strategic points generally using methanolysis, glycolysis, or hydrolysis in order to cleave and then esterify to produce either bis(hydroxyethyl) terephthalate or terephthalic acid [12]. Figure 5 illustrates the decomposition of PET through methanolysis using zinc acetate as the main catalyst. The disadvantages of the chemical recycling process stem from the rigorous conditions required for the reaction to proceed as well given the difficulties in removing the metal catalyst from the product.

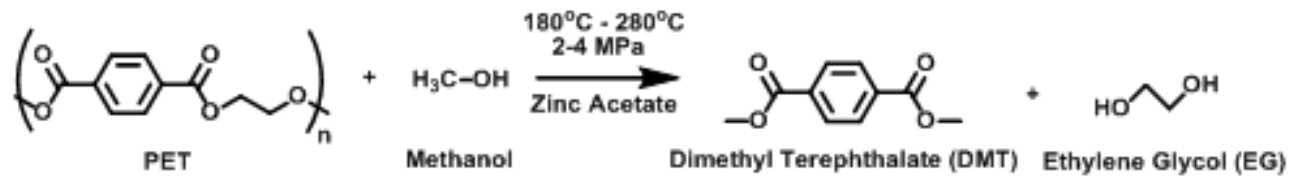

Figure 5. Chemical recycling of PET using methanolysis [12]. 
Environmentally, the amounts of chemical waste generated and large energy requirements associated with chemical recycling make mechanical recycling a more appealing choice industrially. Chemical recycling does eliminate the disposal of PET into the landfills. If the chemical process can be altered to reduce the constraints of the system, chemical recycling may become a more viable method for recycling PET.

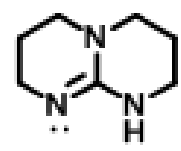

Figure 6. Illustration of 1,5,7-triazabicyclo[4.4.0]dec-5-ene (TBD).

This thesis focuses on the chemical decomposition of PET replacing currently used metal catalysts with commercially available organocatalysts to dramatically reduce the conditions required for PET degradation while maintaining a high yield. In this case, the guanidine-based 1,5,7-triazabicyclo[4.4.0]dec-5-ene (TBD) catalyst, depicted in Figure 6, is the focus of the project due to the high reactivity of TBD on ester linkages. The ability to use organocatalysts resolves several issues incorporated with using metal catalysts. Chemical recycling using organocatalysts provides not only a means for degrading PET but also is an exemplary case of meeting green chemistry standards due to its ability to drastically reduce experimental conditions, improve the efficiency of existing catalysts, and even eradicate the need for solvents. Alternatively, the degradation of PET may also lead to the chemical synthesis of new innovative chemicals for new long-term applications instead of reproduction of plastic bottles. The new 
monomers generated from the degradation of PET are identified as PET-reagent; the reagent used to modify PET is the transesterification/aminolysis agent. 


\section{CHAPTER TWO}

\section{LITERATURE REVIEW}

The increased production of PET has caused growing concern over the environmental implications of PET waste. Organocatalysis may provide a way to make current chemical recycling processes a more environmentally benign process through the applications of green chemistry. Organocatalysis has emerged largely in chemical synthesis/degradation because of the ability to strategically design these catalysts in order to improve the performance to their respective reactions. Understanding the evolution of organocatalysts reveals triazabicyclodecene (TBD) as a potent catalyst for enhancing the performance of certain specialized reactions, such as the degradation and modification of PET. In addition to catalytically degrading PET back into its basic monomers, TBD may also be able to produce PET derivatives through transesterification and aminolysis reactions that could be used for high-valued applications. This chapter presents the literature focused on the evolution of organocatalysts, as well as their application to organic synthesis and possible applications for aromatic amides formed from PET-

derived amides/esters. Also presented are the economic incentives for modifying PET compounds. 


\subsection{Evolution of Organocatalysts}

Catalysis has significantly advanced organic chemistry in both laboratory and industrial settings. By minimizing reaction conditions while improving the performance of synthesis reactions, catalysts have become a heavily studied field, with applications ranging from pharmaceuticals to industrial chemicals (i.e., petroleum) to even environmental applications. Organocatalysts represent a relatively new field of catalysts using strictly organic components to improve process conditions, while avoiding the hindrances that occur with metal constituents. A key concept in organocatalysis involves the ability to strategically modify the structures of dated organocatalysts into higher performing ones. By understanding the evolution of organocatalysis, one can determine the factors that enhance catalytic performance for the development and application of new sound organocatalysts.

\subsubsection{Metal Catalysts}

Metal catalysts have become a staple in organic chemistry due to their cationic properties, which allow them to form stable complexes with electronegative counterparts [13]. The coordination-insertion mechanism provides a prime example of how the metal ion targets the electronegative atom in the organic molecule and inserts the organic constituent of the organometallic catalyst into the ring for it to open and polymerize, as displayed in Figure 7 [14]. By using the basis of the metal-catalyzed mechanism, organocatalysts can be strategically developed to mimic or even improve current synthetic/polymerizing properties. 


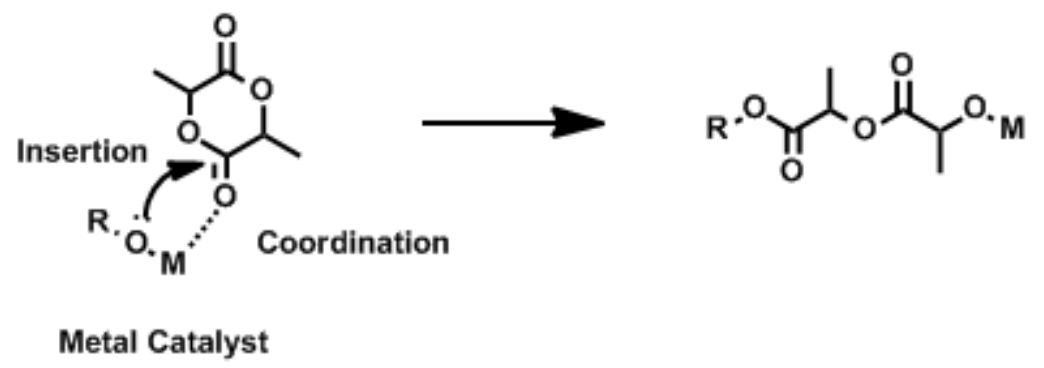

Figure 7. Coordination-Insertion mechanism of an organometallic ion on lactic acid [14].

The use of metal catalysts in esterification reactions has been well-documented. Ishihara et al. demonstrated an ester condensation reaction using hafnium and zirconium salts between equimolar ratios of carboxylic acids and alcohols [15]. Successful conversion of several different carboxylic acids and alcohols demonstrated yields of $99 \%$, illustrating the effectiveness of using cationic catalysts for esterification reactions. Similarly, Otton et al. performed the transesterification of benzoic acid with 2-butoxyethanol, varying the catalyst used for the reaction with Titanium, and demonstrating the highest conversion [16]. The demonstration of metal catalysts for the esterification of different alcohols/carboxylic acids provides a foundation for the application of organocatalysts for the same reactions.

\subsubsection{Pyridine-based and Imidazolidone-based Organocatalysts}

Similar to the mechanism for the ring opening polymerization using metal catalysts, the goal for organocatalysts is to strategically develop an organic compound for nucleophilic catalysis. Pyridine has been a staple organic compound for nucleophilic substitution, amplified by the basic effects resulting from the electron pair on the tertiary 
amine [17]. Using the pyridine molecule as a basic model, researchers can strategically devise a new molecule for nucleophilic organocatalysis.

One of the first organocatalysts, the pyridine-functionalized organocatalyst 4dialkylaminopyridine (DMAP), was discovered by Steglich and Hölfe [18]. This compound is comprised of a pyridine derivative combined with an additional tertiary amine opposite of the aromatic amine, as shown in Figure 8. The tertiary amine "tail" causes the aromatic electrons to shift toward the aromatic amine, making the lone pair on the aromatic amine more prone to nucleophilic substitution. In addition, the tertiary amine forms a complex with the negative ion formed after the nucleophilic substitution [19]. DMAP is a prime example of applying functional end groups to improve the reactivity of an organocatalyst.

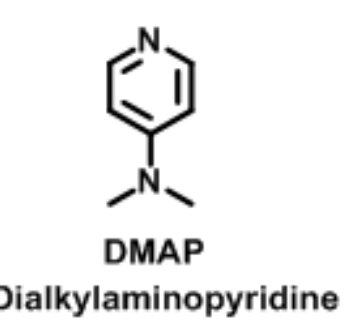

Figure 8. Illustration of pyridine-derivative 4-dialkylaminopyridine (DMAP).

The catalytic pathway of DMAP during ester reactions provides a good illustration of how strategic organocatalysts can improve the efficiency of the reaction. A mechanistic study on the acetylation of alcohols using DMAP was done by Xu et al. using computation chemistry to calculate the relative enthalpies for various complexes [20]. The results demonstrated that the most stable mechanism for the catalytic pathway involves the acylation of the acetyl group with DMAP, as shown in Figure 9. 


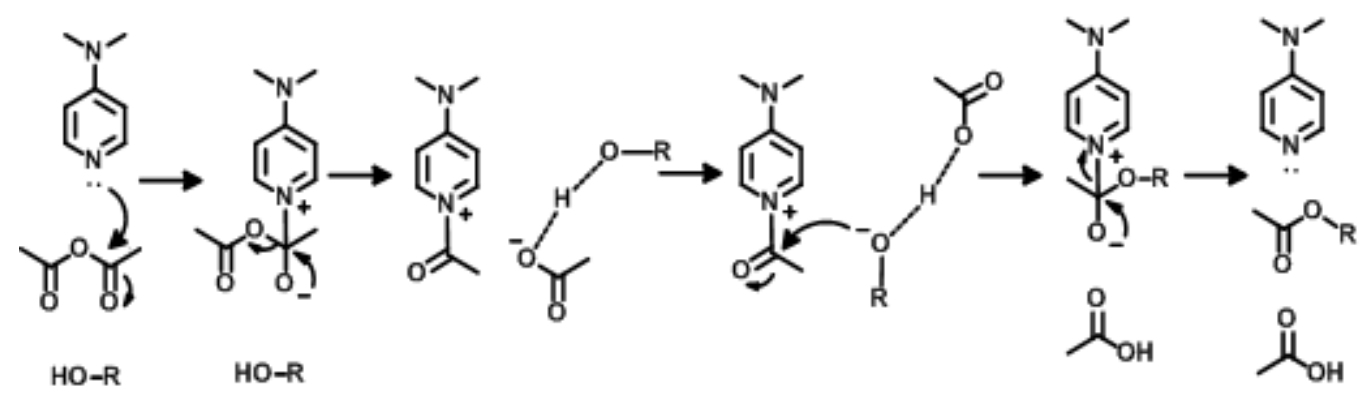

Figure 9. Catalytic pathway of DMAP in the acetylation of an alcohol with acetic anhydride [20].

By understanding the catalytic pathway of DMAP, researchers can alter the catalyst structurally in a way that can exploit the catalytic pathway of the reaction. Spivey and Arseniyadis devised an experiment altering the functional tail on DMAP and comparing the relative reactivities of the altered catalysts to determine the optimum structure [19]. Table 2 illustrates a brief variation in the chemical structure of DMAP and how reactivities vary based on structure. Through comparison of the relative reactivities, pyrrolidinopyridine (PPY) demonstrated an improvement in the acylation of 1methylcyclohexanol.

Table 2. Alterations of the chemical structure of DMAP and the structure's effect on the acylation of 1-methylcyclohexanol with acetic anhydride [19].

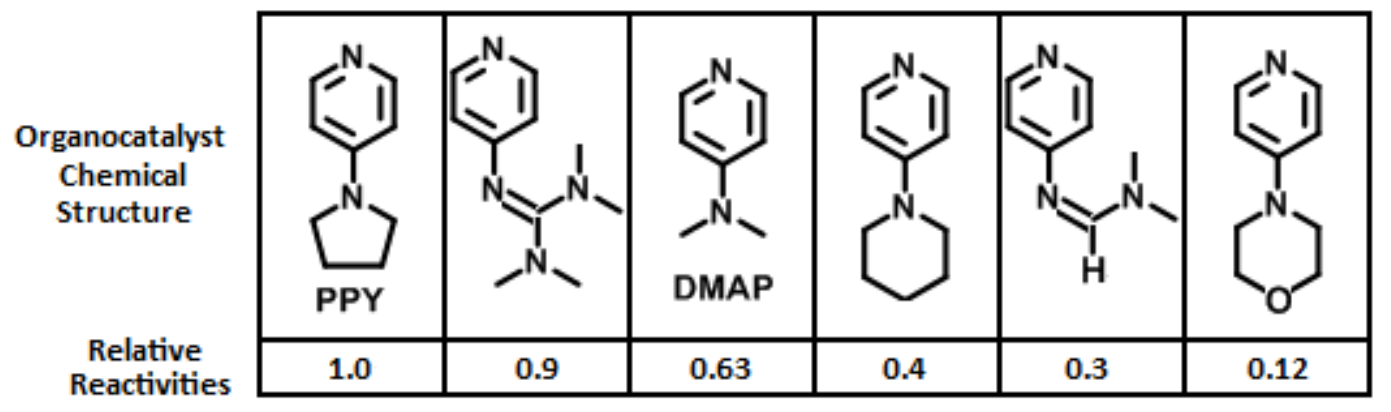


A study by Macmillen demonstrated one of the earliest involvements of imidazolidone-based organocatalysts for the improvement of the Diels-Alder reaction [21]. This new class of organocatalysts provided a new series of catalysts for the application in several chemical synthesis applications. Structurally, n-heterocyclic carbenes offer more potential for higher reactivity due to the increased nucleophilicity compared to older organocatalysts, such as DMAP [14]. Researchers have studied how matching up different functional groups with the imidazolidone-based n-heterocyclic catalyst, shown in Figure 10, affects the performance of the transesterification reaction between carbonyl groups and alcohols.

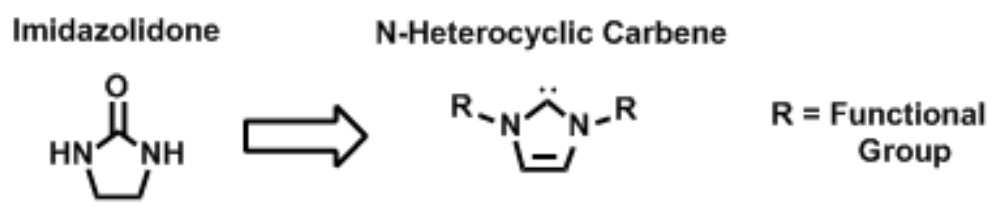

Figure 10. An illustration of n-heterocyclic carbene, a derivative of imidazolidone.

Nyce et al. studied the effect of the size of the n-heterocyclic carbenes on the transesterification of methyl benzoate with various alcohols [22]. N-heterocyclic carbenes, independent of size, demonstrated relatively high yields for the transesterification of carboxylic esters with primary alcohols. However, the conversion between carboxylic esters and secondary alcohols decreased significantly with increasing catalyst size (Table 3). Furthermore, the reaction with tertiary alcohols was nonexistent. As expected, the reactivity of the alcohol was an important aspect of the performance of the transesterification reaction. Singh et al. demonstrated successful transesterification of 
secondary, aliphatic, aromatic cyclic, and even tertiary alcohols in the presence of cyclohexyl functionalized n-heterocyclic carbenes (ICy) [23].

Table 3. N-heterocyclic carbene structure dependence on the transesterification of methyl benzoate and various alcohols [22].

\begin{tabular}{|c|c|c|c|c|}
\hline & & \multicolumn{3}{|c|}{ Alcohols } \\
\hline Abbr. & $\mathrm{N}$-Heterocyclic Carbene & Primary & $\begin{array}{c}\text { Secondary } \\
\mathrm{R}_{\mathrm{H}}^{\prime}\end{array}$ & 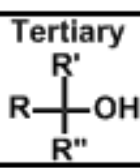 \\
\hline IMes & $R=2,4,6$-trimethylphenyl & $85 \%$ & $11 \%$ & $<1 \%$ \\
\hline IMe & $R=$ methyl & $79 \%$ & $72 \%$ & $<1 \%$ \\
\hline
\end{tabular}

Grasa et al. presented a study comparing the reactivity of various organocatalytic compounds with n-heterocyclic carbenes of different functionalities for the transesterification of benzyl alcohol with methyl acetate [24]. Besides 2,6-di-isopropylphenyl functionalized n-heterocyclic carbenes (IPr) due to steric hindrance, all nheterocyclic catalysts tested significantly outperformed the conventional basic organocatalysts dimethylalkylpyridine (DMAP), 1,4-diazabicyclo[2.2.0]octane (DABCO), and 1,8-diazabicycloundec-7-ene (DBU) in the transesterification of primary alcohols, as shown in Table 4. 
Table 4. Performance of different organocatalysts on the transesterification of methyl acetate with benzyl alcohol [24].

\begin{tabular}{|c|c|c|c|c|c|}
\hline Abbr. & Organocatalyst & Yield & Abbr. & Organocatalyst & Yield \\
\hline Imes & & $93 \%$ & ICy & & $100 \%$ \\
\hline $\mathrm{IPr}$ & & $45 \%$ & DMAP & & $15 \%$ \\
\hline $\mathrm{I}^{\mathrm{t}} \mathrm{Bu}$ & & $100 \%$ & DABCO & & $45 \%$ \\
\hline IAd & & $100 \%$ & DBU & & $24 \%$ \\
\hline
\end{tabular}

The gradual metamorphosis from utilizing pyridine-based organocatalysts to imidazolidone-based organocatalysts has influenced the strategy used in designing new organocatalysts. By understanding the effects of basicity on catalytic activity, researchers have successfully developed a new set of enhanced catalysts for transesterification reactions. The next section focuses on another innovative design on catalyst structure to enhance the activity compared to their preceding counterparts.

\subsubsection{Bifunctional Organocatalysts}

The evolution of organocatalysts has led to dramatic improvements in reaction performances, as illustrated when comparing n-heterocyclic carbenes with DMAP. Chemists are continuously exploring new strategic designs to improve the performance of organocatalysts. Bifunctionality in organocatalysts offers an innovative method to improve catalytic activity through the assistance of a secondary functionality. In the case 
of nucleophilic organocatalysts, the incorporation of hydrogen bonding may increase the stability of the catalytic complexes formed, thus improving the execution of the acylation reaction.

Some of the more regularly used bifunctional catalysts are the thiourea-based organocatalysts. There are wide variations in the structure of thiourea bifunctional catalysts serving multiple purposes, but 1-(3,5-bis(trifluoromethyl)phenyl)-3-(2(dimethylamino)cyclohexyl)thiourea (BDCT), depicted in Figure 11, is one of the more widely used thiourea catalysts. The effectiveness of BDCT results from the bidente (double) hydrogen bonding allowing the substrate to form a more stable complex for the reaction. Inokuma et al. have demonstrated the hydrogen bonding effect of BDCT for the Michael-addition of methylene compounds [25]. Pratt et al. further demonstrated the versatility of BDCT for the nucleophilic ring opening attack lactides with additional support from the electron pair on the tertiary amine [26]. The success of bifunctional acid catalysts has led to the investigation of implementing bifunctionality in existing organocatalysts to improve their performance.

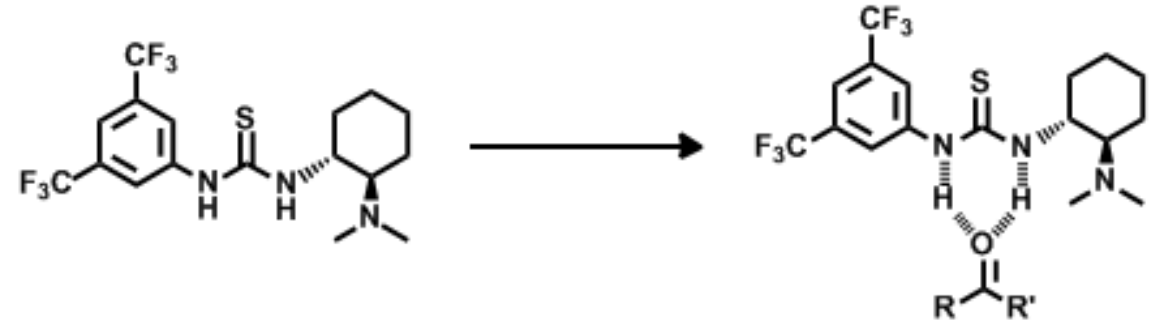

Figure 11. Illustration of BDCT and bidente hydrogen bonding formed due to bifunctionality [25]. 


\subsubsection{Amidine-based and Guanidine-based Organocatalysts}

Amidine based organocatalysts offer a new range of structures that could be applied to nucleophilic catalysis. The structure of amidine again allows it to become a hydrogen-bonding catalyst with limited nucleophilic catalytic activity due to the functionality of the amine groups. Existing organocatalysts using an amidine backbone include 1,5-diazabicyclo[4.3.0]non-5-ene (DBN) and 1,8-diazabicycloundec-7-ene (DBU), as illustrated in Figure 12 [27]. Amidine-based organocatalysts are generally less reactive compared to n-heterocyclic carbenes (Table 4), but are effective nucleophilic catalysts for specialized reactions such as the acylation of Baylis-Hillman adducts or catalysts for ring-opening polymerization [24,28-29].

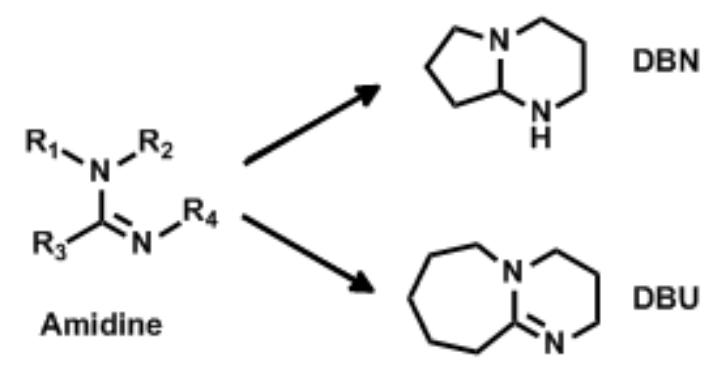

Figure 12. Illustration of amidine-derived organocatalysts.

In addition to amidine, guanidine-derived organocatalysts have also been developed for nucleophilic catalysis. The powerful reactivity of $1,5,7-$ triazabicyclo[4.4.0]dec-5-ene (TBD) has made it one of the most popular organocatalysts used for chemical synthesis. What makes TBD more effective is its bifunctionality resulting from the hydrogen bonding site and nucleophilic site, as shown in Figure 13 [30]. Green successfully demonstrated the transesterification of carboxylic and carbonic esters using TBD with relatively high conversions $[31,32]$. Furthermore, Schuchardt $e t$ 
al. compared the reactivity of TBD with other organocatalysts for the transesterification of vegetable oils [33]. The results revealed a $30 \%$ improvement in yield over the next best catalyst, triamino(imino)phosphoranes (BEMP), which is used in the hydrolysis of vegetable oils, and a $60 \%$ improvement in yield over DBU. The high basicity of TBD compared to DBU (150:3.4) explains the enhanced reactivity of TBD compared to other organocatalysts, while the tactically placed nucleophilic site on TBD explains the enhancement over BEMP.

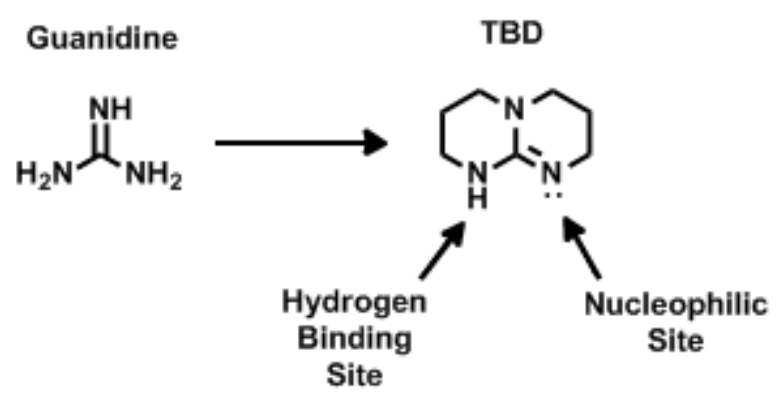

Figure 13. An illustration of guanidine-based organocatalyst TBD.

Similar to transesterification reactions, TBD has also demonstrated potential in ring-opening polymerizations using a nucleophilic attack on the carboxylic esters of $\mathrm{t}$ lactides [29,34]. This chapter focuses on the use of organocatalysts for ring-opening polymerizations. Slightly different from the focus of using organocatalysts for transesterification reactions, ring-opening polymerizations provide a mechanism that can be compared to the degradation of polymers using TBD. Chuma et al. investigated two possible catalytic pathways associated with the ring-opening of t-lactides: a hydrogenbonded mechanism and a covalently bounded or nucleophilic mechanism, illustrated in Figure 14 [30]. Based on computational chemistry models, Chuma et al. reported that the 
hydrogen-bonding pathway seemed most probable for the ring-opening polymerization due to large increases in the energy barrier height of the rate determine step (13.3 $\mathrm{kcal} / \mathrm{mol}$ ) compared to that of the covalent-bonding pathway $(23.5 \mathrm{kcal} / \mathrm{mol})$. However, the complex formed through ring-opening polymerization was more structurally confined than the one used for the transesterification of two independent substrates. So in the case of transesterification reactions, both mechanisms must be reanalyzed to determine the true catalytic pathway of TBD in the reaction.

In addition to the transesterification reactions, TBD has also been shown to perform aminolysis reactions with carboxylic esters, as demonstrated by Sabot et al. [35]. With the addition of TBD, the performance of the reactions resulted in amides with high yields (most $>90 \%$ ). These reactions were also performed under solvent free conditions, designating this as a "green" chemical process. This has dramatic implications for current industrial synthetic practices. Spychaj et al. demonstrated the ability to decompose polyethylene terephthalate (PET) using aminolysis reactions to form new aromatic amide diamine compounds (Figure 15) [36]. However, the assembled product consisted of non-symmetric constituents at relatively high conditions $\left(210^{\circ} \mathrm{C}\right)$. By combining the PET degradation reaction with $\mathrm{TBD}$, an environmentally benign process combining the high reactivity of the organocatalyst with the use of renewable reagents to form rigidly structured amides can be developed for future applications. 


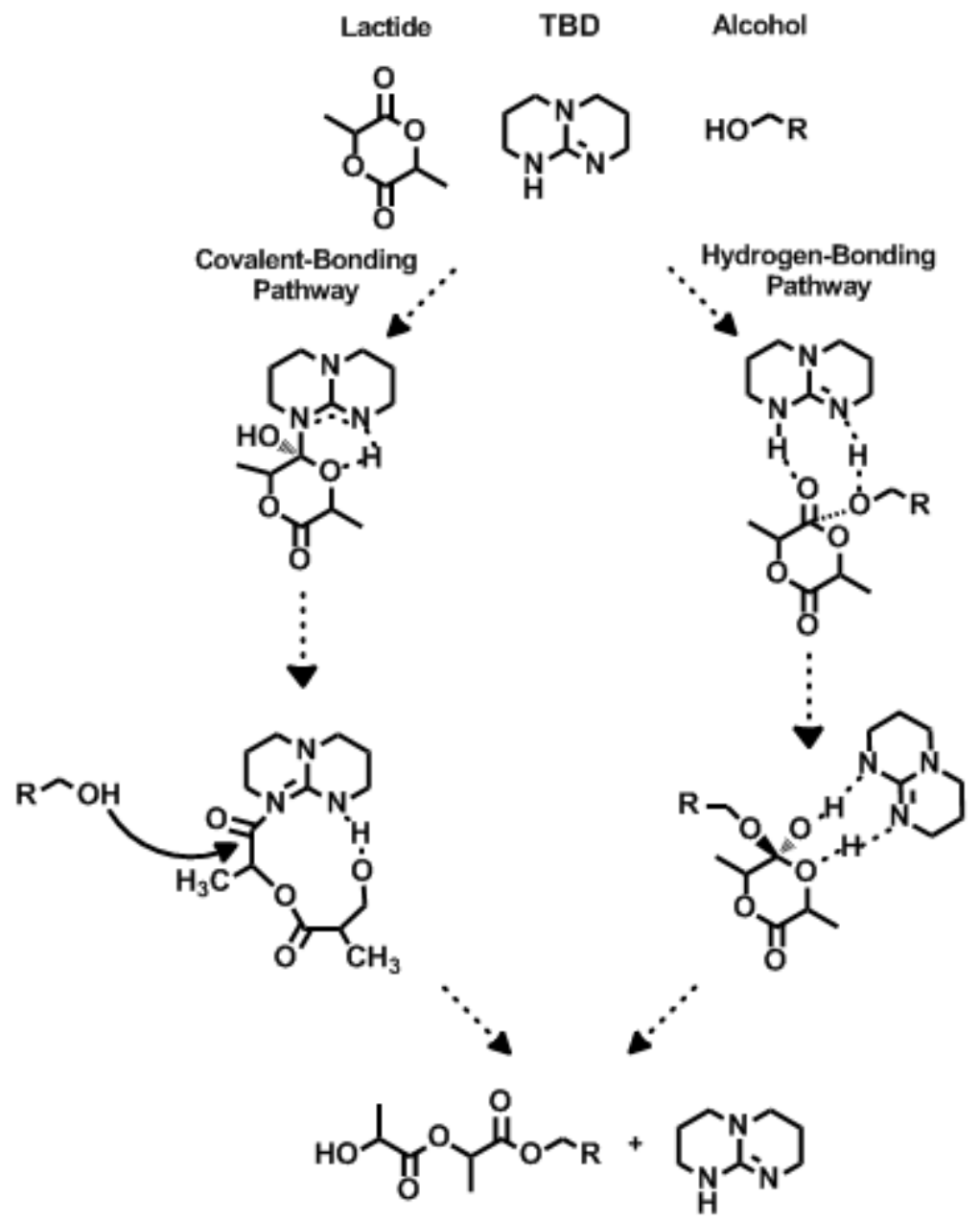

Figure 14. Catalytic mechanisms for the ring-opening polymerization of t-lactide using TBD [30].

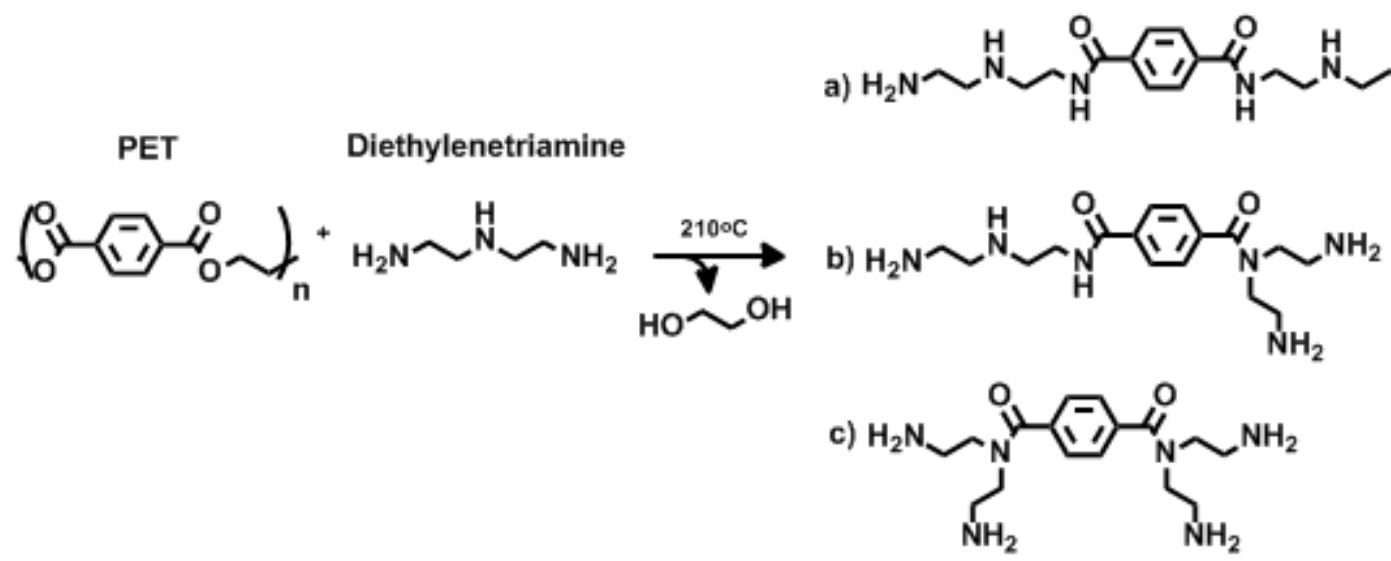

Figure 15. Aminolysis of polyethylene terephthalate with diethylenetriamine [36]. 
Structurally, TBD combines the enhanced basicity and nucleophilicity from pyridine-based and imidazolidone-based catalysts with the bifunctionality of the thiourea catalysts. By understanding the evolution of organocatalysts, one can comprehend the rationale behind the robust performance of TBD and its potential application to reform current chemical processes. Specifically, the degradation of PET into conventional or innovative monomers through transesterification/aminolysis reactions using TBD may provide a novel approach for averting the accumulation of plastic waste into our landfills while upholding the requirements that characterize this as a green chemistry process.

\subsection{Applications of Aromatic Amide/Ester Compounds}

An extensive library of potential monomers with special functional groups can be developed through transesterification/aminolysis of PET. For example, Spychaj et al. developed various derivatives of PET-Diethylenetriamine (PET-DETA) through the degradation of PET [36]. The versatility of TBD enables a large variety of rigid compounds to be formed. The versatility of TBD to produce an extensive library of potential monomers underscores the need for prospective applications for these model compounds. Although there are a variety of potential applications that could use aromatic amides, the focus of this project is the use of amides for the epoxy hardening process. 


\subsubsection{Epoxy Hardening}

Epoxies have been an integral component for various coating applications involving paints, adhesives, composites, and even insulating materials. Epoxies generally consist of two or more copolymers that form cross-linking polymers when thermally activated or cured. Gillham's time-temperature transformation (TTT) diagram, shown in Figure 16, illustrates the possible phases that thermosetting plastics can reach before they fully cross-link [37]. The goal is to reach the fully cured glass transition temperature $\left(\mathrm{Tg}_{\infty}\right)$ to ensure that the epoxy has polymerized completely without the drawbacks from mass transfer limitations that occur between the gelation and vitrification boundaries.

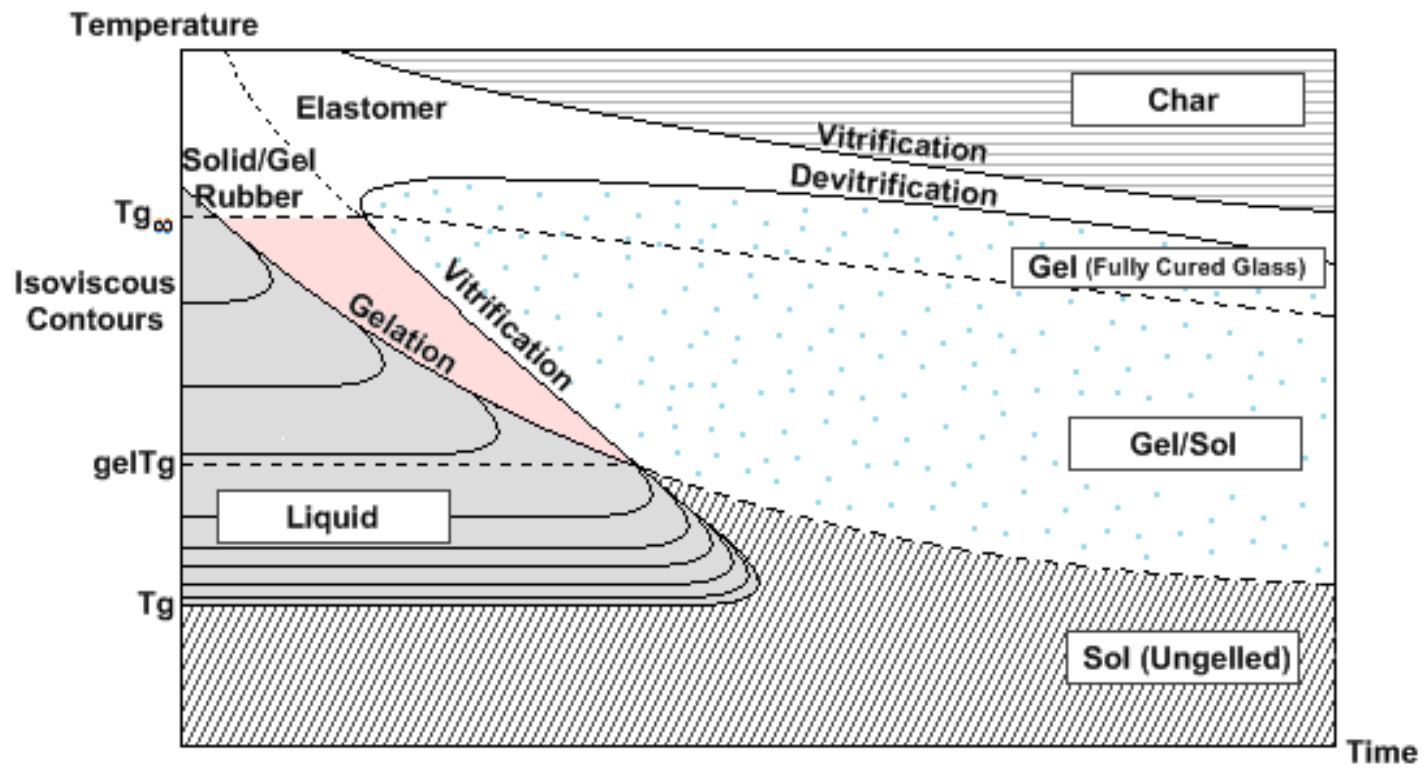

Figure 16. Time-temperature transformation profile for thermosetting plastics [37]. 
The increase in temperature allows the molecules to move around more freely throughout the volume since the viscosity is a function of temperature. The WilliamsLandel-Ferry model, shown in Equation 1, links the temperature with the viscosity, empirically demonstrating an exponential decline in viscosity at higher temperatures for thermosetting polymers [38].

$$
\ln \left(a_{T}\right)=\frac{-C_{1}\left(T-T_{r}\right)}{C_{2}+\left(T-T_{r}\right)}
$$

Equation 1

where $\mathrm{C}_{1}$ and $\mathrm{C}_{2}$ are empirically determined constants, $\mathrm{a}_{\mathrm{T}}$ is the shift factor, $\mathrm{T}$ is the desired operating temperature, and $\mathrm{T}_{\mathrm{r}}$ is the reference temperature.

Several different epoxy resins are used for epoxy hardening applications; however, the most commonly commercially used resin is bisphenol A diglycidyl ether (BADGE), shown in Figure 17 [39]. When near amine groups, the epoxide group is highly reactive and will form a covalent bond with each available hydrogen site. Riccardi et al. investigated the curing kinetics of BADGE with ethylenediamine (ED) to find that both an autocatalytic (reagents are the catalyst) and a second-order noncatalytic mechanism are present in the curing of epoxies [40]. Based on rate data, the $\operatorname{Tg}_{\infty}$ was estimated to be approximately $80^{\circ} \mathrm{C}$ for curing BADGE with ED at $60^{\circ} \mathrm{C}$ through the use of the differential scanning calorimeter (DSC). 


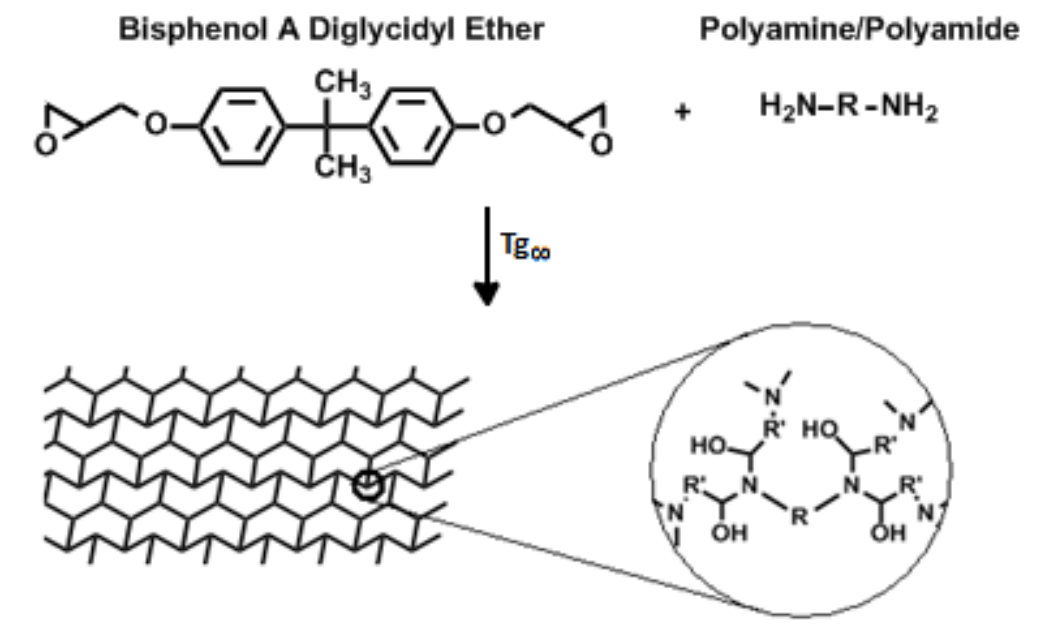

Figure 17. Cross-linking polymerization through epoxy hardening using BADGE and various polyamine/polyamides.

Aromatic polyamides provide sterner mechanical properties for compounds as a result of the combination between the pi interactions of the aromatic rings along with the improved hydrogen bonding from the presence of the carbonyl group. McLean et al. developed a process implementing aromatic amides as fortifiers to strengthen the hardening of epoxy resin [41]. The presence of the aromatic amides yielded tensile strength improvement of 7000 psi when incorporating acetoacetanilide (accompanied by phenylglycidylether) into an epoxy solution comprised of BADGE and methylene aniline (Figure 18) due to the additional hydrogen bonding occurring between the distributed amide groups. Iijima et al. further demonstrated the addition of terephthalate based monomers as modifiers for the epoxy curing between DDS and BADGE [42]. The addition of modifiers improved tensile strength from 135MPa at the control (ranging from $129 \mathrm{MPa}$ to $179 \mathrm{MPa}$ ) with a wide diversity in modulus from $3.44 \mathrm{GPa}$ at the control (ranging from $2.48 \mathrm{GPa}$ to $4.28 \mathrm{GPa}$ ). Spychaj et al. implemented an aromatic amide with PET degradation products directly as a curing agent for epoxy hardening, resulting in 
coating compounds with higher modulus of elasticity (108MPa to $3438 \mathrm{MPa}$ ) but a lower tensile strength (59MPa to $39 \mathrm{MPa}$ ) compared to the non-PET modified amines at $90^{\circ} \mathrm{C}$ curing temperatures for four hours [36]. The lower tensile strength and higher elasticity were perhaps the result of lower cross-linking density due to the gap formed by the aromatic amide groups. The modified characteristics from PET-derived amides provide solid characteristics for coating and adhesive applications.

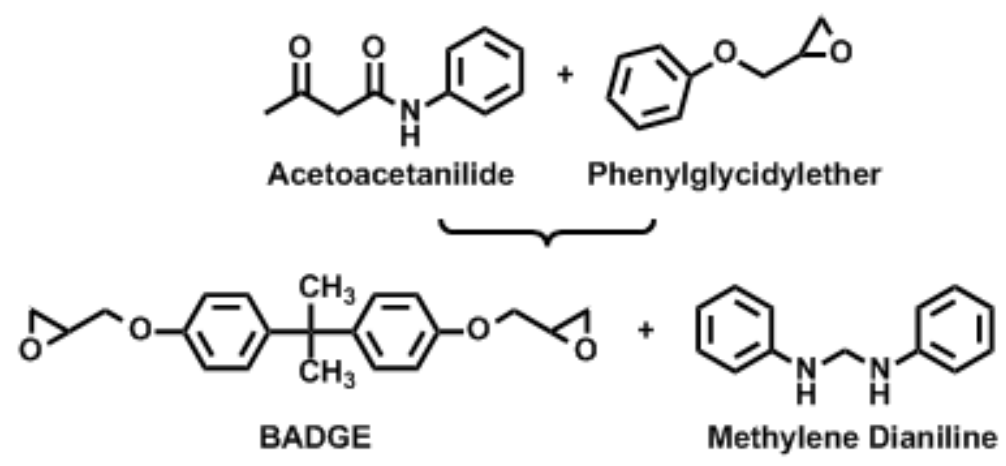

Figure 18. Implementing an aromatic amide as a fortifier for the epoxy hardening using methylene dianiline as the copolymer.

Aromatic amides also present the difficulty of solubility within the epoxies. One way to combat this challenge is by dissolving the amides in polar solvents. The involvement of solvents in the epoxy hardening process presents a concern over how the solvents may affect the interaction between the two copolymers. Hong and Wu presented a study on the effects of solvents on the DSC readings of epoxy systems [43]. The addition of solvents at $5 \%$ by weight exhibited considerable alterations in the glass transition temperatures. For the BADGE-dicyandiamide/2-methyl imidazole system, the absence of solvents provided a $\mathrm{Tg}_{\infty}$ at $121{ }^{\circ} \mathrm{C}$ compared to the addition of $5 \%$ wt. toluene where the $\mathrm{Tg}_{\infty}$ was found to be $104^{\circ} \mathrm{C}$, each at a curing temperature at $150^{\circ} \mathrm{C}$ to $170^{\circ} \mathrm{C}$. 
Consequently, the effects from the inclusion of solvents in the epoxy systems must be considered when characterizing the properties of the epoxy complex.

\subsubsection{Interfacial Polymerization and Desalination Membranes}

As advances in polymer technology continue to grow, new methods are developed in order to encompass the diversity of polymers. Interfacial polymerization is one facet that is growing in popularity as a result of its high performance with a relatively simple process. Bradhury et al. presented a study mixing piperazine in an aqueous phase with the terephthaloyl chloride in the organic phase to form a polyamide at the interface with high molecular weight [44].

Interfacial polymerization has become a staple in developing thin film composites used for reverse osmosis membranes. One of the earliest studies done on polyamide based reverse osmosis membranes focused on monomeric aliphatic amines with terephthaloyl chloride, illustrated in Table 5 [45]. The membrane exhibited poor salt rejections with large water fluxes. 1,2-ethane formed weak polymers that broke when operated for reverse osmosis. Codette eventually established the use of trimesoyl chloride with piperazine as the highest performing components for polyamide reverse osmosis membrane with $\mathrm{MgSO} 4$ salt rejection at $97 \%$ while maintaining a high flux at 28 gfd (better than 1,2-ethanediamine with 1-6 gfd) [45]. Aliphatic diamines provided higher salt rejections, especially with sodium chloride salts, but at the cost of making them impractical for desalination. 
Table 5. Performance of reverse osmosis membranes with monomeric aliphatic diamines and terephthaloyl chloride [45].

\begin{tabular}{|c|c|c|c|}
\hline Aliphatic Diamine & Acyl Chloride & Water Flux (gfd) & Salt Rejection \% \\
\hline$\underbrace{\mathrm{H}_{2} \mathrm{~N}}_{\text {1,2-Ethanediamine }} \overbrace{}^{\mathrm{NH}_{2}}$ & & Brittle & Brittle \\
\hline$\underset{1,3 \text {-propanediamine }}{\mathrm{NH}_{2}}$ & Cl & 233 & 30.6 \\
\hline 1,6-hexanediamine & & 104 & 8.2 \\
\hline
\end{tabular}

Cadotte delved into the incorporation of aromatic amides on polysulfone membranes for reverse osmosis membrane synthesis over conventional aliphatic amines and piperazine $[45,46]$. The best performing aromatic amide involved the polymerization of m-phenylenediamine with trimesoyl chloride, which demonstrated high flux (>22 gfd) and high salt rejection $(>96 \%)$. The addition of aromatic compounds provided more vacancies throughout the membrane, reducing the restriction of the molecules to pass through the filter. Several studies have also been conducted by adding special functional groups in order to enhance the performance of polyamide membranes. Yong et al. implemented the use of diamines with sulfonic groups to improve permeability [47]. Through observation, increasing compositions of the diamine to sulfonic-functionalized diamines demonstrated increases in flux but decreases in salt rejection, requiring a precise balance to enhance the performance of the membrane. This example provides a compelling case for searching specialized diamines to improve reverse osmosis systems. 


\subsubsection{Applications in Specialty Polymers}

Polymers have become an integral part of various industries due to the wide application of their various properties. There is interest in finding stronger and more robust polymers to improve the durability of these applications. To improve the structural integrity of the polymer, it must be examined at the chemical level to see if any improvements in the chemical structure can be made. Sheth et al. presented studies on the effect of hydrogen bonding in segmented parts of polyurethane and polyurea copolymers with hard segments [48]. It is rather difficult for polymers with hard segments to form high molecular weight polymers due to their structurally inflexible properties. The incorporation of soft segments, as well as the addition of hydrogen bonding properties, allows the buildup of molecular weight while still maintaining the structural properties of the hard segments. Another study by Sheth et al. also demonstrated morphology of hard segmented polymers with 1,4-phenylene diisocyanate to form polyurethanes yielding high level storage modulus [49]. The understanding of hard-segmented copolymers is required for the application of PET-derived aromatic amides for elastomeric processing.

Click chemistry provides another means for developing highly rigid molecules potentially for polymers. Binder and Sachsenhofer provided a review of the various methods for metal catalyzed reaction between azide and alkyne functionalized compounds [50]. Feldman et al. also investigated the azide reaction with an aromatic halogen with various alkyne functionalized groups (Figure 19) [51]. Click chemistry 
may provide a viable method for forming rigid aromatic polymers with properly functionalized PET-derived compounds.

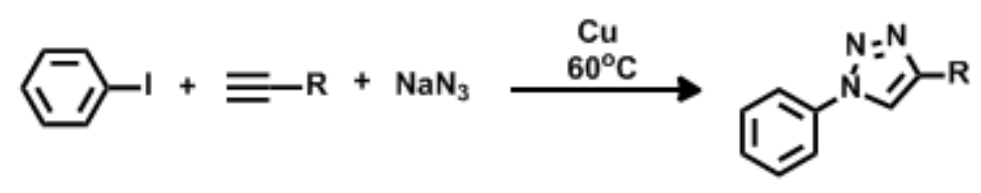

Figure 19. Click chemistry reaction illustration between an azide and an alkyl group [51].

\subsection{Economic Analysis}

One of the largest barriers in implementing the recycling of plastics lies in the cost differences of synthesizing plastics through chemical recycling versus oil derivatives. The main function of using organocatalysts is to reduce the operating conditions required during the chemical recycling of PET into its monomeric form. However, the cost of the organocatalysts plays an important role in the economics of chemical recycling. This section focuses on the economics of using organocatalysis to break PET down for resynthesis and degrading PET into high priced monomers for alternative applications. 


\subsubsection{Economics of PET Degradation Via Organocatalysis}

One of the benefits of using PET for packaging applications is the fact that the compounds required for PET synthesis are derived from highly abundant and cheap sources (oil and natural gas). An economic analysis was conducted comparing the current value of PET with the sum of the value of compounds required for organocatalytic recycling, derived from the prices shown in Table $6[52,53]$. However, several assumptions needed to be made to make the analysis more realistic. First, the amount of catalyst used consisted of only 5\% loading by weight for each gram of PET. Second, only the molar equivalence of ethylene glycol for 1 gram of PET was considered. This was because reagents can be separated out after purification and recycled for additional PET degradation reaction, as opposed to tossing excess reagents and using a fresh batch. Lastly, PET used for organocatalytic recycling was assumed to be taken from waste and assumed to have negligible value. By incorporating these assumptions into the calculation, the price of developing PET through oil was compared with the price of developing PET through organocatalytic decomposition of PET, as demonstrated in

\section{Table 7.}

Table 6. Current prices for PET and chemicals required for organocatalytic recycling.

\begin{tabular}{|l|c|}
\hline \multicolumn{1}{|c|}{ Compound } & Price $(\$ / g)$ \\
\hline Polyethylene Terephthalate (PET) & $\$ 1.51 / \mathrm{kg}$ \\
\hline 1,5,7-triazabicyclo[4.4.0]dec-5-ene (TBD) & $\$ 0.672 / \mathrm{g}^{*}$ \\
\hline Ethylene Glycol (EG) & $\$ 0.88 / \mathrm{kg}$ \\
\hline
\end{tabular}

*Estimated price calculated based on empirical formulation of privatized data 
Table 7. Economic analysis of synthesizing PET from oil and from organocatalytic recycling.

\begin{tabular}{|c|c|c|c|c|}
\hline PET Synthesis & Compound & Price (\$/g) & $\begin{array}{c}\text { Amount for } 1 \mathrm{~g} \\
\text { of PET }\end{array}$ & $\begin{array}{c}\text { Price for } 1 \mathrm{~g} \text { of } \\
\text { PET }\end{array}$ \\
\hline $\begin{array}{c}\text { From Oil } \\
\text { Derivatives }\end{array}$ & $\begin{array}{l}\text { PET } \\
\text { Total }\end{array}$ & $\$ 0.00151 / \mathrm{g}$ & $1 \mathrm{~g}$ & $\begin{array}{l}\$ 0.00151 \\
\$ \mathbf{0 . 0 0 1 5 1}\end{array}$ \\
\hline $\begin{array}{c}\text { From } \\
\text { Organocatalytic } \\
\text { Recycling }\end{array}$ & $\begin{array}{c}\text { PET } \\
\text { EG } \\
\text { TBD } \\
\text { Total }\end{array}$ & $\begin{array}{c}- \\
\$ 0.00088 / \mathrm{g} \\
\$ 0.672 / \mathrm{g}\end{array}$ & $\begin{array}{c}1 \mathrm{~g} \\
0.646 \mathrm{~g} \\
0.05 \mathrm{~g}\end{array}$ & $\begin{array}{c}- \\
\$ 0.000546 \\
\$ 0.0336 \\
\$ \mathbf{0 . 0 3 4 1 6}\end{array}$ \\
\hline
\end{tabular}

There is a significant price discrepancy between developing PET straight from oil derivatives and from organocatalytic recycling. The expense stems primarily from the high price of TBD (nearly 98\%). This analysis demonstrates the difficulty of using chemical recycling, especially with organocatalysts, to synthesize PET. However, there are certain possibilities that may make this feasible in the future. First, the cost to synthesize PET from oil derivatives is directly linked to the price of oil. Over the last few decades, oil prices have spiked due to decreased reserves. If the price of oil continues to rise over the next few decades, synthesizing PET using oil derivatives may become less economical than chemical recycling. In addition, TBD has only been produced in lab scale quantities, as it has only been discovered within the last five years. Large-scale production of TBD may emerge as a result of potential new applications for organocatalysts. Consequently, the price of TBD will drop tremendously due to the benefits gained from the economies of scale. The combination of these two factors may eventually allow for the mainstream use of organocatalysis for the chemical recycling of 
PET. Meanwhile, the next section presents an alternative for modifying PET in order to develop a more economically viable process for the near future.

\subsubsection{Economics of Incorporating PET-derived Diamines for Epoxy Hardening}

As discussed in the previous section, chemical recycling of PET using organocatalysis may not be a cost-effective method for synthesizing PET. Alternatively, PET may undergo aminolysis, which is discussed in Chapter 3, to form bifunctional aromatic amides that may have potential applications as modifiers for epoxy hardening. However, it is important to determine whether the process is financially competitive with common epoxy modifiers. The same assumptions (5\% weight catalyst loading of TBD, molar equivalence of PET used, and PET reagent is valueless) made for the economic analysis for the organocatalytic recycling of PET was applied for this process as well. However, the amounts required were based on a $10 \mathrm{~g}$ scale of bisphenol A propoxylate diglycidyl ether (BAPDGE). Furthermore, only 15\% of the molar equivalence of BAPDGE was used for the calculation in order to demonstrate the compound more as a modifier than a full-fledged curing agent. Using these assumptions, we can calculate the cost based on how much of the PET-derived diamines are required for the process and, in turn, how much of the starting material is needed to make the PET compounds.

The results for the economic analysis for using PET-derived diamine products as epoxy modifiers are summarized in Table 8 . In this case, the price of a typically used modifier/curing agent, triethylenetetramine, was compared to the price of the starting material to make the equivalence for the PET-derived diamines. For 15\% equivalence of 
the modifier to a $10 \mathrm{~g}$ sample of BAPDGE, the calculated cost for triethylenetetramine was $\$ 4.96 / \mathrm{kg}[54]$. For the same ratios, the cost of the starting materials for PETethylenediamine and PET-m-Xylylenediamine were $\$ 7.5 / \mathrm{kg}$ and $\$ 20.96 / \mathrm{kg}$, respectively $[55,56]$. The prices for developing PET-derived diamines were within one order of magnitude, slightly better than the prices of organocatalytic recycling of PET for PET synthesis. In addition, the properties of the aromatic amides might enhance the properties of the epoxy polymer, allowing the price discrepancy to be reasonable.

Table 8. Economic analysis of implementing PET-decomposed polyamides for epoxy hardening applications.

\begin{tabular}{|c|c|c|c|c|}
\hline Modifier & Reagent & $\begin{array}{c}\text { Reagent Price } \\
(\$ / g)\end{array}$ & $\begin{array}{l}\text { Amount } \\
\text { Required }\end{array}$ & $\begin{array}{c}\text { Price For } \\
\text { Amount }\end{array}$ \\
\hline Triethylenetetramine & $\begin{array}{l}\text { TETA } \\
\text { Total }\end{array}$ & $\$ 0.031 / \mathrm{g}$ & $0.01601 \mathrm{~g}$ & $\begin{array}{l}\$ 0.00496 \\
\$ \mathbf{0 . 0 0 4 9 6}\end{array}$ \\
\hline $\begin{array}{l}\text { PET-Ethylenediamine } \\
\text { (PET-ED) }\end{array}$ & $\begin{array}{c}\text { ED } \\
\text { TBD } \\
\text { PET } \\
\text { Total }\end{array}$ & $\begin{array}{c}\$ 0.00364 / \mathrm{g} \\
\$ 0.672 / \mathrm{g} \\
-\end{array}$ & $\begin{array}{l}0.1316 \mathrm{~g} \\
0.0105 \mathrm{~g} \\
0.2104 \mathrm{~g}\end{array}$ & $\begin{array}{c}\$ 0.000479 \\
\$ 0.007071 \\
- \\
\$ \mathbf{0 . 0 0 7 5}\end{array}$ \\
\hline $\begin{array}{l}\text { PET-m-Xylylenediamine } \\
\text { (PET-mX) }\end{array}$ & $\begin{array}{c}\mathrm{mX} \\
\mathrm{TBD} \\
\mathrm{PET} \\
\text { Total } \\
\end{array}$ & $\begin{array}{c}\$ 0.038 / \mathrm{g} \\
\$ 0.672 / \mathrm{g} \\
-\end{array}$ & $\begin{array}{l}0.4798 \mathrm{~g} \\
0.0169 \mathrm{~g} \\
0.3385 \mathrm{~g}\end{array}$ & $\begin{array}{c}\$ 0.0182 \\
\$ 0.001137 \\
- \\
\$ \mathbf{0 . 0 2 9 6} \\
\end{array}$ \\
\hline
\end{tabular}

\subsection{Literature Review Summary}

The development of organocatalysts has facilitated "green" reform of the current chemical process. In addition, chemically degrading PET waste using organocatalysts, in this case TBD, into either its basic constituents or into new novel monomers, provides a 
valuable solution to the global spanning problem. Through analyzing the bifunctional structure of TBD, one can understand its potency for the chemical degradation of PET into a diverse set of products, specifically aromatic amides. However, it is important to determine how these aromatic amides that are formed from PET degradation can be incorporated into practical applications. These practical applications, such as epoxy hardening, provide not only a novel method for using PET waste, but economic incentives as well. For this project, the focus was on using the products from PET degradation to develop modifiers for epoxy hardening. 


\section{CHAPTER THREE}

\section{PROJECT OBJECTIVES}

The main objective of this project is to develop a library of polyethylene terephthalate (PET)-derived amines and characterize them using nuclear magnetic resonance (NMR) spectroscopy. The degradation of PET was carried out in solvent-free conditions using the organocatalyst 1,5,7-triazabicyclo[4.4.0]dec-5-ene (TBD). By using a diverse set of amines, a library of polyamides was formed through aminolysis reactions using degraded PET as the structural basis. The resulting set of amines included primary amines (aliphatic, aromatic, click functionalized, tertiary functionalized), secondary amines, and asymmetric amines.

In addition to forming new rigid monomers using chemically recycled PET waste, a secondary objective of the project was to determine a commercially viable process to incorporate the PET-derived monomers. This project focused solely on using PETderived diamines as modifiers for epoxy hardening. Four PET-derived diamines (PETEthylenediamine, PET-m-Xylylenediamine, PET-Diamino-N-methyldipropylamine, and PET-Diethylenediamine) were used for the epoxy hardening studies. The effect of PETderived diamines on the strengthening of epoxy polymers in comparison to their basic counterparts was analyzed using a solvent-based procedure in order to dissolve the diamines into the epoxy resin for thermal analysis. Differential scanning calorimetry and dynamic mechanical analysis were used to analyze curing behavior of the epoxy 
composites with the PET-derived diamines. The thermal characterization of the epoxy composites not only demonstrated the ability of the PET-derived diamines to bind with the epoxy resin, but also illustrated the effects of the PET-diamine structure on the curing behavior. The following chapter focuses on the process of developing the PET degraded monomers and the preparation and characterization of the epoxy polymers. 


\section{CHAPTER FOUR}

\section{MATERIALS AND METHODS}

The successful application of organocatalysts for aminolysis reactions discussed in Chapter 2 suggests the incorporation of organocatalysts for the chemical recycling of polyethylene terephthalate. The focus of the first section of this chapter is the procedure and materials used for the degradation of PET into novel compounds. The next section of the chapter focuses on the epoxy hardening process and the implementation of the thermal characterization techniques used to describe the composite thermal properties resulting from the PET-derived compounds.

\subsection{Organocatalytic Decomposition of Polyethylene Terephthalate}

The synthesized compounds formed from degraded PET vary based on the reagents used for the aminolysis reactions. This allows the development of a wide variety of compounds based on the structure and functionality of the reagent compounds.

The reagents tested were divided into subgroups based on similarities in structures (Table 9). Due to the diverse properties of each reagent, each process required slightly different conditions to operate and purify depending on the compound. This section first discusses the materials used for the decomposition of PET and the general procedure for synthesizing the PET-derived monomers. This section further discusses the procedure for reusing the reagents to develop a more efficient process. 
Table 9. Potential reagents for PET decomposition and modification.

\begin{tabular}{|c|c|c|c|c|}
\hline$\#$ & Subgroup & Reagent Name & Nickname & Chemical Structure \\
\hline 1 & \multirow[t]{3}{*}{ Aliphatic } & Ethylenediamine & ED & $\mathrm{H}_{2} \mathrm{~N} \widetilde{ }$ \\
\hline 2 & & 1,4 Diaminobutane & $\mathrm{DAB}$ & $\mathrm{H}_{2} \mathrm{~N}^{-}$ \\
\hline 3 & & 1,6 Diaminohexane & DAH & 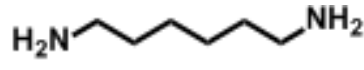 \\
\hline 4 & \multirow[t]{4}{*}{ Aromatic } & $\mathrm{m}$-Xylylenediamine & $\mathrm{mX}$ & \\
\hline 5 & & p-Xylylenediamine & $\mathrm{pX}$ & \\
\hline 6 & & p-Aminobenzylamine & $\mathrm{ABA}$ & \\
\hline 7 & & p-phenylenediamine & PPD & \\
\hline 8 & \multirow[t]{2}{*}{ Secondary } & Piperidine & PPI & \\
\hline 9 & & Piperazine & PPA & \\
\hline 10 & \multirow[t]{3}{*}{$\begin{array}{c}\text { Non- } \\
\text { symmetric } \\
\text { (Selective) }\end{array}$} & Ethanolamine & EthA & \\
\hline 11 & & $\begin{array}{c}\text { 1-(2- } \\
\text { Aminoethyl)piperazine }\end{array}$ & AEP & \\
\hline 12 & & Diethylenetriamine & DETA & $\mathrm{H}_{2} \mathrm{~N}$ \\
\hline 13 & \multirow[t]{2}{*}{ Click } & Allylamine & Allyl & \\
\hline 14 & & Propargylamine & Pro & \\
\hline 15 & \multirow[t]{3}{*}{$\begin{array}{c}\text { Tertiary } \\
\text { Functionality }\end{array}$} & $\begin{array}{c}\text { 3,3'-Diamino-N- } \\
\text { methyldipropylamine }\end{array}$ & DAMDPA & $\mathrm{H}_{2} \mathrm{~N} \sim$ \\
\hline 16 & & 1,6-Diaminopyridine & DAP & \\
\hline 17 & & Tris(2-aminoethyl)amine & TAEA & \\
\hline
\end{tabular}




\subsubsection{Materials}

The main set of materials included the chemicals used for PET degradation, purification, and analysis. PET was provided in the form of post-consumed plastic bottles. The plastic bottle was stripped of all labels and washed thoroughly with water to remove any potential contaminants. Then, the bottle was further prepared by cutting it into squares (flakes) no larger than $1 \mathrm{~cm}$ by $1 \mathrm{~cm}$. TBD and the reagents used for PET degradation (displayed in Table 9) were purchased from Sigma-Aldrich and provided by the IBM Almaden Research Facility. The compounds were of analytical grade and used straight from the bottle without additional purification. Solvents used for the purification steps were purchased from J.T. Baker and used straight from the bottle. Finally, deuterated dimethylsulfoxide, used for nuclear magnetic resonance spectroscopy characterization of the PET-derived monomers, was purchased from Cambridge Isotope Laboratories and used straight from the bottle.

The PET-derived products were characterized through proton nuclear magnetic resonance spectroscopy (HNMR). The basic idea of HNMR spectroscopy is that hydrogen, with an odd number of protons, has a spin that can affect the magnetic field provided by an instrument to give off resonance signals. The signals released are proportional to the number of protons, the influence of electronegative atoms on the protons, and the spin coupling effects formed from neighboring protons (J-coupling). The signals differ based on the functional groups of the tested sample, which provides a great tool for determining the chemical structure of the PET-derived monomers. The 
NMR instrument operated at IBM Almaden Research Center was the Avance 400 model purchased from Bruker Instruments.

\subsubsection{Experimental Procedure for PET Decomposition}

The experimental design for the chemical degradation of PET was based on the principles of green chemistry to develop a more environmentally friendly process. The reaction conditions focused primarily on solvent-free conditions, at temperatures less than $200^{\circ} \mathrm{C}$, and near atmospheric pressure or vacuum. Compared to the traditional chemical recycling processes described by Paszun et al., the conditions required for PET degradation using TBD made for a less demanding process that met green requirements [12]. This section focuses on the reasoning behind selecting the appropriate reaction conditions along with the apparatus for the experiment. This section also describes in detail the PET aminolysis procedure performed for each reagent listed in Table 9. The HNMR spectra of each successful product can be found in the Appendix.

The general procedure for the PET aminolysis reactions consisted of adding between 1-5 grams of shredded PET, 3-8 wt.\% of TBD (based on mass of PET), excess amounts of the respective reagent, and a magnetic stir bar into a $25 \mathrm{~mL}$ Schlenk tube. Then the tube was installed into the apparatus illustrated in Figure 20. Choosing the reaction temperature was rather complicated, as it depends on the properties of the reagent itself. The key point, as discussed earlier, is that the reagents must be melted to liquid form since the reaction volume is comprised mostly of the reagent. The general strategy for deciding the reaction temperatures followed the guidelines listed in Table 10. 
There were additional concerns over selecting temperatures. First, at temperatures above $150^{\circ} \mathrm{C}$, TBD sublimes out, forming a white crown above the reaction volume. As a result, it was imperative to scrape the TBD back into the reaction volume or add more TBD to continue the reaction. This project did not focus on reaction temperatures over $200^{\circ} \mathrm{C}$, as TBD becomes inactive at such high temperatures and a new bath medium would be required (i.e., sand instead of oil) to reach such temperatures. For nonsymmetric reagents, the goal was to target the most reactive side. Therefore, it was important not to bring the temperature too high so that the PET could attack both sides, providing a mixture of different PET compounds. Table 11 provides the list of reagents used, along with their selected reaction temperatures based on their boiling and melting points.

Table 10. General strategy for selecting temperature for the reaction.

\begin{tabular}{|c|c|}
\hline$\#$ & Temperature Condition of Reaction Volume $\left.\mathbf{~}_{\mathbf{R V}}\right)$ \\
\hline 1 & $\mathrm{~T}_{\mathrm{RV}} \geq$ Melting Point of Reagent \\
\hline 2 & $\mathrm{~T}_{\mathrm{RV}} \leq$ Boiling Point of Reagent \\
\hline 3 & $\mathrm{~T}_{\mathrm{RV}} \leq 200^{\circ} \mathrm{C}$ (Temperature Limits of TBD) \\
\hline 4 & $\mathrm{~T}_{\mathrm{RV}} \geq 40^{\circ} \mathrm{C}$ (Needed to Surpass Energy Barrier) \\
\hline 5 & Higher $\mathrm{T}_{\mathrm{RV}}$ for Less Reactive Reagents \\
\hline
\end{tabular}




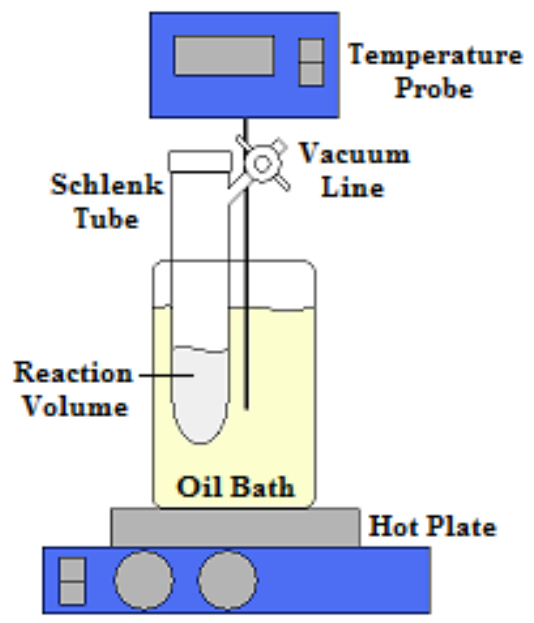

Figure 20. Illustration of the experimental apparatus set-up for PET-degradation reaction.

Once the temperature conditions were set, the Schlenk tube was inserted into the apparatus based on the set-up illustrated in Figure 20. Although it was not completely necessary, the reactions were held under vacuum as a safety precaution to prevent a buildup in pressure due to released ethylene glycol. As the PET degraded, the solution turned homogeneous (i.e., flakes disappeared). The time required for full conversion depends on the reactivity of the reagents. In the case regarding ethylenediamine as a reagent, with the reaction shown in Figure 21, the reaction volume should reach homogeneity within a few hours. However, all reactions were set overnight or over the weekend as a cautionary measure to provide enough time for large conversions.

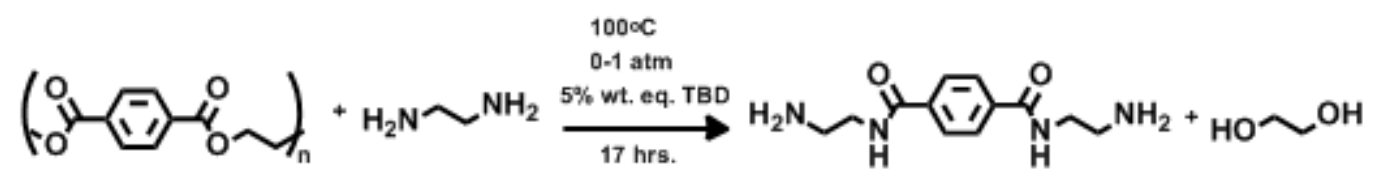

Figure 21. PET degradation reaction conditions using ethylenediamine reagent 
Table 11. List of reaction temperatures for various reagents in the decomposition of PET.

\begin{tabular}{|c|c|c|c|c|}
\hline$\#$ & Compound & Chemical Structure & $\begin{array}{c}\text { Boiling/Melting Point } \\
\text { of Reagent }\end{array}$ & $\begin{array}{c}\text { Reaction } \\
\text { Temperature }\end{array}$ \\
\hline 1 & ED & & $118^{\circ} \mathrm{C}(\mathrm{BP})$ & $100^{\circ} \mathrm{C}$ \\
\hline 2 & $\mathrm{DAB}$ & $\mathrm{H}_{2} \mathrm{~N}^{-}$ & $28^{\circ} \mathrm{C}(\mathrm{MP})$ & $100^{\circ} \mathrm{C}$ \\
\hline 3 & $\mathrm{DAH}$ & 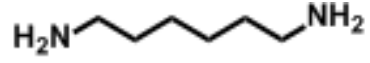 & $42^{\circ} \mathrm{C}(\mathrm{MP})$ & $100^{\circ} \mathrm{C}$ \\
\hline 4 & $\mathrm{mX}$ & & $265^{\circ} \mathrm{C} / 745 \mathrm{mmHg}(\mathrm{BP})$ & $100^{\circ} \mathrm{C}$ \\
\hline 5 & $\mathrm{pX}$ & & $63^{\circ} \mathrm{C}(\mathrm{MP})$ & $100^{\circ} \mathrm{C}$ \\
\hline 6 & $\mathrm{ABA}$ & & $37^{\circ} \mathrm{C}(\mathrm{MP})$ & $100^{\circ} \mathrm{C}$ \\
\hline 7 & PPD & & $143^{\circ} \mathrm{C}(\mathrm{MP})$ & $150^{\circ} \mathrm{C}$ \\
\hline 8 & PPI & & $106^{\circ} \mathrm{C}(\mathrm{BP})$ & $95^{\circ} \mathrm{C}$ \\
\hline 9 & PPA & & $\begin{array}{l}145^{\circ} \mathrm{C}(\mathrm{BP}) \\
109^{\circ} \mathrm{C}(\mathrm{MP})\end{array}$ & $135^{\circ} \mathrm{C}$ \\
\hline 10 & EthA & & $\begin{array}{l}11^{\circ} \mathrm{C}(\mathrm{MP}) \\
170^{\circ} \mathrm{C}(\mathrm{BP})\end{array}$ & $80^{\circ} \mathrm{C}$ \\
\hline 11 & AEP & & $218^{\circ} \mathrm{C}(\mathrm{BP})$ & $80^{\circ} \mathrm{C}$ \\
\hline 12 & DETA & & $199^{\circ} \mathrm{C}(\mathrm{BP})$ & $80^{\circ} \mathrm{C}$ \\
\hline 13 & Allyl & & $52^{\circ} \mathrm{C}(\mathrm{BP})$ & $47^{\circ} \mathrm{C}$ \\
\hline 14 & Pro & & $83^{\circ} \mathrm{C}(\mathrm{MP})$ & $67^{\circ} \mathrm{C}$ \\
\hline 15 & DAMDPA & $\sim \mathrm{NH}_{2}$ & $110^{\circ} \mathrm{C} / 6 \mathrm{mmHg}(\mathrm{BP})$ & $110^{\circ} \mathrm{C}$ \\
\hline 16 & DAP & & $122^{\circ} \mathrm{C}(\mathrm{MP})$ & $150^{\circ} \mathrm{C}$ \\
\hline 17 & TAEA & & $114^{\circ} \mathrm{C} / 15 \mathrm{mmHg}(\mathrm{BP})$ & $110^{\circ} \mathrm{C}$ \\
\hline
\end{tabular}

*All boiling point/melting point temperatures can be found on the Sigma-Aldrich website: http://www.sigmaaldrich.com 
The final step for synthesizing PET-derived compounds is purification.

Purification consisted of removing the crude product from the Schlenk tube into a semipolar organic solvent such as ethyl acetate. This exploits the fact that aromatic amides are generally insoluble in most organic solvents. Water could have been used as a separation solvent for a few of the products, but some PET-derived compounds may be partially soluble in the aqueous solution. Therefore, by using semi-polar solvents, the PET-derived compounds were easily separated out from the reagent and catalyst through a simple filtration process with frit funnel. The product was then analyzed through calculating the product yield and identifying the compound through HNMR spectroscopy. The next few sections present in detail the specifics on the reactions performed for each reagent.

\subsubsection{Synthesis of PET-derived Aliphatic Diamines}

$N^{l}, N^{4}$-bis(2-aminoethyl)terephthalamide (PETED)

Post consumed polyethylene terephthalate flakes (PET, $3.06 \mathrm{~g}, 16 \mathrm{mmol}$ ) were placed into a $25 \mathrm{~mL}$ Schlenk tube along with excess amounts of ethylenediamine (ED, 11.83g, $0.2 \mathrm{~mol}$ ) and 1,5,7-triazabicyclo[4.4.0]dec-5-ene (TBD, $0.18 \mathrm{~g}, 1.3 \mathrm{mmol})$. The tube was then heated under vacuum at $100^{\circ} \mathrm{C}$ overnight $(17 \mathrm{~h})$. The homogeneous solution was then dumped in $250 \mathrm{~mL}$ of ethyl acetate and filtered. The resulting white powder was then dried in a vacuum oven at $50^{\circ} \mathrm{C}$, yielding the desired product (PETED) with high purity (3.95 g, 99\%). 
$N^{l}, N^{4}$-bis(4-aminobutyl)terephthalamide (PETDAB)

PET flakes (1.02 g, $5.3 \mathrm{mmol})$, 1,4-diaminobutane (DAB, $6.49 \mathrm{~g}, 73 \mathrm{mmol})$ and 1,5,7-triazabicyclo[4.4.0]dec-5-ene (TBD, $0.07 \mathrm{~g}, 0.5 \mathrm{mmol}$ ) were placed in the Schlenk tube and then heated under vacuum at $100^{\circ} \mathrm{C}$ over the weekend $(50 \mathrm{~h})$. Purification of the product was performed using the procedure discussed in the previous section, with PETED yielding the desired product (PETDAB) in the form of a white powder with high purity $(1.37 \mathrm{~g}, 85 \%)$.

$N^{l}, N^{4}$-bis(6-aminohexyl)terephthalamide (PETDAH)

PET flakes (2.98 g, $15.5 \mathrm{mmol}), 1,6$-diaminohexane (DAH, $11.47 \mathrm{~g}, 1 \mathrm{~mol})$ and 1,5,7-triazabicyclo[4.4.0]dec-5-ene (TBD, $0.12 \mathrm{~g}, 0.9 \mathrm{mmol}$ ) were placed in the Schlenk tube and then heated under vacuum at $100^{\circ} \mathrm{C}$ overnight $(18 \mathrm{~h})$. Purification of the product was performed using the procedure discussed in the previous section with PETED yielding the desired product (PETDAH) in the form of a white powder with high purity (5.24 g, 93\%).

4.1.2.2 Synthesis of PET-Based Monomers with Phenyl Containing Diamines $N^{l}, N^{4}$-bis(3-(aminomethyl)benzyl)terephthalamide (PETmX)

PET flakes (5.02 g, $26.1 \mathrm{mmol})$, m-xylylenediamine (mX, $16.97 \mathrm{~g}, 1.2 \mathrm{~mol})$ and 1,5,7-triazabicyclo[4.4.0]dec-5-ene (TBD, $0.17 \mathrm{~g}, 1.2 \mathrm{mmol}$ ) were placed in the Schlenk tube and then heated under vacuum at $100^{\circ} \mathrm{C}$ overnight $(17 \mathrm{~h})$. The homogeneous solution was then dumped in $250 \mathrm{~mL}$ of ethyl acetate and filtered. The resulting white 
powder was then dried in a vacuum oven at $50^{\circ} \mathrm{C}$, yielding the desired product (PETmX) with high purity $(7.59 \mathrm{~g}, 72 \%)$.

\section{$N^{1}, N^{4}$-bis(4-(aminomethyl)benzyl)terephthalamide (PETpX)}

PET flakes (1.01 g, $5.23 \mathrm{mmol}$ ), p-xylylenediamine (pX, $3.58 \mathrm{~g}, 26 \mathrm{mmol})$, and 1,5,7-triazabicyclo[4.4.0]dec-5-ene (TBD, $0.11 \mathrm{~g}, 0.8 \mathrm{mmol}$ ) were placed in the Schlenk tube and then heated under vacuum at $100^{\circ} \mathrm{C}$ over the weekend $(50 \mathrm{~h})$. The homogeneous solution was then dumped in $250 \mathrm{~mL}$ of ethyl acetate and filtered. The resulting white powder was then dried in a vacuum oven at $50^{\circ} \mathrm{C}$, yielding the desired product (PETpX) with high purity $(1.59 \mathrm{~g}, 75 \%)$.

\section{$N^{1}, N^{4}$-bis(4-aminobenzyl)terephthalamide (PETABA)}

PET flakes (2.90 g, $15 \mathrm{mmol}$ ), 4-aminobenzylamine (ABA, $9.59 \mathrm{~g}, 78 \mathrm{mmol}$ ), and 1,5,7-triazabicyclo[4.4.0]dec-5-ene (TBD, $0.17 \mathrm{~g}, 1.2 \mathrm{mmol}$ ) were placed in the Schlenk tube and then heated under vacuum at $100^{\circ} \mathrm{C}$ over the weekend $(50 \mathrm{~h})$. The homogeneous solution was then dumped in $250 \mathrm{~mL}$ of ethyl acetate and filtered. The resulting yellow powder was then dried in a vacuum oven at $50^{\circ} \mathrm{C}$, yielding the desired product (PETABA) with high purity $(4.86 \mathrm{~g}, 85 \%)$.

$N^{1}, N^{4}$-bis(4-aminophenyl)terephthalamide (PETpPD)

PET flakes (4.01 g, $21 \mathrm{mmol}$ ), p-phenylenediamine (pPD, $17.25 \mathrm{~g}, 1.6 \mathrm{~mol}$ ), and 1,5,7-triazabicyclo[4.4.0]dec-5-ene (TBD, $0.2 \mathrm{~g}, 1.5 \mathrm{mmol})$ were placed in the Schlenk tube and then heated under vacuum at $150^{\circ} \mathrm{C}$ overnight $(20 \mathrm{~h})$. The homogeneous solution was then dumped in $250 \mathrm{~mL}$ of ethyl acetate and filtered. However, H-NMR 
data showed significant amounts of the reagent within the product. The product was dissolved in $10 \mathrm{~mL}$ of methanol and precipitated out in $250 \mathrm{~mL}$ of ethyl acetate. The resulting blue powder was then dried in a vacuum oven at $50^{\circ} \mathrm{C}$ yielding the product (PETpPD) with significant amounts of the reagent remaining in the product $(7.77 \mathrm{~g}$, $>100 \%)$.

\subsubsection{Synthesis of PET-Based Monomers with Secondary Amines}

\section{1,4-phenylenebis(piperidin-1-ylmethanone) (PETPPI)}

PET flakes (1.02 g, $5.3 \mathrm{mmol}$ ), piperidine (PPI, $3.88 \mathrm{~g}, 45.6 \mathrm{mmol})$, and 1,5,7triazabicyclo[4.4.0]dec-5-ene (TBD, $0.06 \mathrm{~g}, 0.4 \mathrm{mmol})$ were placed in the Schlenk tube and then heated under vacuum at $95^{\circ} \mathrm{C}$ overnight $(17 \mathrm{~h})$. The homogeneous solution was then dumped in $250 \mathrm{~mL}$ of ethyl acetate and filtered. The resulting white powder was then dried in a vacuum oven at $50^{\circ} \mathrm{C}$, yielding the desired product (PETPPI) with high purity $(0.77 \mathrm{~g}, 49 \%)$

\section{1,4-phenylenebis(piperazin-1-ylmethanone) (PETPPA)}

PET flakes (1.07 g, $5.5 \mathrm{mmol})$, piperazine (PPA, $4.49 \mathrm{~g}, 52.2 \mathrm{mmol})$, and 1,5,7triazabicyclo[4.4.0]dec-5-ene (TBD, $0.07 \mathrm{~g}, 0.5 \mathrm{mmol})$ were placed in the Schlenk tube and then heated under vacuum at $135^{\circ} \mathrm{C}$ for overnight, initially. However, the piperazine sublimed out of the solution during the reaction and formed a crystal layer at the top of the Schlenk tube. This was problematic, since the sublimation left only small amounts of the reagent to be reacted. The resulting product ended up as oligomers of various sizes. Therefore, another reaction was initiated at a temperature at $135^{\circ} \mathrm{C}$ for $3 \mathrm{~h}$ to ensure that 
excess reagents were still present in the reaction to yield monomeric forms of the product. The crude solution was dumped in $250 \mathrm{~mL}$ of ether, redissolved in $5 \mathrm{~mL}$ of methanol, precipitated out again in ether, and finally filtered out. Despite the additional precautions implemented into the reaction, the white solid $(1.78 \mathrm{~g})$ contained oligomeric units, along with significant amounts of the reagent.

4.1.2.4 Synthesis of PET-Based Monomers with Non-symmetric reagents $N^{1}, N^{4}$-bis(2-hydroxyethyl)terephthalamide (PETEthA)

PET flakes (2.02 g, $10.5 \mathrm{mmol})$, ethanolamine (EthA, $11.82 \mathrm{~g}, 0.19 \mathrm{~mol})$, and 1,5,7-triazabicyclo[4.4.0]dec-5-ene (TBD, $0.1 \mathrm{~g}, 0.7 \mathrm{mmol}$ ) were placed in the Schlenk tube and then heated under vacuum at $80^{\circ} \mathrm{C}$ overnight $(15 \mathrm{~h})$. The homogeneous solution was then dumped in $250 \mathrm{~mL}$ of dichloromethane and filtered. The resulting yellow powder was then dried in a vacuum oven at $50^{\circ} \mathrm{C}$, yielding the desired product (PETEthA) with high purity $(2.41 \mathrm{~g}, 91 \%)$.

$N^{1}, N^{4}$-bis(2-(piperazin-1-yl)ethyl)terephthalamide (PETAEP)

PET flakes (1.01 g, $5.3 \mathrm{mmol}), 1$-(2-aminoethyl) piperazine (AEP, $5.95 \mathrm{~g}, 46$ $\mathrm{mmol}$ ), and 1,5,7-triazabicyclo[4.4.0]dec-5-ene (TBD, $0.07 \mathrm{~g}, 0.5 \mathrm{mmol}$ ) were placed in the Schlenk tube and then heated under vacuum at $80^{\circ} \mathrm{C}$ overnight $(18 \mathrm{~h})$. The homogeneous solution was then dumped in $250 \mathrm{~mL}$ of ethyl acetate and filtered. The resulting white sticky substance was then dried in a vacuum oven at $50^{\circ} \mathrm{C}$, yielding the desired product (PETAEP) with high purity $(0.45 \mathrm{~g}, 22 \%)$. 
$N^{1}, N^{4}$-bis(2-((2-aminoethyl)amino)ethyl)terephthalamide (PETDETA)

PET flakes (3.01 g, $15.7 \mathrm{mmol})$, diethylenetriamine (DETA, $13.87 \mathrm{~g}, 0.13 \mathrm{~mol}$ ), and 1,5,7-triazabicyclo[4.4.0]dec-5-ene (TBD, $0.12 \mathrm{~g}, 0.9 \mathrm{mmol})$ were placed in the Schlenk tube and then heated under vacuum at $80^{\circ} \mathrm{C}$ overnight $(18 \mathrm{~h})$. At the completion of the reaction, the crude solution was dumped in $250 \mathrm{~mL}$ of ethyl acetate to form a yellow oil-like substance. However, the product was crystallized by dissolving the product in small amounts $(>10 \mathrm{~mL})$ of methanol and slowly precipitating it out in $250 \mathrm{~mL}$ of ethyl acetate. Once filtered, the white powder was then dried in a vacuum oven at $50^{\circ} \mathrm{C}$, yielding the desired product (PETDETA) with high purity $(0.7 \mathrm{~g}, 13 \%)$.

\subsubsection{Synthesis of Click Functionalized PET-based Monomers} $N^{1}, N^{4}$-diallylterephthalamide (PETAllyl)

PET flakes (1.05 g, $5.5 \mathrm{mmol}$ ), allylamine (Allyl, $2.88 \mathrm{~g}, 50 \mathrm{mmol}$ ), and 1,5,7triazabicyclo[4.4.0]dec-5-ene (TBD, $0.07 \mathrm{~g}, 0.5 \mathrm{mmol})$ were placed in the Schlenk tube and then heated under vacuum at $47^{\circ} \mathrm{C}$ overnight $(20 \mathrm{~h})$. The homogeneous solution was then dumped in $250 \mathrm{~mL}$ of ethyl acetate and filtered. The resulting white powder was then dried in a vacuum oven at $50^{\circ} \mathrm{C}$, yielding the desired product (PETAllyl) with high purity $(0.92 \mathrm{~g}, 69 \%)$.

$N^{1}, N^{4}$-diallylterephthalamide (PETPro)

PET flakes (1.02 g, $5.3 \mathrm{mmol}$ ), propargylamine (Pro, $4.55 \mathrm{~g}, 83 \mathrm{mmol}$ ), and 1,5,7-triazabicyclo[4.4.0]dec-5-ene (TBD, $0.05 \mathrm{~g}, 0.4 \mathrm{mmol}$ ) were placed in the Schlenk tube and then heated under vacuum at $67^{\circ} \mathrm{C}$ overnight $(18 \mathrm{~h})$. The homogeneous solution 
was then dumped in $250 \mathrm{~mL}$ of ethyl acetate and filtered. The resulting orange sticky substance was then dried in a vacuum oven at $50^{\circ} \mathrm{C}$, yielding the desired product (PETPro) with high purity $(0.29 \mathrm{~g}, 23 \%)$.

\subsubsection{Synthesis of PET Monomers with Tertiary Functionalized Amines} $N^{l}, N^{4}$-bis(3-((3-aminopropyl)(methyl)amino)propyl)terephthalamide (PETDAMDPA)

PET flakes (3.02 g, $16 \mathrm{mmol}$ ), Diamino-N-methyldipropylamine (DAMDPA, $10.78 \mathrm{~g}, 82 \mathrm{mmol}$ ), and 1,5,7-triazabicyclo[4.4.0]dec-5-ene (TBD, $0.11 \mathrm{~g}, 0.8 \mathrm{mmol}$ ) were placed in the Schlenk tube and then heated under vacuum at $110^{\circ} \mathrm{C}$ overnight $(17 \mathrm{~h})$. The crude solution was then dumped in $250 \mathrm{~mL}$ of ether resulting in a yellow oily substance that consisted of large amounts of impurities based on H-NMR analysis. The impure product was then dissolved in $5 \mathrm{~mL}$ of methanol and precipitated out again in ether. Repeating the methanol/precipitation step two more times resulted in a yellow waxy compound. The resulting product was then dried in a vacuum oven at $50^{\circ} \mathrm{C}$, yielding the desired product (PETDAMDPA) with significant amounts of methanol and water $(1.2 \mathrm{~g}, 18 \%)$.

$N^{1}, N^{4}$-bis(6-aminopyridin-2-yl)terephthalamide (PETDAP)

PET flakes (3.84 g, $20 \mathrm{mmol}$ ), 2,6-diaminopyridine (DAP, $9.31 \mathrm{~g}, 85 \mathrm{mmol}$ ), and 1,5,7-triazabicyclo[4.4.0]dec-5-ene (TBD, $0.3 \mathrm{~g}, 2.1 \mathrm{mmol}$ ) were placed in the Schlenk tube and then heated under vacuum at $150^{\circ} \mathrm{C}$ overnight $(17 \mathrm{~h})$. The crude solution was then dumped in $250 \mathrm{~mL}$ of ethyl acetate, resulting in a dark brown solid that contained large amounts of the reagent. The impure product was then dissolved in $10 \mathrm{~mL}$ of 
dimethylformamide and precipitated out again in ethyl acetate. The resulting product was then dried in a vacuum oven at $50^{\circ} \mathrm{C}$, yielding the desired product (PETDAP) with significant amounts of methanol and water $(2.1 \mathrm{~g}, 31 \%)$.

$N^{l}, N^{4}$ - bis(2-(bis(2-aminoethyl)amino)ethyl)terephthalamide (PETTAEA)

PET flakes (1.02 g, $5 \mathrm{mmol}$ ), trisaminoethylamine (TAEA, $5.36 \mathrm{~g}, 37 \mathrm{mmol}$ ), and 1,5,7-triazabicyclo[4.4.0]dec-5-ene (TBD, $0.9 \mathrm{~g}, 0.6 \mathrm{mmol}$ ) were placed in the Schlenk tube and then heated under vacuum at $110^{\circ} \mathrm{C}$ overnight $(18 \mathrm{~h})$. The crude solution was then dumped in $250 \mathrm{~mL}$ of ethyl acetate, resulting in an orange oily solution that consisted of large amounts of impurities. The crude product was then dissolved in water and further treated through solvent exchange using ethyl acetate. The impure product was then dissolved in $10 \mathrm{~mL}$ of dimethylformamide and precipitated out again in ethyl acetate. The resulting product still contained significant amounts of water and reagents after the solvent exchange step. As a result, the product was then treated through a Kugelrohr distillation apparatus at $300^{\circ} \mathrm{C}$ for 30 minutes. The final waxy product still contained vast amounts of the reagents and water, demonstrating the great difficulty of isolating the product. In addition, the high temperature of the distillation column caused some of the product to degrade. 


\subsubsection{PET Decomposition Incorporating Reused Reagents}

The aminolysis of PET with various reagents described in the previous section required a large amount of amines to replace the use of solvents. However, the ability to separate the reagents from the organic solvent (ethyl acetate) allowed for the reuse of the remaining reagent for further PET-aminolysis reactions. Using the same reaction process described in the previous section at a 5 gram scale, the PET degradation process was restarted using the same reagents as before.

The recycled reaction was tested through the aminolysis of PET with ethylene diamine and m-xylylenediamine in a 5 gram scale. After the end of the PET-degradation reaction, the products were isolated by adding in an excess amount of ethyl acetate and filtering out the products from the supernatant. The supernatant was then separated using a rotary evaporator (rotovap) due to the differences in boiling point between the solvent and the diamines. For m-xylylenediamine, the rotovap was set at temperatures around $60^{\circ} \mathrm{C}$ due to the high boiling point of the diamine. However, the rotovap for ethylene diamine was required to be set at $40^{\circ} \mathrm{C}$ under vacuum because of the relatively lower boiling point of ethylene diamine (compared to other diamines). After the separation was complete, the diamine solution was then reused for another PET decomposition reaction $\left(100^{\circ} \mathrm{C}, 45\right.$ hours for both cases) at a 1 gram scale without the addition of TBD or diamine solution at the same operating conditions used at the 5 gram scale. The suggested amount (5\% wt) of TBD was only added if the reaction was not progressing after 5 hours. After the reaction was complete, the product was evaluated through NMR analysis. This procedure demonstrates the ability to recycle the same reagents for 
multiple PET degradation reactions, which has strong implications in improving efficiency (and reducing costs) of the reagents for large scale PET degradation reactions.

\subsection{Epoxy Hardening Process Incorporating PET-derived Amides}

The development of aromatic amides using recycled materials provides an innovative way to produce relatively inexpensive epoxy modifiers while reducing the amount of municipal waste. As discussed by McKean et al., aromatic amide based fortifiers can improve the mechanical properties of epoxy compounds. This project investigated the ability to use PET-derived amides for epoxy hardening using a solventbased epoxy hardening procedure. The solvent-based procedure focused primarily on the comparison between dissolved PET-derived amide curing agents versus the respective precursor for thermal analysis. This section centers on the materials and procedure used for the preparation of the epoxy samples for thermal characterization.

\subsubsection{Epoxy Hardening Materials}

The materials section for the epoxy hardening process is divided into two sections: epoxy copolymers and instruments. The epoxy copolymer, bisphenol A propoxylate diglycidyl ether (BAPDGE), was the primary component used for the epoxy hardening process. Four diverse PET-derived diamines, illustrated in Figure 22, (PETEthylenediamine, PET-m-Xylylenediamine, PET-Diamino-N-methyldipropylamine, and PET-Diethylenetriamine) were selected from the library developed in Table 9 for the 
epoxy hardening process. 1,1,1,3,3,3-hexa-2-fluoropropanol (HFP) was used for the solvent process in order to dissolve the PET-derived diamines into the epoxy solution. BAPDGE and HFP were purchased from Sigma-Aldrich and provided by IBM Almaden Research Facility. The only remaining copolymers involved were produced from the PET aminolysis process.
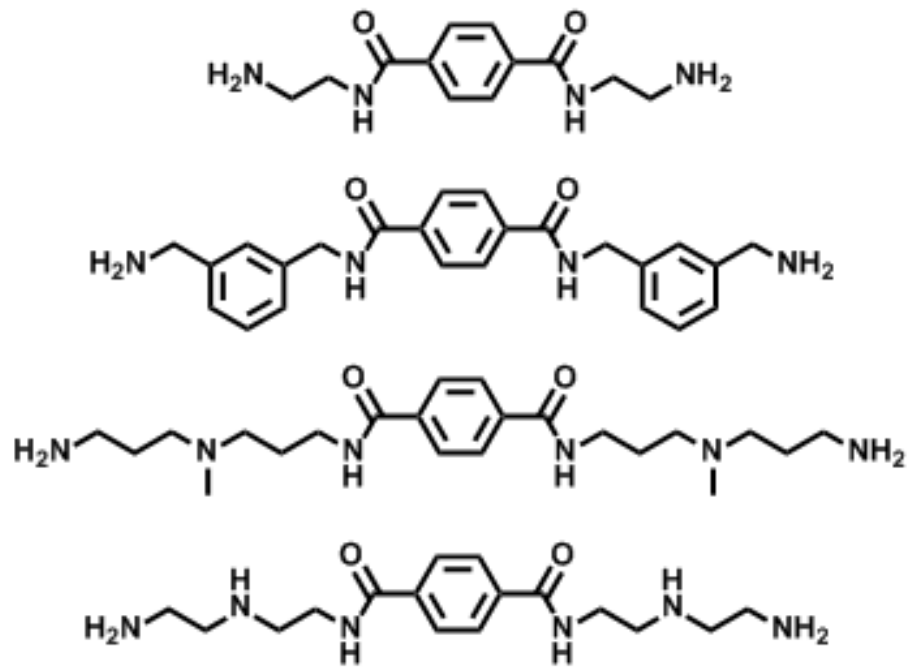

\section{PET-Ethylenediamine \\ (PETED)}

PET-m-Xylylenediamine
(PETmX)

PET-Diamino-N-methyldipropylamine (PETDAMDPA)

PET-Diethylenetriamine (PETDETA)

Figure 22. Selected PET-derived diamines used for epoxy hardening applications.

Thermal characterization was performed using a differential scanning calorimeter (DSC) and dynamic mechanical analyzer (DMA). DSC determines the glass transition temperature of the sample by calculating the changes in heat flow as a function of temperature. DMA also characterizes polymers based on the glass transition temperature by measuring the storage modulus as a function of temperature. Substantial changes in properties at higher temperatures represent physical changes between the polymer and the monomer, providing the ability to use thermal analysis to characterize epoxy hardened 
compounds. All thermal characterization tests were done at IBM Almaden Research Facility using the Q2000 DSC model and 2980 DMA model purchased from TA instruments.

\subsubsection{Epoxy Preparation for Thermal Analysis}

The method of epoxy hardening using PET-derived diamines incorporates a solvent to dissolve the PET-derived diamines into the epoxy resin for thermal analysis. Thermal analysis provides information on the curing behavior of the epoxy compounds, as temperature dependence of the properties change when the cured epoxy polymer reaches different phases based on the time-temperature transformation (TTT) profile. This section describes the preparation of the epoxy samples for DSC and DMA analysis.

The first procedure focuses on the preparation of the cured epoxy polymers for DSC analysis. Approximately 1 gram of BAPDGE was measured out and placed in a 20 $\mathrm{mL}$ vial (A). In a separate $20 \mathrm{~mL}$ vial (B), equimolar ratios of diamines (PET-derived and basic) were weighed out and added to the vial at different compositions. The goal was to determine the effects of PET-derived diamine versus its basic counterpart on the thermal properties of the epoxy polymer. Table 12 demonstrates a typical layout used for developing epoxy polymers at different ratios in a solvent system.

Approximately $2 \mathrm{~g}$ of HFP was then added to the vial B. The vial was then heated and mixed until all diamines were completely dissolved in HFP. Once cooled, the contents in vial B were poured into vial A and mixed thoroughly until the solution was uniform. The solution was then poured into an aluminum weighing dish and set on a hot 
plate at $45^{\circ} \mathrm{C}$ over two hours to evaporate out the solvent and avoid the formation of bubbles within the film without hitting the boiling point of HFP. The aluminum dish was then transferred into an oven and cured at $200^{\circ} \mathrm{C}$ over two hours under vacuum to remove any remaining solvent. The final product consisted of a brownish glasslike film, illustrated in Figure 23, which was isolated by peeling off the aluminum dish. Finally, a $0.5 \mathrm{~cm} \times 0.5 \mathrm{~cm}$ sample of the film was cut out, placed in a small aluminum plate, and run through the DSC instrument to collect thermal data.

Table 12. Experimental design for epoxy hardening of PET-derived diamines for DSC.

\begin{tabular}{|c|c|c|c|c|}
\hline $\begin{array}{c}\text { \% Composition } \\
\text { PET }\end{array}$ & BAPDGE & PET-ED & ED & HFP \\
\hline $100 \%$ & $1 \mathrm{~g}$ & $0.274 \mathrm{~g}$ & $0 \mathrm{~g}$ & $2 \mathrm{~g}$ \\
\hline $70 \%$ & $1 \mathrm{~g}$ & $0.191 \mathrm{~g}$ & $0.020 \mathrm{~g}$ & $2 \mathrm{~g}$ \\
\hline $50 \%$ & $1 \mathrm{~g}$ & $0.137 \mathrm{~g}$ & $0.033 \mathrm{~g}$ & $2 \mathrm{~g}$ \\
\hline $30 \%$ & $1 \mathrm{~g}$ & $0.082 \mathrm{~g}$ & $0.046 \mathrm{~g}$ & $2 \mathrm{~g}$ \\
\hline $0 \%$ & $1 \mathrm{~g}$ & $0 \mathrm{~g}$ & $0.066 \mathrm{~g}$ & $2 \mathrm{~g}$ \\
\hline
\end{tabular}
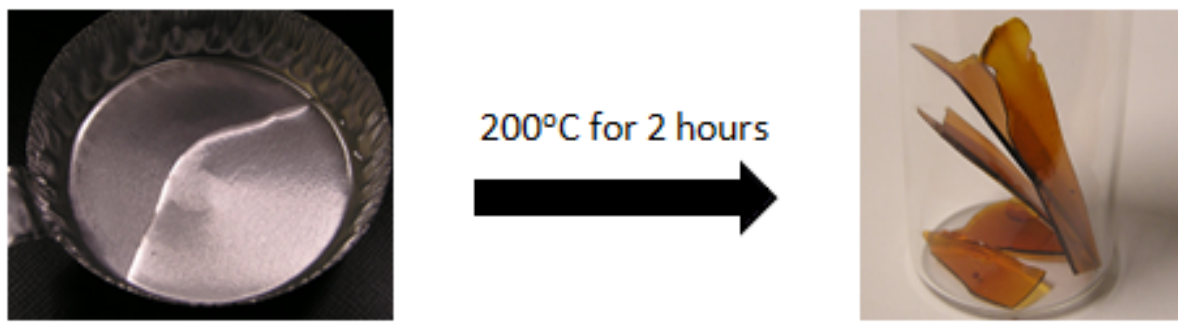

Figure 23. Illustration of epoxy hardened polymer for DSC analysis. 
The DSC analysis measures the glass transition temperature of the epoxy compound by measuring the heat flow through the polymer as a function of temperature. As the temperature rises, the epoxy polymer reaches vitrification, at which point the bonds of the polymer become loose and the heat transfer through the polymer reaches a high point. This effect was exploited using the DSC to determine the glass transition temperature at the fully cured state of the polymer by measuring the specific heat as a function of temperature. However, it was important to expose the sample to several heat runs to ensure that it reached the maximum level of cross-linking and the value of the glass transition state was that for the fully cured state (Figure 24). As a result, each sample analyzed through the DSC needed to be heated and analyzed several times to determine the closest value for the glass transition temperature at the fully cured state.

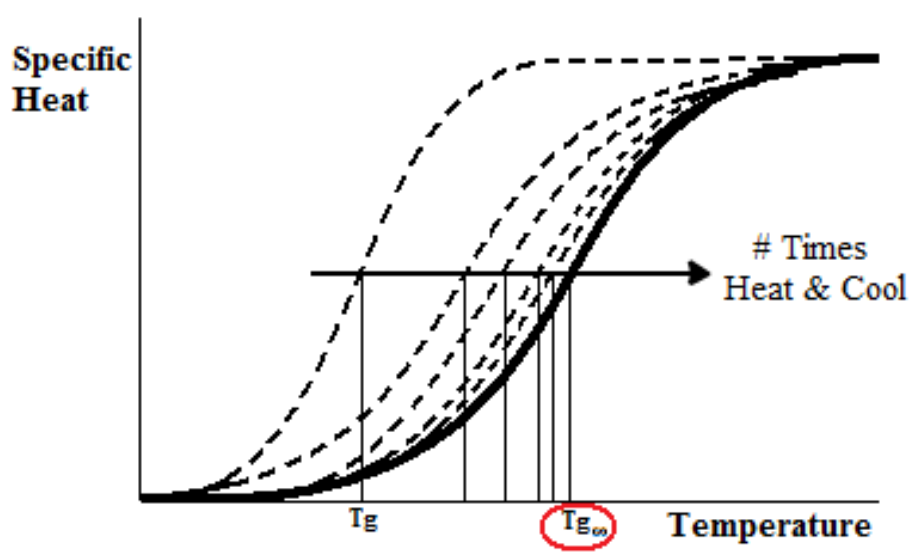

Figure 24. Glass transition temperature at fully cured state for DSC analysis. 
The second procedure focused on the preparation of the cured epoxy polymers for DMA studies. Approximately 0.5 gram of BAPDGE was measured out and placed in a $20 \mathrm{~mL}$ vial (A). In a separate $20 \mathrm{~mL}$ vial (B), equimolar ratios of diamines (PETmodified vs. basic) were weighed out and added to each associated vial at two different compositions: $0 \%$ and 100\% PET-derived diamine to basic diamine ratios, as presented in Table 13. Approximately 2 grams of HFP were added to vial B, which was then heated and mixed in order to fully dissolve the diamines into solution. The contents in both vials were then combined and mixed thoroughly to form a uniform solution. The contents of the solution were then pipetted onto a $6 \mathrm{~cm}$ by $0.5 \mathrm{~cm}$ thin glass fiber strip on top of a glass plate until the glass fiber strip could no longer absorb the solution (Figure 25 (a)). The solvent was evaporated out by placing the glass plate in the oven at $50^{\circ} \mathrm{C}$ for 2 hours under vacuum. The samples were then cured at $200^{\circ} \mathrm{C}$ in an oven for 2 hours under vacuum as well, where the solution turned into a glass-like layer (Figure 25 (b)). The glass fiber strips were then carefully removed from the glass plate after cooling and entered into the DMA for analysis.

Table 13. Experimental design for epoxy hardening of PET-derived diamines for DSC

\begin{tabular}{|c|c|c|c|c|}
\hline \% Composition PET & BAPDGE & PET-ED & ED & HFP \\
\hline $100 \%$ & $0.5 \mathrm{~g}$ & $0.137 \mathrm{~g}$ & $0 \mathrm{~g}$ & $2 \mathrm{~g}$ \\
\hline $0 \%$ & $0.5 \mathrm{~g}$ & $0 \mathrm{~g}$ & $0.033 \mathrm{~g}$ & $2 \mathrm{~g}$ \\
\hline
\end{tabular}




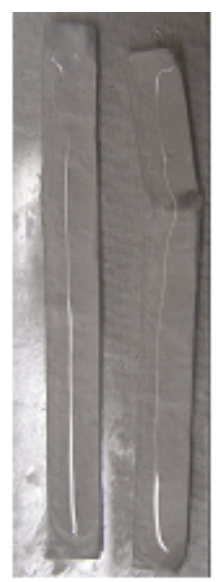

(a)

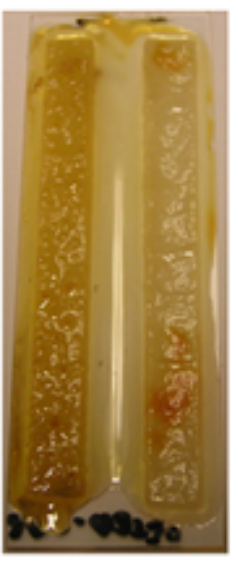

(b)

Figure 25. DMA epoxy samples (a) after sample preparation and (b) post-cured.

Characterization through DMA provides information about the glass transition temperature, as well as the cross-linking behavior as the polymer goes through devitrification to reach the elastomeric state based on the TTT diagram. The transition from the glass state to an elastomeric state can be visualized by measuring the modulus of a thermoset sample versus temperature, as illustrated in Figure 26. Any abnormal crosslinking resulting from curing difficult compounds can be accounted for by the shape of the DMA profile. This is done to ensure that the PET-derived diamines correctly behave as modifiers for epoxy hardening. The molecular weight between cross-links can also be calculated based on the value of the modulus at the elastomeric region. However, it should be pointed out that the particular instrument calculates a relative modulus and thus is used specifically to determine the thermal behavior of the composite samples. 


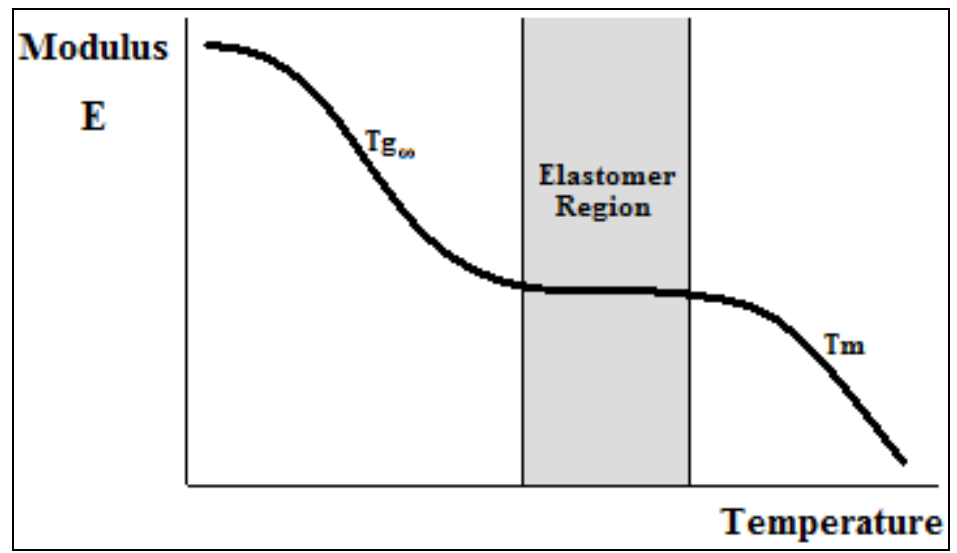

Figure 26. Expected DMA result for fully cured epoxy polymer

\subsection{Methods and Materials Summary}

This section listed the respective processes for the aminolysis of PET into new monomers using various different reagents and the incorporation of these monomers for epoxy hardening. Incorporating organocatalysts to degrade plastic waste polymers in solvent-less systems is a creative way of removing waste while following the principles of green chemistry. In addition, the ability to form a library of structurally strong aromatic amides may be important for the application of PET-derived diamines in epoxy hardening. Using thermal tests, quantitative measurements were used to test the curing behavior of the epoxy polymers with the addition of these aromatic amides. This chapter provided the backbone for how the PET degradation products were formed, analyzed, and applied. 


\section{CHAPTER FIVE \\ PET AMINOLYSIS RESULTS}

The main objective of this project was to develop an extensive library of aromatic amides from the reaction between PET waste and amines. This chapter begins by explaining how the spectrums developed from proton nuclear magnetic resonance spectroscopy (H-NMR) for these PET-based monomers were analyzed. Additionally, an in-depth analysis of the performance of the PET aminolysis reactions was conducted to determine any trends between the reaction performance and the properties of the reagents. Table 14 provides an overall synopsis of each PET aminolysis reaction tested along with the performance of each reaction based on yield. Furthermore, this chapter delves into the results concerning the ability to reuse reagents, specifically using PETethylenediamine and PET-m-xylylenediamine, for conducting multiple PET degradation reactions. Finally, the last section focuses on the importance of the process design along with these novel monomers in the global scale. 
Table 14. Summary of PET aminolysis reactions

\begin{tabular}{|c|c|c|c|c|}
\hline$\#$ & PET-Based Product & Chemical Structure & Isolated & \% Yield \\
\hline 1 & PETED & & Yes & 99 \\
\hline 2 & PETDAB & & Yes & 85 \\
\hline 3 & PETDAH & & Yes & 93 \\
\hline 4 & PETmX & & Yes & 72 \\
\hline 5 & PETpX & & Yes & 75 \\
\hline 6 & PETABA & & Yes & 86 \\
\hline 7 & PETPPD & & Yes* & $>100$ \\
\hline 8 & PETPPI & & Yes & 49 \\
\hline 9 & PETPPA & & No & $>100$ \\
\hline 10 & PETEthA & & Yes & 91 \\
\hline 11 & PETAEP & & Yes & 22 \\
\hline 12 & PETDETA & & Yes & 13 \\
\hline 13 & PETAllyl & & Yes & 69 \\
\hline 14 & PETPro & & Yes & 23 \\
\hline 15 & PETDAMDPA & & Yes & 18 \\
\hline 16 & PETDAP & & Yes & 30 \\
\hline 17 & PETTAEA & & No & 13 \\
\hline
\end{tabular}




\subsection{H-NMR Analysis for PET-Derived Monomers}

The formation of the aromatic amide plays a key role in identifying the product through H-NMR analysis. Due to the electron pulling nature of the amide group, the signal of the protons on the aromatic amide appears at a higher chemical shift in comparison to the peaks of the reagents. Using the H-NMR spectrum for the PETethylenediamine (PETED) product, illustrated in Figure 27, the peaks for the protons on the aromatic group of the amide are located at a chemical shift $(\delta)$ around $7.9 \mathrm{ppm}(\mathrm{A})$ on the spectrum. Although the spectrum only labels half of the compound, the symmetric nature of the PET-based monomers implies that both sides of the compound have equivalent peaks, thus simplifying the H-NMR analysis for each compound. The NMR spectrum also includes peaks for deuterated DMSO $(\delta=2.5 \mathrm{ppm})$ and water $(\delta \approx 3.3$ ppm). These peaks are consistent for all H-NMR analysis done on PET-based monomers.

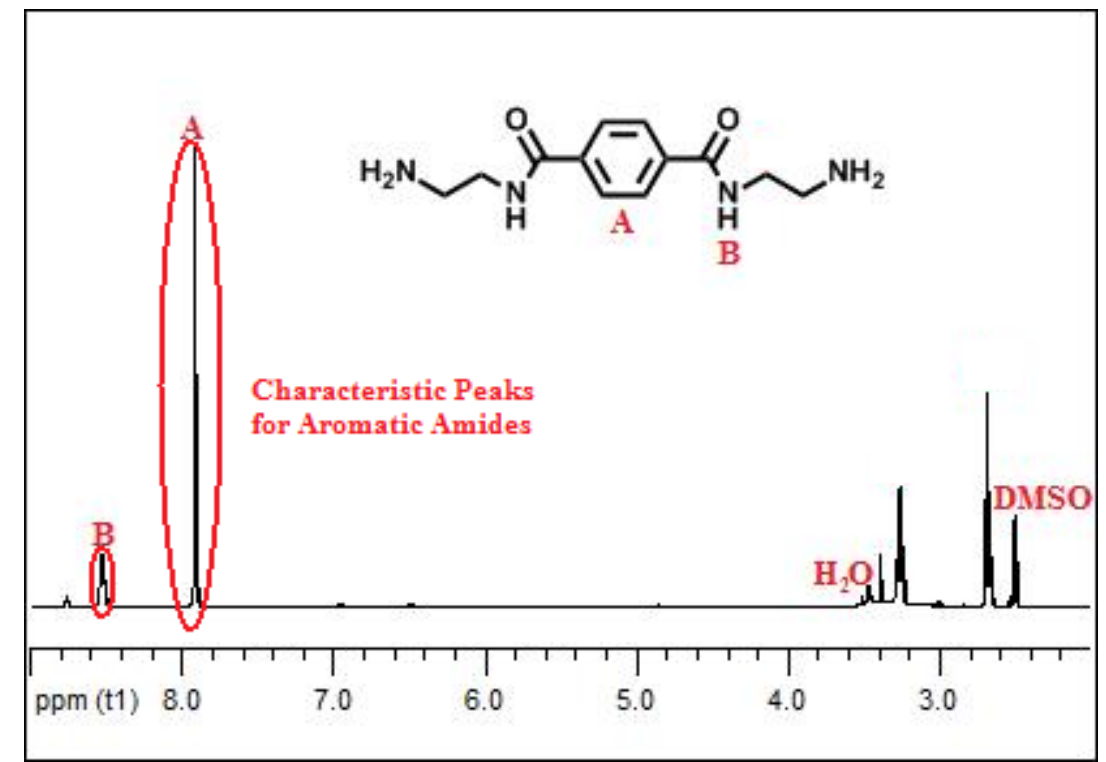

Figure 27. H-NMR spectrum of PETED used to illustrate the characteristic peaks specific to the aromatic amide group for each PET-based monomer. 
Although the H-NMR for PETED looked clean, the production of oligomeric products is a concern when developing PET-based monomers with diamine reagents. The peak at a chemical shift at $8.8 \mathrm{ppm}$, shown in Figure 27, revealed oligomers in the product. However, the amount of oligomers was nearly insignificant compared to the amount of monomers present in the sample. The presence of the oligomers does illustrate the importance of using excess reagents for these reactions.

\subsection{Performance Analysis of PET Aminolysis Reactions}

Using the procedures detailed in Chapter 4 to develop the PET-based monomers, the H-NMR data and reaction yields can be analyzed with the type of reagent to find trends between performance and the type of reagents used. Table 14 provides a summary of all the PET aminolysis reactions executed in Chapter 3. The table also provides information on the performance of the reaction (i.e., \% yield) and whether the product was successfully isolated from the reagents. This section presents the general trends and obstacles in the performance of the PET aminolysis reaction using the "green" design scheme.

\subsubsection{PET Aminolysis with Aliphatic and Aromatic-Containing Diamines}

The PET degradation reactions involving the aliphatic and aromatic amines generally performed very well with high purity, as demonstrated by the H-NMR spectra (Figures 28 and 29) and yields above $72 \%$. The high performance was related to the reactivity of the primary amine on these reagents. Aliphatic primary amines are more 
effective nucleophiles than phenyl and secondary amines due to the lack of steric hindrances from the protons on the primary amine. In addition, there are no other functional groups on these amines that would inhibit aminolysis from occurring in the reaction. Therefore, reactions between PET and the aliphatic amines should have extremely high levels of conversion (>85\%). Similarly, the diamine reagents containing phenyl groups performed well in the aminolysis with PET with yields generally over $72 \%$. In addition, the aminolysis reaction with aminobenzylamine (ABA) demonstrated the ability to target the more reactive benzyl amine over the phenyl amine. This truly demonstrates the ability of TBD to target certain functional groups on the basis of reactivity under particular reaction conditions. By increasing the reaction temperature to $150^{\circ} \mathrm{C}$, the phenyl amine groups could be targeted, as demonstrated by the success in producing PETPPD. However, through the use of multiple washing steps, PETPPD still contained some reagent impurities in the product. This may be due to the affinity for the reagent to remain bound to the product as a result of the pi interactions between the phenyl groups. Despite the minor complexity in the isolation of PETPPD, the synthesis of PET-derived monomers using aliphatic and aromatic diamines generally produced well-defined crystalline products with high yields. 
(a)

(b)

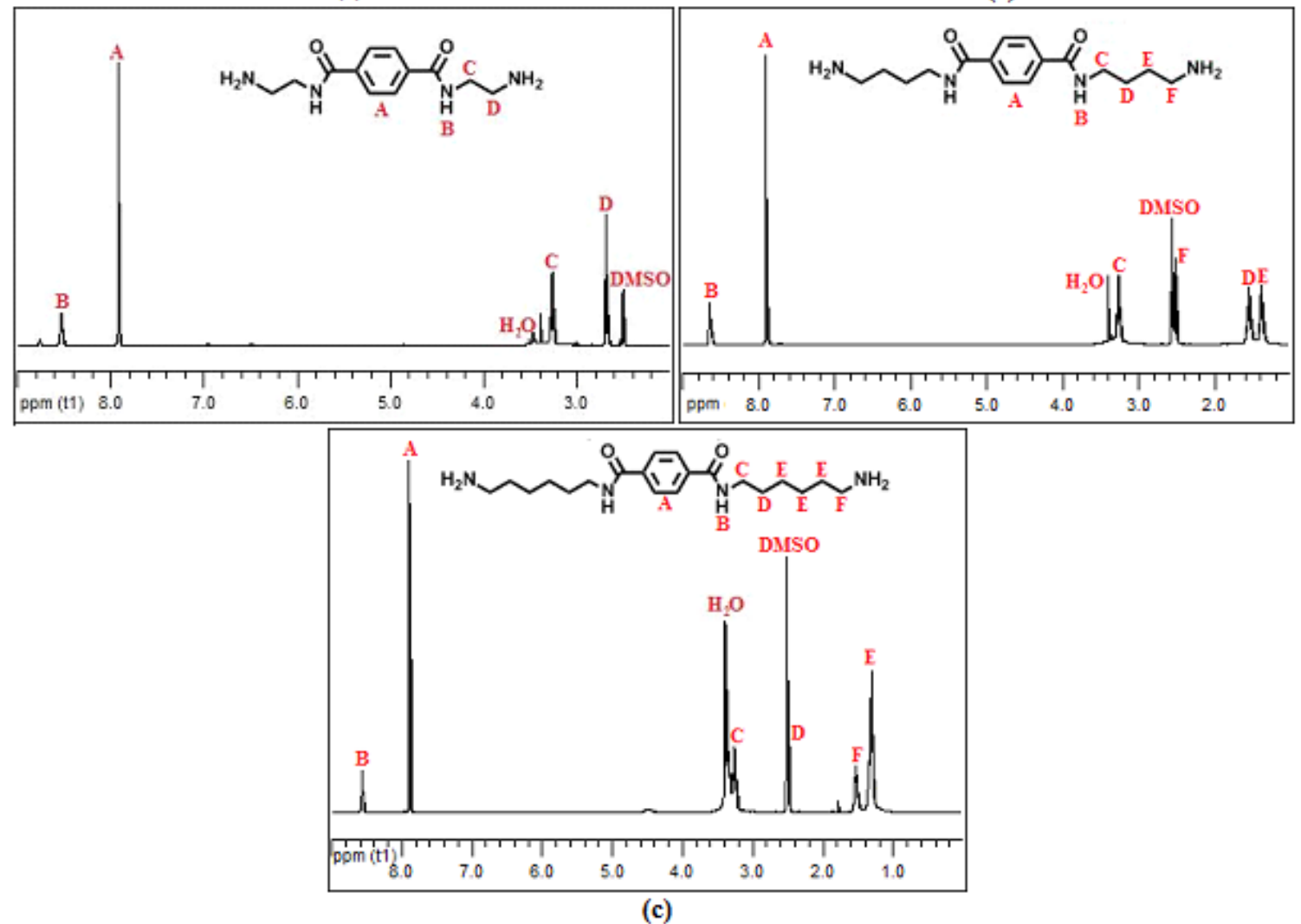

Figure 28. H-NMR characterization of the aliphatic PET-based diamines; (a) PETED, (b) PETDAB, and (c) PETDAH. 
(a)

(b)

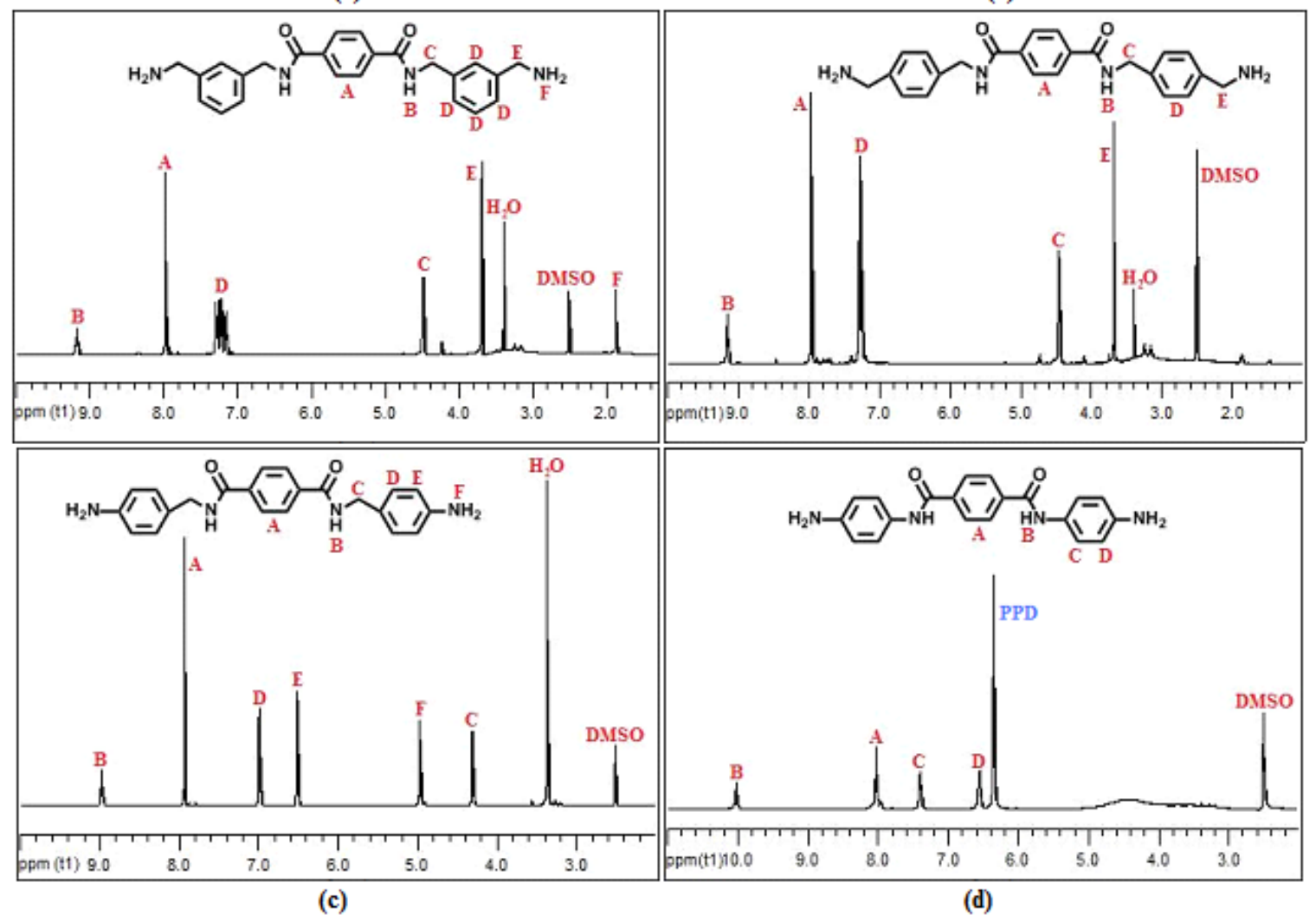

Figure 29. H-NMR characterization of the phenyl containing PET-based diamines; (a) PETmX, (b) PETpX, (c) PETABA, and (d) PETPPD.

\subsubsection{PET Aminolysis with Non-Symmetric Amines}

The formation of PET-ABA by effectively targeting the functional group based on reactivity opened the door for the development of specific monomers from nonsymmetric reagents through adjusting the reaction conditions. The successful production of the first compound, PETEthA, demonstrated the ability to specifically target the more nucleophilic amine site over the alcohol in the formation of these monomers at high yields (91\%), as illustrated by the H-NMR spectrum located in Figure 30. In addition, 
the H-NMR spectra in Figure 30 also demonstrated the ability to form PET-based monomers by targeting the more reactive primary amines over secondary amines in the successful production of PETAEP and PETDETA. Interestingly, the development of PETDETA using organocatalysis demonstrated the ability to develop one type of monomer rather than a mixture, as illustrated in the aminolysis of PET by Spychaj et al. However, it is important to note that the amorphous nature of PETAEP and the hygroscopic nature of PETDETA made it significantly more difficult to purify using the desired trituration process, resulting in the low yields of the product.

(a)

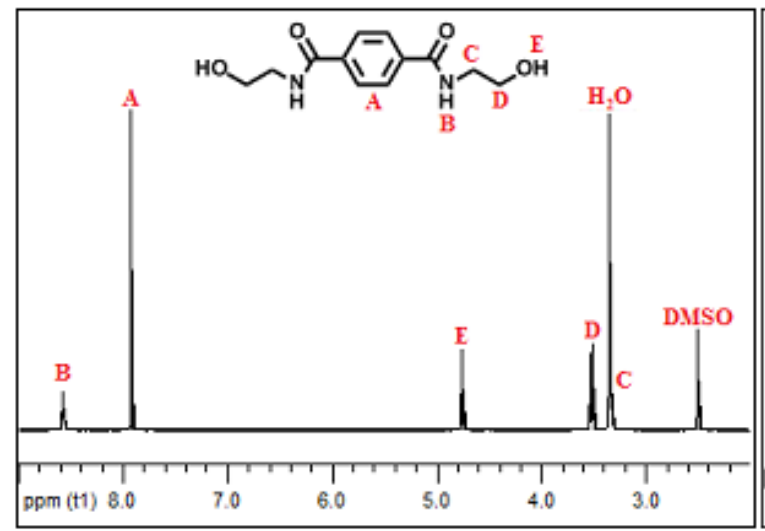

(b)
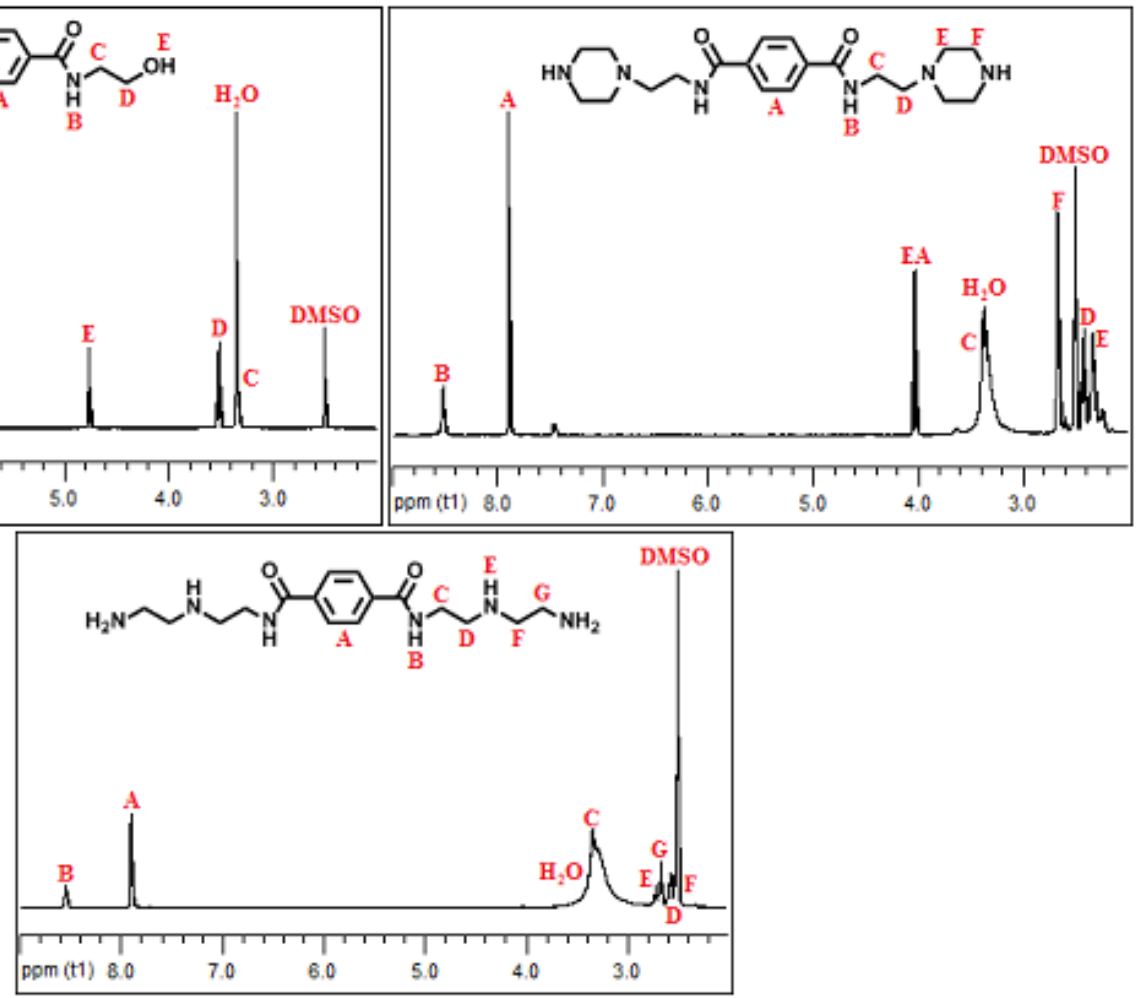

(c)

Figure 30. H-NMR characterization of non-symmetric PET-based monomers; (a) PETEthA, (b) PETAEP, and (c) PETDETA. 


\subsubsection{PET-Aminolysis with Click-Functionalized and Secondary Amines}

The organocatalytic aminolysis of PET with click-functionalized amines and secondary amines was successful as well. Both click-functionalized amines (PETAllyl and PETPro) were produced and isolated with high purity using low temperatures (Figure 31) due to the limitations imposed by the low boiling points of the reagents. However, as demonstrated by the yields for both PETAllyl and PETPro, monomers with amorphous properties (PETPro) versus crystalline properties (PETAllyl) experienced considerable losses through the trituration process, as the product would not easily precipitate out in solution. The PET reactions with secondary amines yielded mixed results, as PETPPI formed a nice crystalline product with moderate yields (49\%) (Figure 32), while PETPPA could not be successfully produced using the intended experimental design. During the reaction, piperazine sublimed out of solution, leaving minute amounts of reagent in the reaction volume. In addition, the product had solubility properties similar to the reagent, making it very difficult to isolate. However, the presence of the peaks at a chemical shift of $7.4 \mathrm{ppm}$ did show promise that the compound can be developed using a modified procedure to successfully produce PETPPA. 


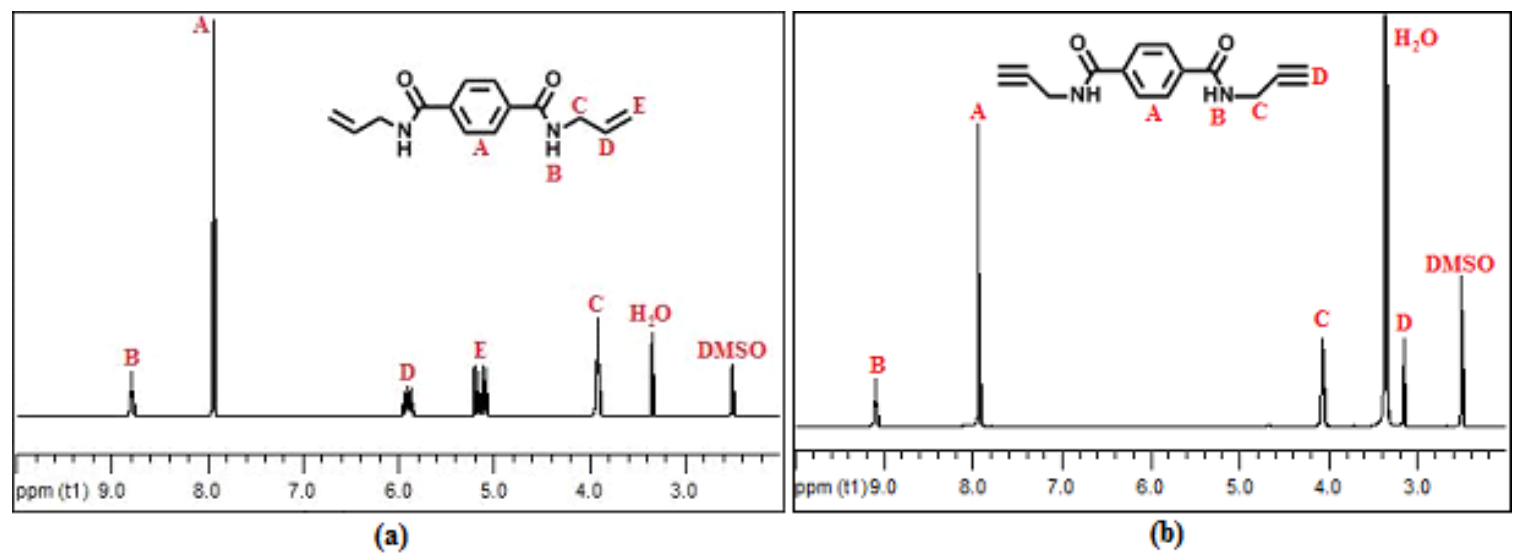

Figure 31. H-NMR characterization of PET-based monomers with clickfunctionalization; (a) PETAllyl and (b) PETPro.

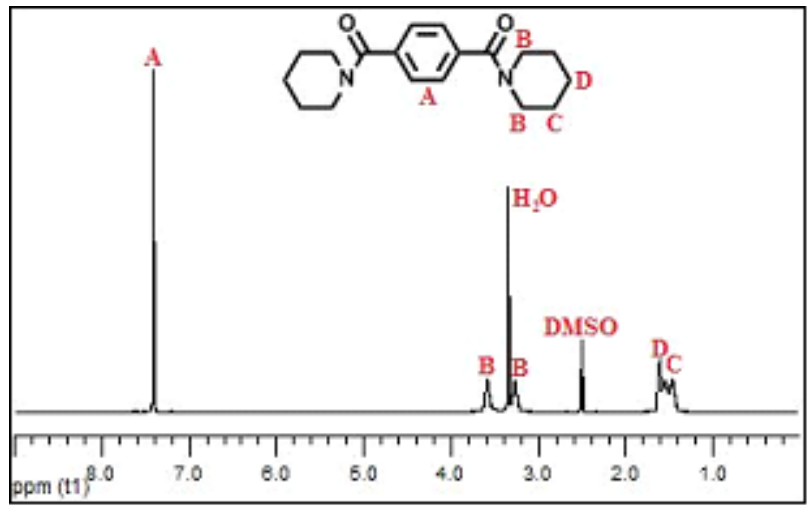

(a)

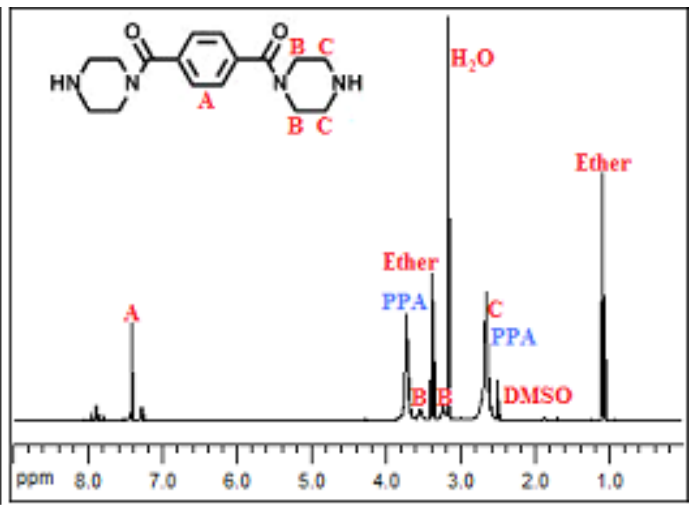

(b)

Figure 32. H-NMR characterization of PET-based monomers using secondary amines; (a) PETPPI and (b) PETPPA. 


\subsubsection{PET Aminolysis with Tertiary-Functionalized Amines}

Finally, the formation of PET-derived monomers with tertiary-functionalized amines is interesting because of the water solubility characteristics of tertiary amines as well as the ability to form charged quaternary amines that can be used for a variety of different applications. The formation of the oily layer when dumped in the organic solvent, due to the hygroscopic nature of these particular products, makes it very difficult to isolate using a basic trituration procedure. Additional precipitation steps are required to completely isolate the product from the reagent, which leads to the low yields in the product.

Both PETDAMDPA and PETDAP were successfully produced and isolated, as demonstrated by the H-NMR spectrum located in Figure 33. For PETDAP, because the tertiary amine is lodged in the aromatic ring, any water solubility effects provided by the basic tertiary amine were negated due to the structural rigidity of the compound. An interesting characteristic of PETDAMDPA, however, lies in the fact that the dimer form, rather than the monomeric form, is isolated (Figure 34) when the trituration and precipitation steps require ethyl acetate instead of ether. Since PETDAMDPA contains both water soluble parts (tertiary amine) and insoluble parts (aromatic amide), having a dimer (Figure 34) would enable the compound to have a larger ratio of insoluble segments to water-soluble segments $(2: 3)$ than the monomeric form $(1: 2)$. Therefore, the dimer is less hygroscopic and much easier to crystallize out than the monomeric form. This also explains why the dimer form is insoluble in water, making it the less desirable product for potential applications, such as interfacial polymerization. Despite the success 
of the previous two compounds, PETTAEA could not be produced and isolated as water/methanol, and the reagents were difficult to remove using several different purification techniques (solvent exchange, precipitation, etc.). Even when the crude product was treated using a Kugelrohr distillation apparatus at a high temperature, small traces of impurities were still found in the product, along with the presence of some degraded products caused by the high temperatures required for distillation. Despite the extra attention required to obtain these tertiary-amine functionalized compounds, these monomers do provide special properties that make it more valuable than the other PETbased monomers. 
(a)
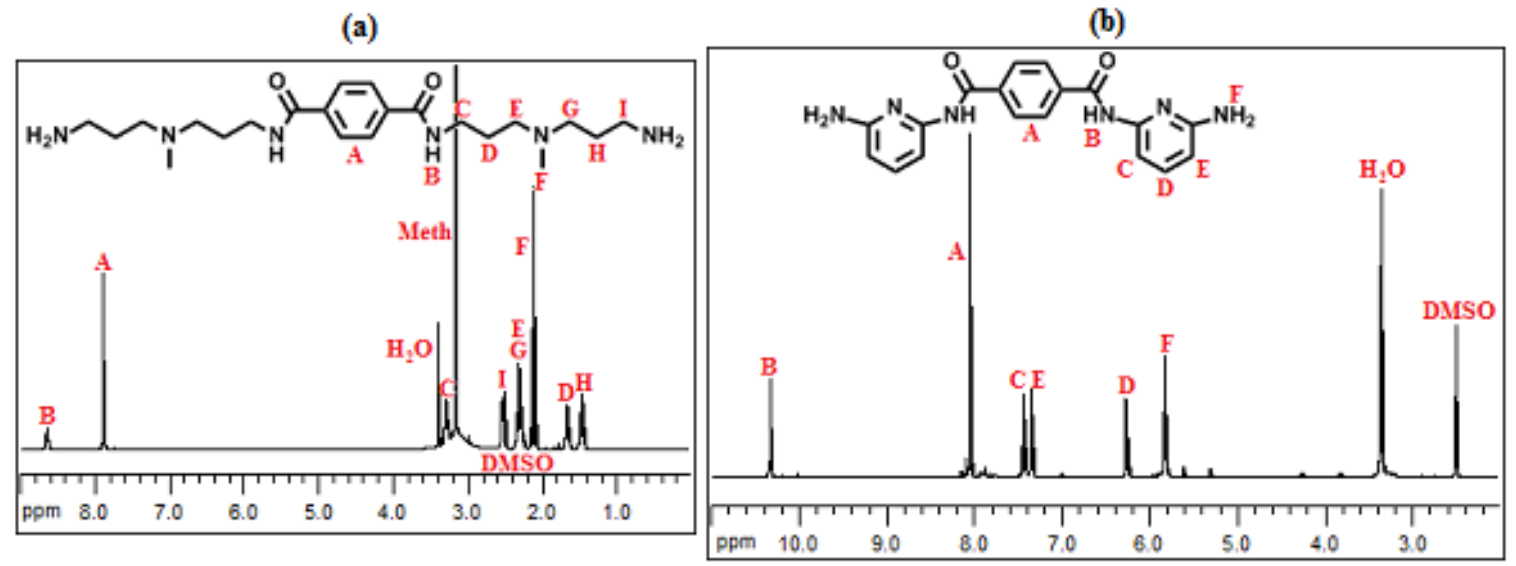

(c)

Figure 33. H-NMR characterization of PET-based monomers with tertiary-functionalized amines; (a) PETDAMDPA, (b) PETDAP, and (c) PETTAEA. 


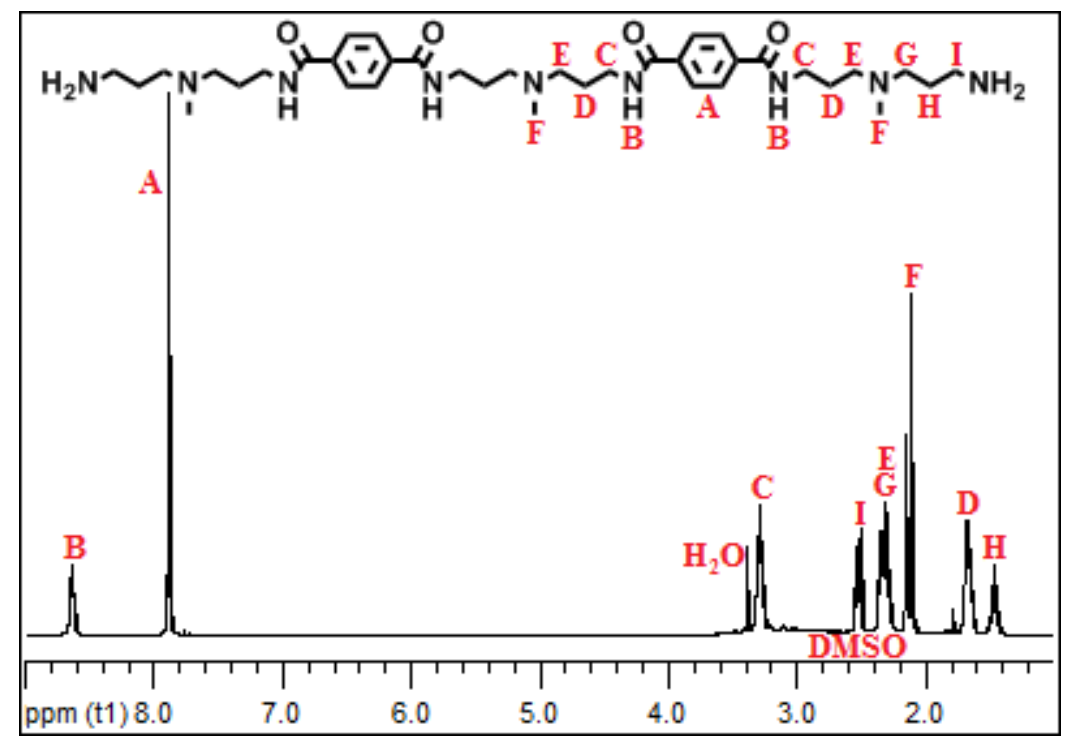

Figure 34. H-NMR spectrum for PETDAMDPA (dimer).

\subsection{Recycling of Amine Reagents for PET-Aminolysis}

The decomposition of PET using organocatalysis has facilitated the creation of new monomers by simply adding excess amounts of various amines. However, the cost of using excess amounts of amines negates the advantages of producing monomers from waste products. Recycling the reagents after forming PET products may make this process considerably more cost effective. Using the procedure detailed in Chapter 4, the recycling of both m-xylylenediamine $(\mathrm{mX})$ and ethylenediamine (ED) were investigated to perform additional PET degradation reactions.

The first reaction tested to determine the ability to recycle amine reagents for further PET degradation consisted of $\mathrm{mX}$. Using the reaction scheme illustrated in Figure 
35 , mX was separated from the ethyl acetate solution after the purification of initial PETmX ( $\sim 5 \mathrm{~g}$ scale) through the use of the rotovap. $\mathrm{mX}$ was tested first because $\mathrm{mX}$ and ethyl acetate can be easily separated due to their considerable differences in volatility. The next step was performing the identical reaction at a 1 gram scale of PET using only the recycled $\mathrm{mX}$ solution (which still contained TBD from the previous reaction). The reaction produced a white product at $64 \%$ yield with viscid properties. The results, illustrated from the H-NMR spectrum displayed in Figure 36, demonstrated the successful production of PETmX using recycled reagents. However, the viscid properties were likely due to the small impurities seen in the NMR spectrum at a chemical shift around 4.2 and $1.9 \mathrm{ppm}$. The impurities within the product may have been degraded TBD byproducts formed due to excessive use of TBD. Despite the impurities, this process did show high potential for recycling excess reagents to further produce PETdegraded products.

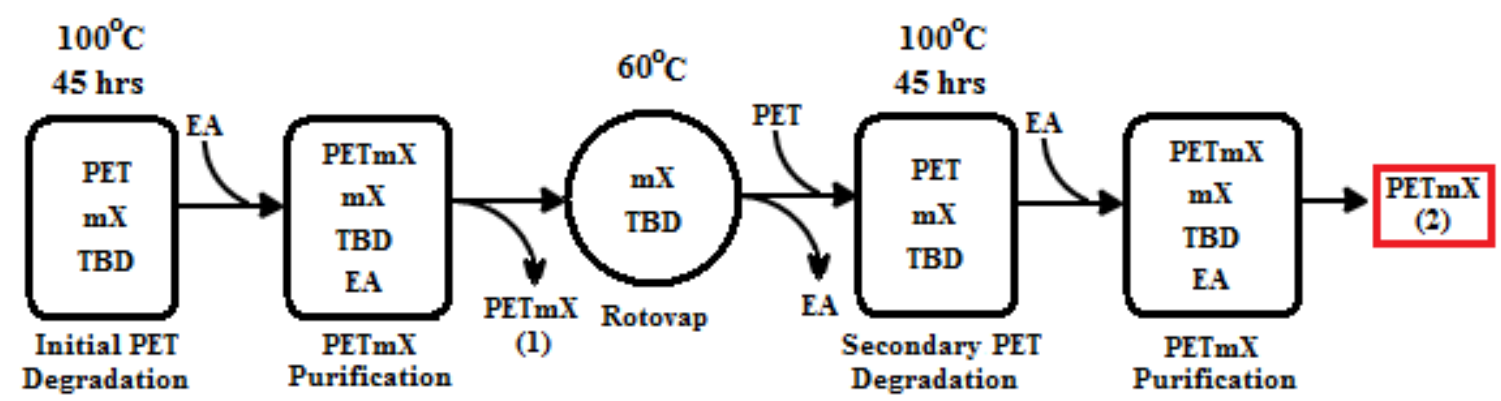

Figure 35. Reaction scheme for using recycled $\mathrm{mX}$ to perform a PET aminolysis reaction. 


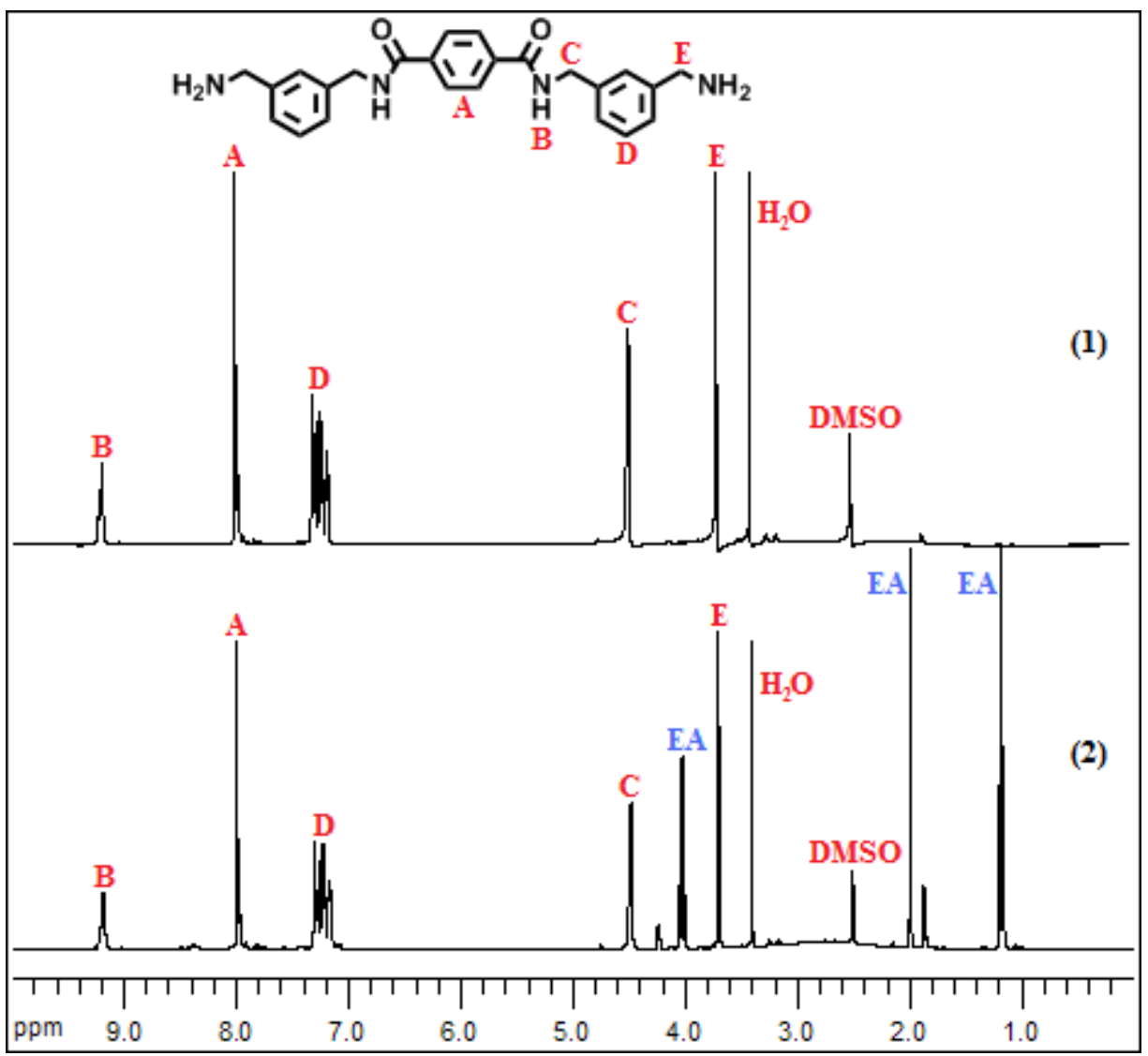

Figure 36. NMR analysis of PETmX product using (1) pristine $\mathrm{mX}$ and (2) recycled $\mathrm{mX}$

The recycling of ethylenediamine for additional PET degradation reactions was also investigated to further demonstrate the potential efficient use of reagents. The reaction scheme for producing PETED using recycled ethylenediamine (Figure 37) differed considerably from the m-xylylenediamine scheme. First, the rotovap temperature was set to $40^{\circ} \mathrm{C}$ instead of the $60^{\circ} \mathrm{C}$ used in the m-xylylenediamine process due to the lower volatility of ethylenediamine compared to m-xylylenediamine. In addition, the secondary PET degradation reaction did not turn homogeneous after 10 hours. Thus, additional TBD was added (5 wt. \% of PET) in order to allow the reaction 
to proceed. After 45 hours, the reaction created a white powder with a yield larger than 100\%. The H-NMR spectrum of both PETED products in Figure 38 demonstrated the formation of PETED using recycled ethylenediamine. However, for the PETED sample produced from recycled ethylenediamine, PETED oligomers were present along with the monomers. The reason for the generation of PETED oligomers was the limited amount of ED remaining after the rotovap step. This could be remedied by adding more ED to the PET degradation reaction to ensure that it occurred at high excess of ED. In addition, the high amounts of impurities in the secondary PETED sample explained why the yield was higher than expected. The inability of the secondary PET degradation to occur without the addition of TBD may have been caused by excessive degradation of TBD from the initial PETED process. Consequently, the degraded TBD byproducts may have accounted for the large quantities of impurities in the PETED sample. In conclusion, both recycled m-xylylenediamine and ethylenediamine successfully demonstrated the ability to reuse reagents for PET aminolysis. However, processes incorporating recycled reagent for PET aminolysis reactions need to consider ways of separating the PET-based products from degraded TBD byproducts.

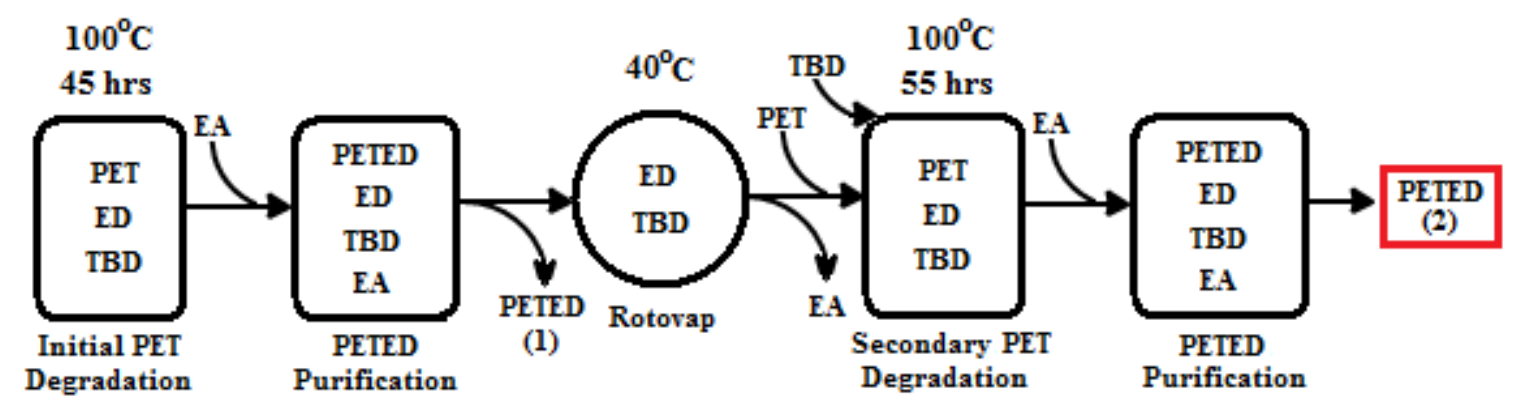

Figure 37. Reaction scheme for using recycled ED to perform a PET aminolysis reaction. 


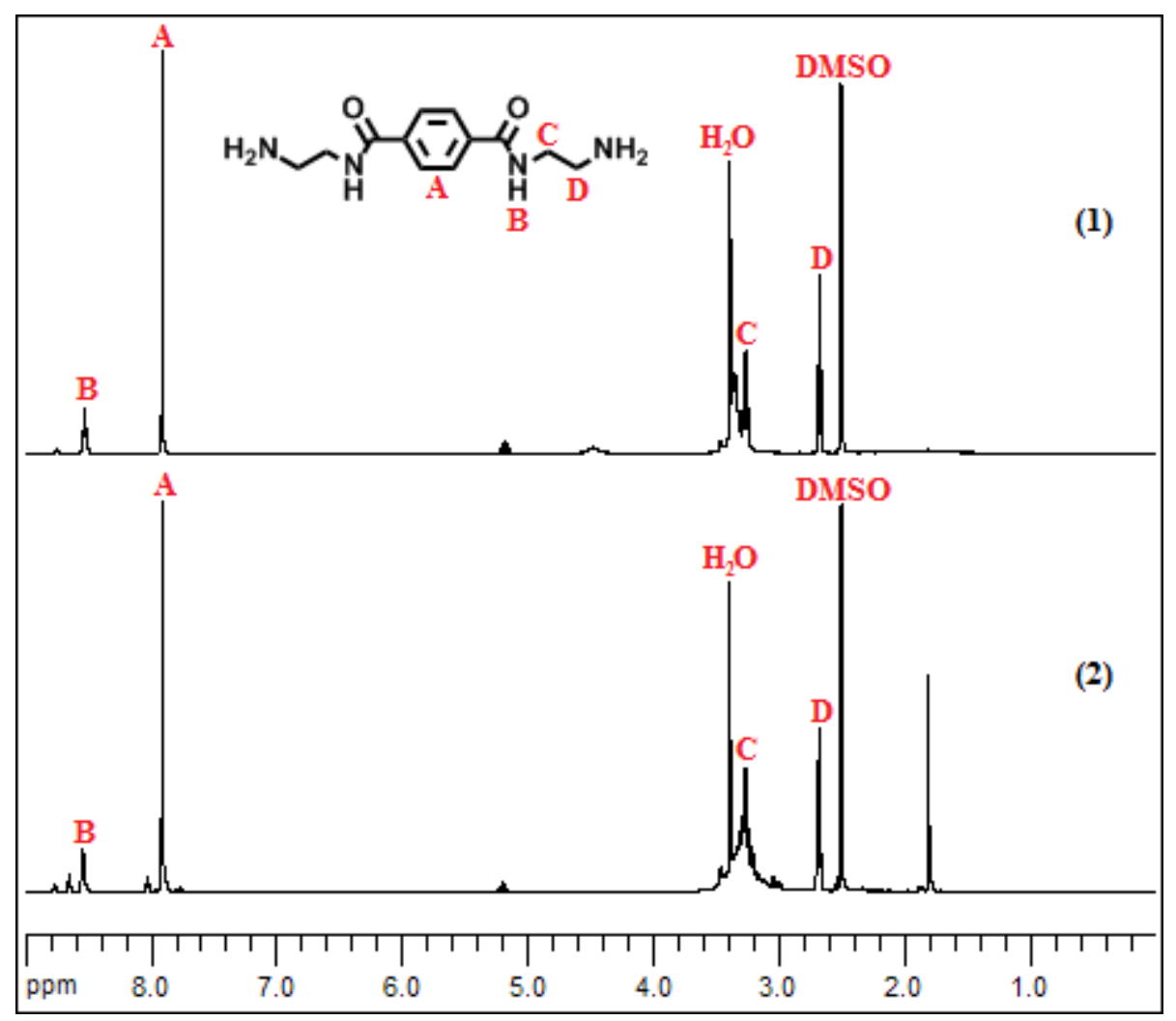

Figure 38. NMR analysis of PETED product using (1) pristine ED and (2) recycled ED 


\subsection{Discussion}

This project demonstrated the ability, through organocatalysis, to develop a library of specialty monomers with various functional groups using PET waste products. Consequently, the process as well as the monomers should be discussed to signify their importance on the global scale. This section goes over the significance of this process toward the environment, the additional studies that should be done to optimize process conditions, and, finally, the characteristics of the PET-based monomers that make it useful for potential applications.

\subsubsection{Environmental Significance of Developing PET-Based Monomers}

The overwhelming quantities of PET products released into the environment have been detrimental to the ecosystem, as these products cannot be readily eradicated. Developing specialty monomers from PET waste offers an alternative means of treating the waste with the potential to incorporate these monomers for high-value applications. The ability to develop these monomers for high-value applications in turn may provide an added economic incentive to collect and reduce the amount of PET waste in the environment. In addition, the goal is to also apply these monomers in uses with long life spans, such as coatings, to avoid these PET products from entering the landfill.

As the world becomes more environmentally conscious, there is a drive to develop more environmentally friendly processes for large-scale manufacturing of chemicals. The design scheme for the PET aminolysis using organocatalysis provides a way of developing new monomers at low operating conditions and solvent-less reaction 
conditions with the ability to recycle reagents and trituration solvents for additional PET reactions. Although these properties may seem environmentally insignificant at the lab scale, using this practical design at a manufacturing scale provides significant advantages resulting from its simplicity and ability to comply with growing environmental restrictions.

Of all the monomers developed, however, certain compounds carry more weight, especially on the environment. Looking at the results summarized in Table 14, there is clearly a divide in productivity based on the properties of the compounds. Amorphous and hygroscopic products generally produce low yields and require additional purification steps to isolate. Environmentally, the development of these monomers is less favorable, as considerably more solvent and energy need to be expended to reach the level of production seen with the crystalline monomers. The ability of the crystalline products to crash out of solution quickly and effortlessly truly demonstrates the power of this process, especially for large scale production.

\subsubsection{Process Optimization}

The main objective of this project was to specifically demonstrate the ability to develop a library of compounds by reacting PET waste with varying reagents. However, due to the time limitations of this study, process optimization was not fully investigated. Kinetic studies of these aminolysis reactions should be performed to highlight the roles that certain conditions play on the ability to perform these reactions. The three main factors that should be seriously considered include the reaction temperature, TBD 
concentration, and reagent concentrations. The ability to perform the PET aminolysis reactions with allylamine and propargylamine at temperatures well below $100^{\circ} \mathrm{C}$ is rather intriguing, as it might provide a way of reducing the energy requirements, leading to a greener process for the reaction with other reagents. Kinetic studies with TBD concentration should also be investigated to find the minimal amount of TBD necessary to perform the PET aminolysis reaction. Finally, the effects of reagent concentration on these PET aminolysis reactions should be determined to provide a way of controlling oligomeric formation. The process optimization is imperative, especially at manufacturing scales, to reduce costs while making the process considerably greener.

\subsubsection{Importance of Properties PET-Based for Potential Applications}

The specialty monomers derived from PET contain extraordinary properties that make it enticing for various applications. First, the added rigidity provided by the phenyl ring on the aromatic amide enhances the structural rigidity of the monomer. Second, the amide groups provide additional structural support through hydrogen bonding. Finally, the terephthalic structure forces these monomers to become bifunctional monomers. The combination of these properties makes these monomers very enticing for condensation polymerization reactions, as the rigidity and hydrogen bonding properties should toughen the polymers considerably. In addition, these monomers can be used as hard crosslinking segments for polyesters and epoxy composites to improve their mechanical and thermal properties. The development of these PET-based monomers functionalized with tertiary amines also provides the ability to form aromatic amides with water solubility. 
This trait is especially useful for interfacial polymerizations where the monomer can be dissolved in water, rather than more toxic solvents such as DMSO, to form high molecular weight polymers. Thus, despite the difficulties in isolating the tertiary-amine functionalized monomers, specifically PETDAMDPA, it is worth pursuing, as the other PET-based compounds do not share these solubility characteristics. The next chapter examines the ability to use a few of these PET monomers as hardeners for epoxy composites. 


\section{CHAPTER SIX}

\section{PET-DIAMINE RESULTS AS EPOXY HARDENING MODIFIERS}

The previous chapter focused on the development of a library of innovative monomers from PET waste using organocatalysis. The large diversity of PET-derived compounds suggests the potential for a diverse variety of applications. This thesis focuses primarily on the use of four PET-derived diamines (PETED, PETmX, PETDETA, and PETDAMDPA)(Figure 39) as epoxy hardening modifiers. As discussed in Chapter 4, epoxy composites are characterized using two different thermal characterization techniques: differential scanning calorimetry and dynamic mechanical analysis. Thermal characterization provides information on the typical thermal transitions that occur within thermosets. From the TTT diagram, we can characterize the effect of PET-diamines on the curing of epoxies as a modifier on the basis of their glass transition temperatures through the isothermal curing of each sample.

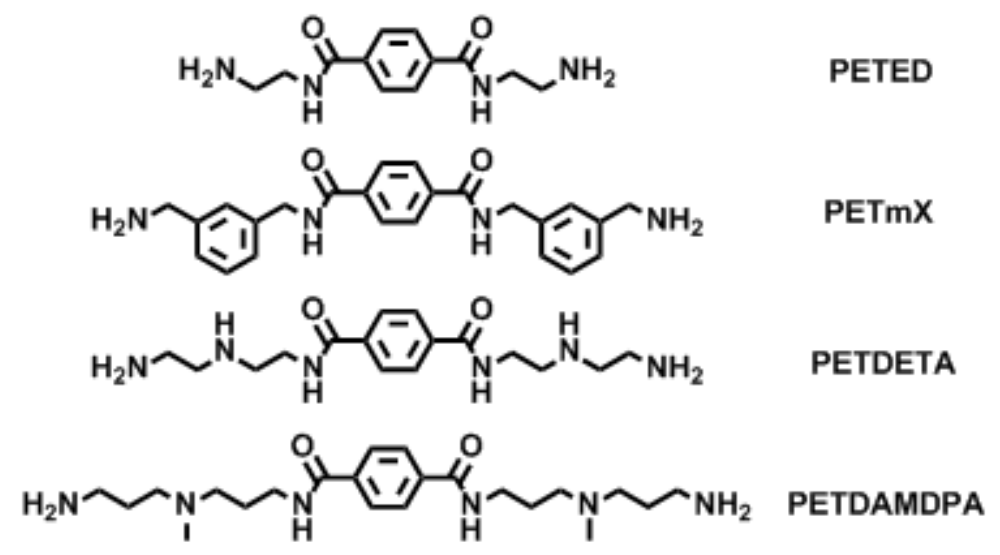

Figure 39. PET-derived compounds used as modifiers for epoxy hardening. 


\subsection{Differential Scanning Calorimeter Results}

The differential scanning calorimeter (DSC) measures the heat flow through the sample as a function of temperature. When curing PET-diamines with BAPDGE, the behavior of the specific heat of the composite as a function of temperature should be consistent with the curve typical for thermosets (Figure 24). The DSC analysis for curing BAPDGE with $100 \%$ equivalence of PETED is shown in Figure 40. The fact that the curve behaves identically to typical cured thermoset polymers demonstrates the ability for PET-diamines to successfully bind with the epoxy resin. This is also very important, as it demonstrates no significant influence from the solvent HFP on the curing process.

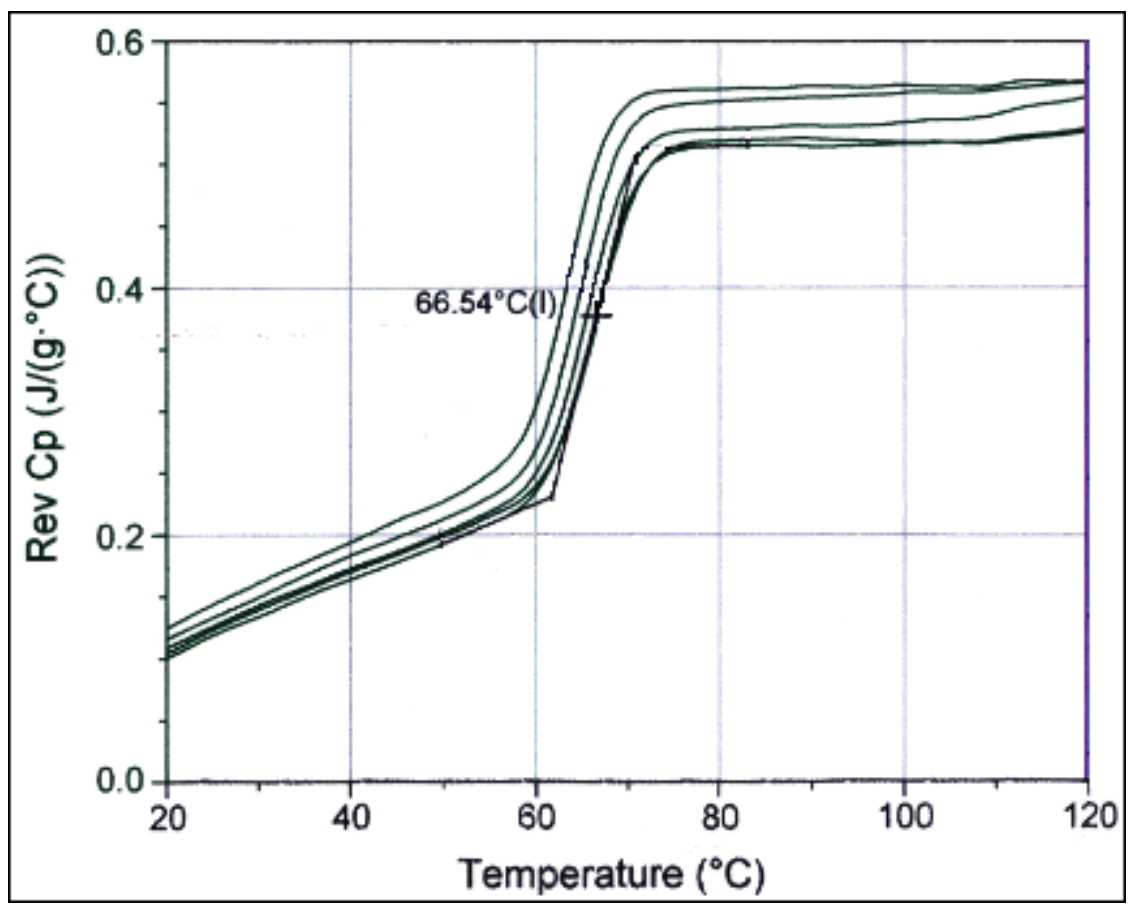

Figure 40. DSC analysis of cured epoxy polymer (100\% PETED) heated and cured several times to reach $\mathrm{Tg}_{\infty}$. 
The glass transition temperatures of each composite sample were estimated at the temperature where the largest change in specific heat occurred. However, as discussed previously, the epoxy composite did not always reach complete cross-linking after curing the sample at $200^{\circ} \mathrm{C}$ for 2 hours. Therefore, it was necessary to heat and cool the sample several times in the DSC in order to find the value of the glass transition temperature at the fully cured state $\left(\mathrm{Tg}_{\infty}\right)$. The $\mathrm{Tg}$ values for each heating/cooling, as illustrated in Figure 40, were relatively constant (only $1-2^{\circ} \mathrm{C}$ difference), demonstrating that the sample reached full cure using the given curing conditions $\left(200^{\circ} \mathrm{C}\right.$ for 2 hours $)$.

Using the DSC, the $\mathrm{Tg}_{\infty}$ of different compositions of PET-derived diamines $(0 \%$, $30 \%, 50 \%, 70 \%$, and 100\%) were obtained. As shown in Figure 41, higher compositions of PET-diamines in the epoxy curing process generally demonstrated higher $\mathrm{Tg}_{\infty}$ over their simple counterparts. The positive trend of the Tg as a function of PET-monomer composition can be explained by the added structural rigidity and hydrogen bonding effects from the aromatic amide on the PET-derived monomers. These effects caused the intermolecular forces between the cross-linked chains to be significantly stronger, thus requiring more energy to loosen the bonds as the composite reached the glass transition state. However, for PETDAMDPA, the extent of $\mathrm{Tg}_{\infty}$ increasing was significantly lower than it was for the other PET-diamines. This can be attributed to the fact that PETDAMDPA is larger and more flexible because of the long aliphatic chains negating the effects of the aromatic amide groups. 


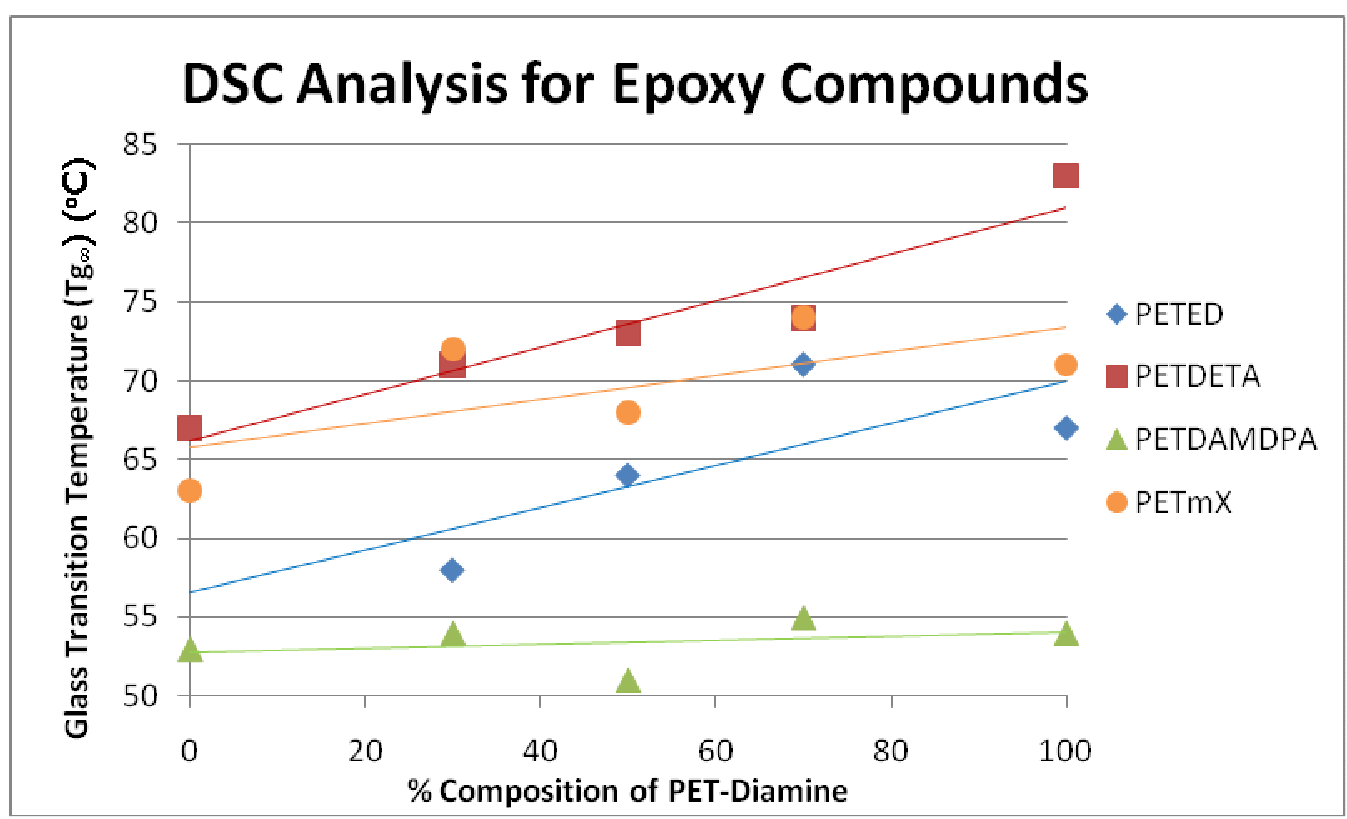

Figure 41. DSC analysis for epoxy hardened polymers using PET-derived diamines.

The $\mathrm{Tg}_{\infty}$ can also be compared for each PET-diamine based on their respective structures (Table 15). PETDETA, as expected, had the highest $\operatorname{Tg}_{\infty}\left(\sim 83^{\circ} \mathrm{C}\right)$ because it contains a free primary amine and secondary amine that can react with epoxide group of BAPDGE. As a result, the cross-linking density of the composite was considerably higher, making the composite significantly more rigid and requiring additional energy to allow cross-links to relax as the glass temperature was reached. As for the other three PET-derived diamines, the value of $\mathrm{Tg}_{\infty}$ depended mainly on the rigidity of the free amine. The free benzylamine on $\operatorname{PETmX}\left(\operatorname{Tg}_{\infty}=71^{\circ} \mathrm{C}\right)$ provided additional rigidity, making it slightly more difficult than PETED $\left(\mathrm{Tg}_{\infty}=67^{\circ} \mathrm{C}\right)$ and PETDAMDPA $\left(\mathrm{Tg}_{\infty}=\right.$ $54^{\circ} \mathrm{C}$ ) to relax as the composite reached the glass transition state. Additionally, the epoxy samples cured with PETDAMDPA required a lower Tg to allow the composite to reach the glass transition state, as the hydrogen bonding and rigidity effects were negated by 
the size and flexibility of PETDAMDPA. In conclusion, the DSC analysis provided a great deal of information on the thermal characteristics of the cured epoxy polymers based on the structural components of the curing agents.

Table 15. Table of $\mathrm{Tg}_{\infty}$ values of cure epoxy composite with $100 \%$ PET-diamine

\begin{tabular}{|c|c|}
\hline $\mathbf{1 0 0 \%}$ PET-diamine & $\mathbf{T g}_{\infty}\left({ }^{\circ} \mathbf{C}\right)$ \\
\hline PETED & 67 \\
\hline PETmX & 71 \\
\hline PETDETA & 83 \\
\hline PETDAMDPA & 54 \\
\hline
\end{tabular}

\subsection{Dynamic Mechanical Analysis Results}

Dynamic mechanical analysis provides another thermal characterization tool to describe the curing behavior between PET-derived diamines and epoxy resin. DMA provides thermal characterization of the desired composite by testing the storage modulus as a function of temperature. The Tg through DMA can be calculated by determining the point at which the modulus starts to drop into the elastomeric region. The glass transition temperatures determined through DMA were slightly different than the $\mathrm{Tg}_{\infty}$ determined through DSC, as the instruments used different phenomena to determine the point at which the composite reached the glass transition state. It is also important to note that the value of the modulus in the DMA was purely relative, since the dimensions of the composite cannot be accurately determined with the presence of the glass fiber. This 
section presents the thermal characterization results for the four PET-derived modifiers using DMA.

\subsubsection{PETED Composite DMA Results}

The first test conducted using DMA focused on the curing of the epoxy resin with PETED. Figure 42 illustrates the behavior of the modulus of the thermosets cured with $100 \%$ PETED and $100 \%$ ED as a function of temperature. The DMA results confirm that both monomers cured correctly with the epoxy resin, as the shape of the storage modulus curve (sinusoidal curve) followed that illustrated in Figure 26. From the DMA, the Tg of the thermosets cured with PETED and ED were $74^{\circ} \mathrm{C}$ and $51^{\circ} \mathrm{C}$, respectively. The DMA Tg values for the epoxy samples cured with PETED/ED were similar to the values calculated through DSC, further substantiating the $\mathrm{Tg}$ for the sample. It is also important to note that the tan delta peak for the PETED sample in comparison to the ED sample was rather broad. The cross-linking within the sample was not uniform and, as a result, showed a wide distribution of glass transition temperatures. This makes sense, as the bulky aromatic amide group in PETED should cause additional voids within the polymer. 
(a)

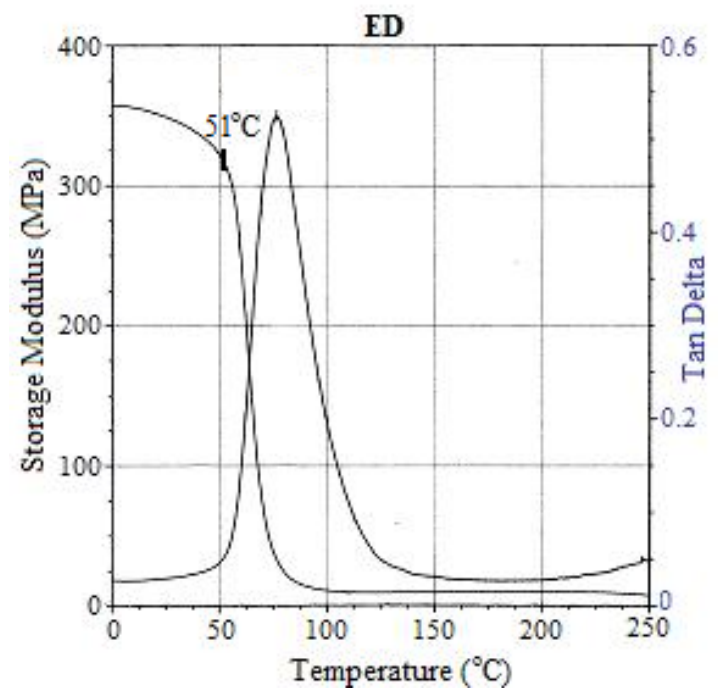

(b)

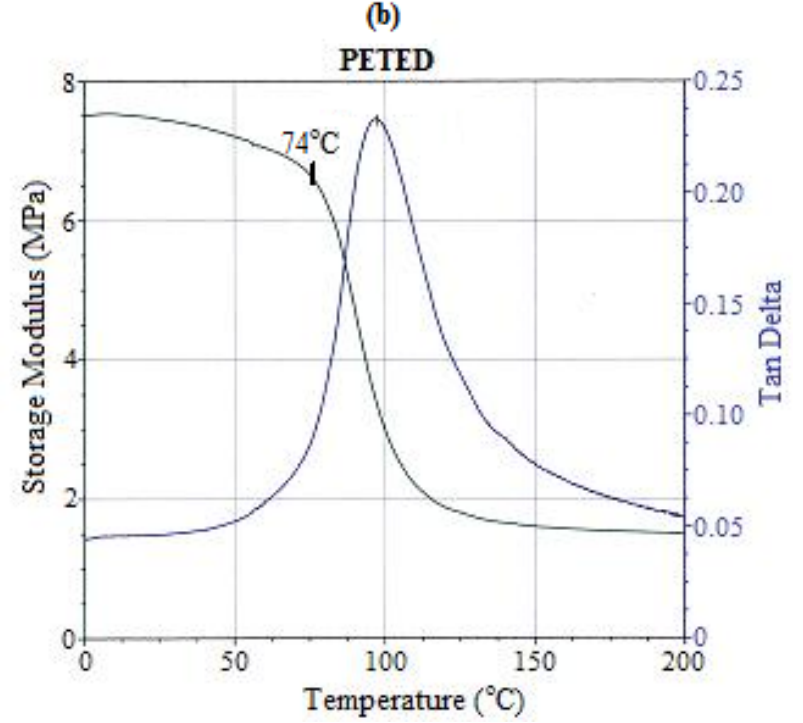

Figure 42. DMA analysis for epoxy curing with (a) Ethylenediamine and (b) PETEthylenediamine.

\subsubsection{PETmX Composite DMA Results}

The next dynamic mechanical analysis on the curing of epoxy resin with PETderived diamines was conducted on PETmX. Figure 43 illustrates the behavior of the modulus of the thermosets cured with $100 \%$ PETmX and $100 \% \mathrm{mX}$ as a function of temperature. The glass transition temperatures between the samples calculated through the DMA for the epoxy samples cured with $100 \%$ PETmX and the epoxy samples for the epoxy sample cured with $100 \% \mathrm{mX}\left(64^{\circ} \mathrm{C}\right.$ and $61^{\circ} \mathrm{C}$, respectively) deviated slightly from the values for the DSC. However, these values were reasonable, as they fell in the range of temperatures where the glass transition occured. The tan delta peak on the sample of PETmX also had a broader peak than the $\mathrm{mX}$ sample, which can be related to the steric effects of the aromatic amide on the cross-linking. However, the behavior of the storage 
modulus with the PETmX sample was flat before it reached the drop off point. A possible explanation for the deviation may be a slight phase separation that occurred when HFP evaporated out of solution. Therefore, there may be a few regions within the polymer that cured differently and caused the behavior of the modulus to change slightly.
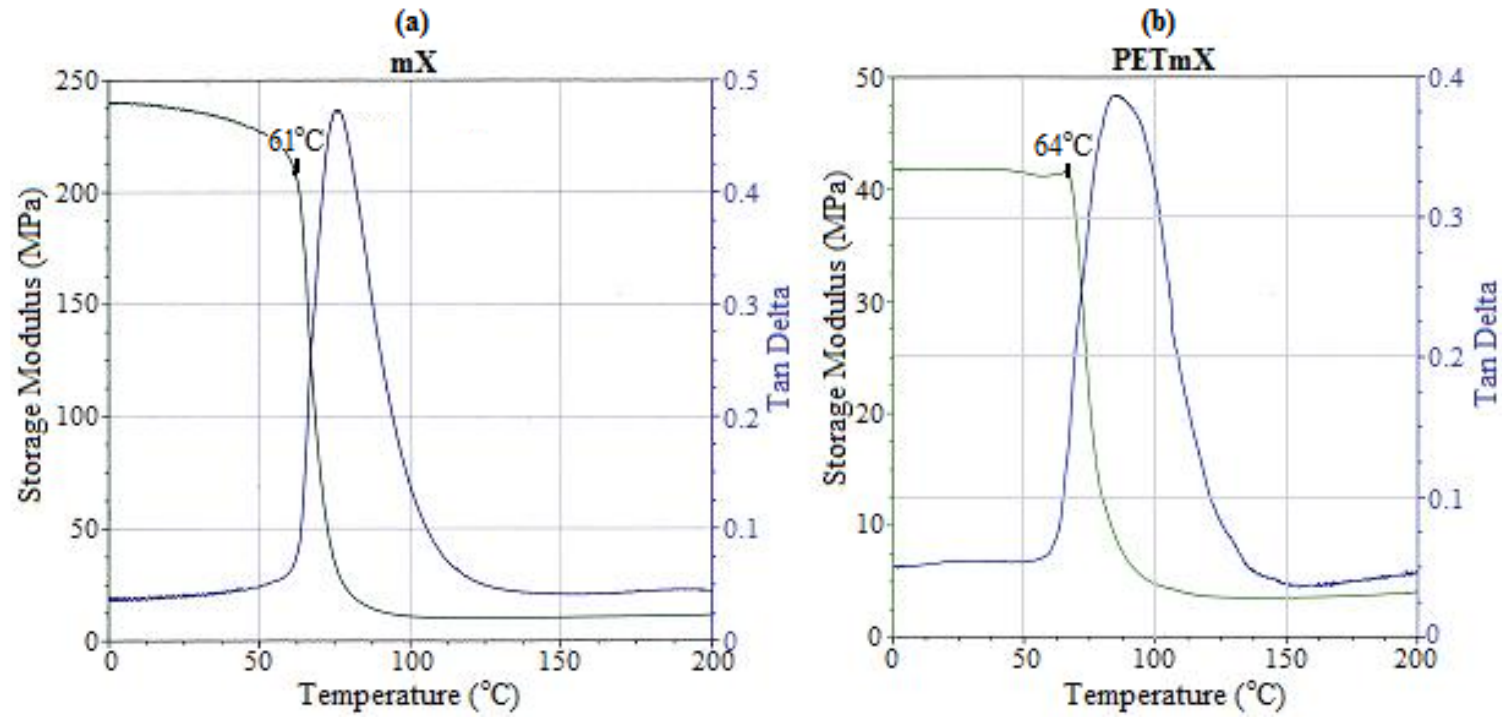

Figure 43. DMA analysis for epoxy curing with (a) m-xylylenediamine and (b) PET-mXylylenediamine. 


\subsubsection{PETDAMDPA Composite DMA Results}

The next DMA that was completed was on the curing behavior using

PETDAMPDA. Figure 44 illustrates the behavior of the modulus of the thermosets cured with $100 \%$ PETDAMDPA and $100 \%$ DAMDPA as a function of temperature. The DMA $\mathrm{Tg}$ values for the epoxy samples cured with PETDAMDPA $\left(53^{\circ} \mathrm{C}\right)$ matched the $\mathrm{Tg}$ values established from the DSC $\left(54^{\circ} \mathrm{C}\right)$. The samples cured with DAMDPA $\left(40^{\circ} \mathrm{C}\right)$, however, were significantly lower than the Tg values from the DSC $\left(53^{\circ} \mathrm{C}\right)$. There were some discrepancies in the curing behavior, as illustrated by the slight bump shown in the tan delta peak of the $100 \%$ DAMDPA sample in Figure 44. There may have been some slight phase separation that caused the non-uniform curing within the compound. Nonetheless, these Tg values still provide a reasonable picture of the thermal behavior of the epoxy composites of both samples. Another interesting observation is that the tan delta peaks for both samples were broader than those of the other PET-diamine/diamine samples. Because of the added flexibility provided by the PETDAMDPA/DAMDPA curing agents, one would expect the range of the glass transition temperatures to be wider due to the increasingly diverse amount of conformations that could occur between crosslinks. 
(a)

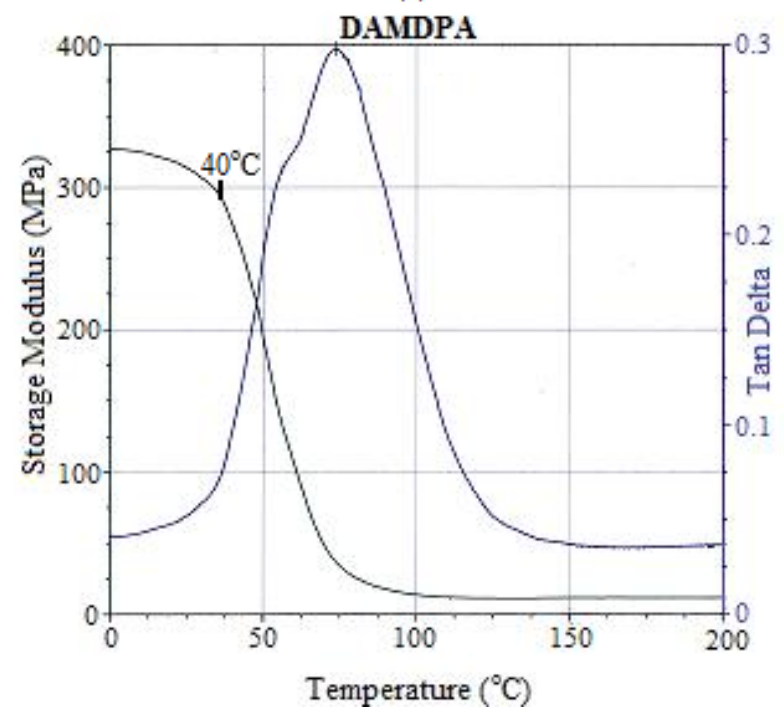

(b)

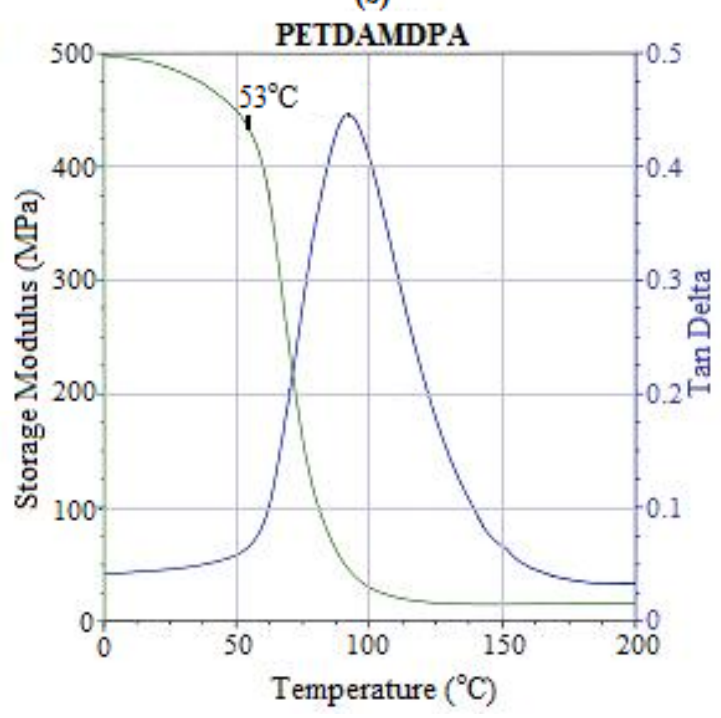

Figure 44. DMA analysis for epoxy curing with (a) Diamino-N-methyldipropylamine and (b) PET-Diamino-N-methyldipropylamine.

\subsubsection{PETDETA Composite DMA Results}

The final DMA was performed on the curing of the epoxy resin using PETDETA.

Figure 45 illustrates the behavior of the modulus of the thermosets cured with $100 \%$

PETDETA and 100\% DETA as a function of temperature. The glass transition behavior and broadness of the tan delta peak differed greatly from the other tested PET-based diamine samples. In this case, the tan delta peak was significantly narrower in comparison to the other PET-diamines. The additional secondary amine on the diethylenetriamine likely caused the polymer to have significantly higher cross-linking densities. As a result, the cross-linking should be drastically more uniform and more packed in comparison to the other PET-diamines. The glass transition temperature of the sample consisting of $100 \%$ PETDETA $\left(64^{\circ} \mathrm{C}\right)$ was actually significantly smaller than the $\mathrm{Tg}$ value determined from the $\operatorname{DSC}\left(83^{\circ} \mathrm{C}\right)$. One explanation could be that the extra 
spacing from the aromatic amide group would force the cross-linked chains to space around the bulky group and require less energy to stretch the sample, which is in a less constricted state when the load is applied. Through the DSC, no load was applied to the sample; therefore, the loosening of the bonds as the glass transition was reached occured only as a function of temperature. However, the $\mathrm{Tg}$ values calculated from the DSC fit in the glass transition range (Figure 45). Therefore, the Tg values determined from the DSC can still be accounted for and used to characterize the thermal behavior of the epoxy resin cured with PETDETA.

(a)

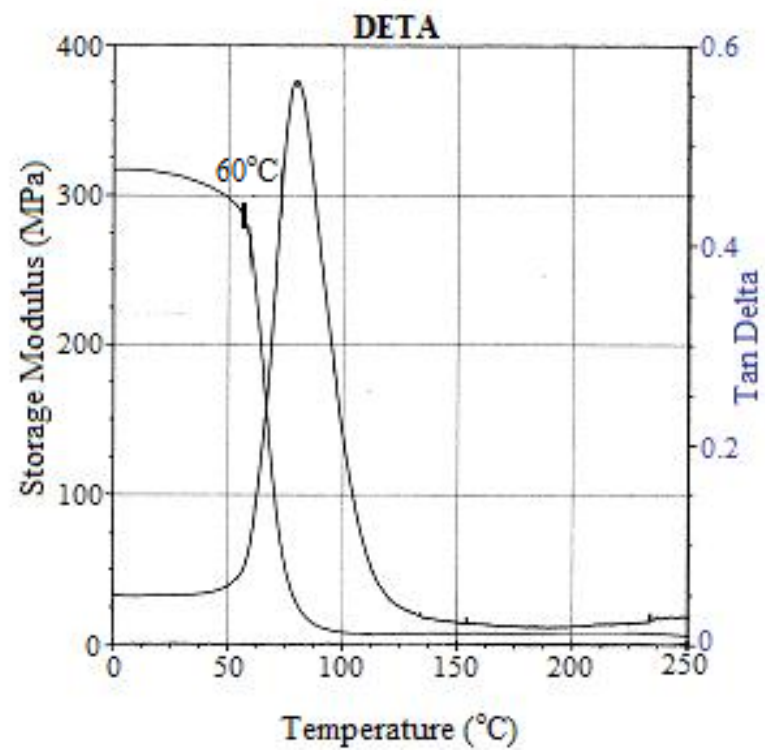

(b)

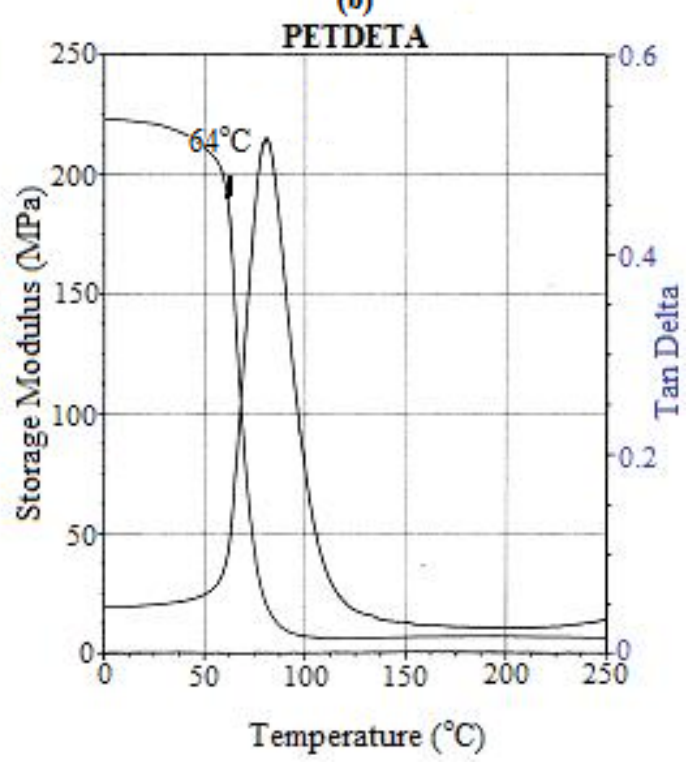

Figure 45. DMA analysis for epoxy curing with (a) Diethylenetriamine and (b) PETDiethylenetriamine. 


\section{CHAPTER SEVEN}

\section{CONCLUSIONS AND FUTURE WORK}

The growing concern regarding the overdevelopment of plastics, along with the inability to effectively eradicate current plastic waste products, has motivated the scientific community to develop innovative ways of solving this global problem. The focus of this project is to develop an innovative alternative to treating PET waste using organocatalysis. Using 1,5,7-triazabicyclo[4.4.0]dec-5-ene, a library of diverse monomers from PET waste bottles was developed with special properties for a variety of applications. In addition, the synthesis of these monomers was designed with an emphasis on green chemistry, as these reactions were carried out in low reaction conditions, as well as in the absence of solvent. As a result, PET aminolysis with aliphatic diamines and aromatic diamines provided the best results in comparison with the other amines tested due to the high reactivity of the reagents and the ease in isolating the products from the reagents. In addition, both m-xylylenediamine and ethylenediamine could be separated out and reused to produce additional PET monomers with impurities resulting from degraded TBD. PET degradation reactions with click functionalized amines and non-symmetric reagents produced the desired monomers with relatively low yields by targeting the reactive amine groups by altering the reaction temperature. Finally, PET degradation with secondary amines and tertiary functionalized 
amines yielded mixed results due to problems associated with the solubility between the reagents, products, and solvent.

Additional studies were done to demonstrate the ability to use PET-based monomers for additional high valued monomers, specifically epoxy hardening. Four different monomers (PETED, PETmX, PETDAMDPA, and PETDETA) were cured with the epoxy resin, bisphenol A propoxylate diglycidyl ether, using a polar solvent to dissolve the PET monomer. Each epoxy sample was thermally characterized using differential scanning calorimetry (DSC) and dynamic mechanical analysis (DMA) to determine the curing behavior. The DSC analysis of the epoxy composites cured with the PET-based monomers yielded higher glass transition temperatures than the composites cured with the reagent counterpart (e.g., PET-ethylenediamine vs. ethylenediamine) as a result of the added rigidity and hydrogen bonding effects obtained from the aromatic amide group present in the PET-based monomers. In addition, the Tg values for the epoxy samples cured with each of the PET-based monomers were strongly linked to the structural properties of the PET-derived monomer curing agents. The DMA results also confirmed the correct curing behavior for the samples cured with PET-based monomers, while generally yielding similar glass transition temperatures with those found from the DSC.

This thesis focuses specifically on the aminolysis of PET waste products for the development of structurally rigid, versatile monomers. However, current research is being done on the transesterification of PET waste with ethylene glycol to form bis(2hydroxyethyl) terephthalate, a monomer used to repolymerize into PET. Therefore, there 
is a lot of potential to develop similar monomers from PET waste through the transesterification of alcohols to form a library of aromatic esters. This thesis focuses on one particular application for these monomers: epoxy hardening. The extensive collection of PET-based monomers has various attractive properties that can be used for various other applications ranging from desalination membranes to the development of specialty monomers (polyamides and click polymers). The importance of this research is not only to develop a method of solving current problems, but to create new opportunities in research. 


\section{REFERENCES}

1) M. Babiker, J.M. Reilly, and H.D. Jacoby, "The Kyoto Protocol and developing countries," Energ. Policy, 28 (8), 525-536 (2000).

2) P.T. Anastas and M.M. Kirchhoff, "Origins, current Status, and future challenges of green chemistry,” Acc. Chem. Res., 35, 686-694 (2002).

3) S.J. Jeon, H. Li, and P.J. Walsh, "A green chemistry approach to a more efficient asymmetric catalyst: solvent-free and highly concentrated alkyl additions to ketones," J. Am. Chem. Soc., 127, 16416-16425 (2005).

4) Environmental Protection Agency, "Municipal solid waste generation, recycling, and disposal in the United States: facts and figures for 2007," Accessed at http://www.epa.gov/epawaste/nonhaz/municipal/pubs/msw07-fs.pdf (Accessed on April 4, 2009). PDF File.

5) Chemsystems Prep Program, "Polyethylene terephthalate," (2009) Available at http://www.chemsystems.com/reports/ (Accessed on April 9, 2009). PDF File.

6) National Association for PET Container Resources, "2007 report on post consumer PET container recycling activity (final report)," [Online]. Available at http://www.napcor.com/pdf/2007PET_Report.pdf (Accessed on April 4, 2009). PDF File.

7) L.M. Rios, C. Moore, and P.R. Jones, "Persistent organic pollutants carried by synthetic polymers in the ocean environment," Mar. Pollut. Bull., 54 (8), 12301237 (2007).

8) L. Ritter, K.R. Solomon, J. Forget, M. Stemeroff, and C. O'Leary (1995), "Persistent organic pollutants, an assessment report on: DDT-aldrin-dieldrinendrin-chlordane-heptachlor-hexachlorobenzene-mirex-toxaphene, polychlorinated biphenyls, dioxins and furans, " [Online]. Available at http://www.chem.unep.ch/Pops/ritter/en/ritteren.pdf (accessed 9 September 2009). PDF files.

9) J. Milgrom, "Polyethylene terephthalate (PET)," in Plastics Recycling: Products and Processes, (Hanser Gardner Publications. Cincinnati, OH, 1992) pp. 52-54.

10) H. Cornier-Rios, "Effect of recycling on material properties of polyethylene terephthalate at various recycling ratios and recycling generations," Thesis University of Puerto Rico (2003). 
11) A.H. Tullo, "From refuse to reuse," Chemical Engineering \& News, 85 (42), 1520 (2007).

12) D. Paszun and T. Spychaj. "Chemical recycling of poly(ethylene terephthalate," Ind. Eng. Chem. Res., 36 (4), 1373-1383 (1997).

13) S. Kobayashi, Y. Mori, and Y. Yamashita, "Metal-complexes as Lewis-acid catalysts in organic synthesis," Comp. Coor. Chem. II, 9 (8), 399-444 (2004).

14) N.E. Kamber, W. Jeong, R.M. Waymouth, R.C. Pratt, B.G.G. Lohmeijer, and J.L. Hedrick, "Organocatalytic ring-opening polymerization," Chem. Rev. 107, 58135840 (2007).

15) K. Ishihara, M. Nakayama, S. Ohara, and H. Yamamoto, "Direct ester condensation from a 1:1 mixture of carboxylic acids and alcohols catalyzed by hafnium(IV) or zirconium(IV) salts," Tetrahedron, 58 (41), 8179-8188 (2002).

16) J. Otton, S. Ratton, V.A. Vasnev, G.D. Markova, K.M. Nametov, V.I. Bakhmutov, L.I. Komarova, S.V. Vinogradova, and V.V. Korshak, "Investigation of the formation of poly(ethylene terephthalate) with model molecules: kinetics and mechanisms of the catalytic esterification and alcoholysis reaction. II. Catalysis by metallic derivatives (monofunctional reactants)," J. Polym. Sci. A1, 26, 2199-2224 (1988).

17) G.R.J. Thatcher and R. Kluger, "Mechanism and catalysis of nucleophilic substitution in phosphate esters," in Advances in Physical Organic Chemistry (Academic Press Lim., San Diego, California, 1989), pp. 109-112.

18) W. Steglich and G. Höfle, "N,N-dimethyl-4-pyridinamine, a very effective acylation catalyst," Agnew. Chem. Int. Ed., 8 (12), 981 (1969).

19) A.C. Spivey and S. Arseniyadis, "Nucleophilic catalysis by 4(dialkylamino)pyridines revisited - the search for optimal reactivity and specificity," Agnew. Chem. Int. Ed., 43, 5436-5441 (2004).

20) S. Xu, I. Held, B. Kempf, H. Mayr, W. Steglich, and H. Zipse, "The DMAPcatalyzed acetylation of alcohols - a mechanistic study," Chem. Eur. J., 11, 47514757 (2005).

21) K.A. Ahrendt, C.J. Borths, and D.W.C. MacMillan, "New strategies for organic catalysis: the first highly enantioselective organocatalytic Diels-Alder reaction," J. Am. Chem. Soc., 122, 4243-4244 (2000). 
22) G.W. Nyce, J.A. Lamboy, E.F. Connor, R.M. Waymouth, and J.L. Hedrick, "Expanding the catalytic activity of nucleophilic n-heterocyclic carbenes for transesterification reactions," Org. Lett., 4 (21), 3587-3590 (2002).

23) R. Singh, R.M. Kissling, M. Letellier, and S.P. Nolan, "Transesterification/acylation of secondary alcohols mediated by n-heterocyclic carbene catalysts," J. Org. Chem., 69, 209-212 (2004).

24) G.A. Grasa, R.M. Kissling, and S.P. Nolan, "N-heterocyclic carbenes as versatile nucleophilic catalysts for transesterification/acylation reactions," Org. Lett., 4 (21), 3583-3586 (2002).

25) T. Inokuma, Y. Hoashi, and Y. Takemoto, "Thiourea-catalyzed asymmetric Michael addition of activate methylene compounds to $\alpha, \beta$-unsaturated imides: Dual activation of imide by intra- and intermolecular hydrogen bonding," J. Am. Chem. Soc., 128, 9413-9419 (2006).

26) R.C. Pratt, B.G.G. Lohmeijer, D.A. Long, P.N.P. Lundberg, A.P. Dove, H. Li, C.G. Wade, R.M. Waymouth, and J.L. Hedrick, "Exploration, optimization, and application of supramolecular thiourea-amine catalysts for the synthesis of lactide (co)polymers," Macromolecules, 39 (23), 7863-7871 (2006).

27) G. Bouhadir, R.W. Reed, R. Réau, and G. Bertrand, "Synthesis of onio-, dionio-, and trionio-substituted phosphines; the nucleophilic behavior of DBN and DBU toward main group electrophiles," Heteroatom Chem., 6 (4), 371-375 (1995).

28) Y.J. Im, J.H. Gong, H.J. Kim, and J.N. Kim, "Nucleophilic behavior of DBU and DBN toward acetylated Baylis-Hillman adducts," Bull. Korean Chem. Soc., 22 (9), 1053-1055 (2001).

29) B.G.G. Lohmeijer, R.C. Pratt, F. Leibfarth, J.W. Logan, D.A. Long, A.P. Dove, F. Nederberg, J. Choi, C. Wade, R.M. Waymouth, and J.L. Hedrick, "Guanidine and amidine organocatalysts for ring-opening polymerization of cyclic esters," Macromolecules, 39 (25), 8574-8583 (2009).

30) A. Chuma, H.W. Horn, W.C. Swope, R.C. Pratt, L. Zhang, B.G.G. Lohmeijer, C.G. Wade, R.M. Waymouth, J.L. Hedrick, and J.E. Rice, "The reaction mechanism for the organocatalytic ring-opening polymerization of l-lactide using a guanidine-based catalyst: hydrogen-bonded or covalently bonded?’ J. Am. Chem. Soc., 130 (21), 6749-6754 (2008).

31) M.J. Green, “Transesterification of carboxylic or carbonic esters," U.S. Patent 4681967 (1987). 
32) M.J. Green, "Process for transesterification of carbonate esters and carboxylic acid esters using a cyclic amidine catalyst," U.S. Patent 4652667, (1987).

33) U. Schuchardt, R. Sercheli, and R.M. Vargas, "Transesterification of vegetable oils: a review,” J. Braz. Chem. Soc., 9 (3), 199-210 (1998).

34) R.C. Pratt, B.G.G. Lohmeijer, D.A. Long, R.M. Waymouth, and J.L. Hedrick, "Triazabicyclodecene: a simple bifunctional organocatalyst for acyl transfer and ring-opening polymerization of cyclic esters," J. Am. Chem. Soc., 128 (14), 45564557 (2006).

35) C. Sabot, K.A. Kumar, S. Meunier, and C. Mioskowski, "A convenient aminolysis of esters catalyzed by 1,5,7-triazabicyclo[4.4.0] dec-5-ene (TBD) under solventfree conditions," Tetrahedron Lett., 48, 3863-3866 (2007).

36) T. Spychaj, E. Fabrycy, S. Spychaj, and M. Kacperski, "Aminolysis and aminoglycolysis of waste poly(ethylene terephthalate)," J. Mater. Cycles Waste Manag., 3, 24-31 (2001).

37) J.B. Enns and J.K. Gillham, "Time-temperature-transformation (TTT) cure diagram: Modeling the cure behavior of thermosets," J. Appl. Polym. Sci., 28, 2567-2591 (1983).

38) A.J. Kinloch and R.J. Young, Fracture Behavior of Polymers, (Applied Science Publishers Ltd, London, England, 1983), pp. 1-37.

39) H.F. Mark, N.M Bikales, C.G. Overberger, and G. Menges, "Epoxy Resins," Encyclopedia of Polymer Science and Engineering, 6, edited by J. I. Kroschwitz (John Wiley \& Sons, New York, NY, 1986), pp. 322-356.

40) C.C. Riccardi, H.E. Adabbo, and R.J.J. Williams, "Curing reaction of epoxy resins with diamines," J. Appl. Polym. Sci., 29, 2481-2492 (1984).

41) P.D. McLean, A. Garton, R.F. Scott, and S.E. Gransden, "Epoxy resin fortifiers based on aromatic amides," U.S. Patent 4480082 (1984).

42) T. Iijima, N. Arai, W. Fukuda, and M. Tomoi, "Toughening of aromatic diaminecured epoxy resins by poly(ethylene phthalate)s and the related copolyesters," Eur. Polym. J., 31 (3), 275-284 (1995).

43) S.G. Hong and C.S. Wu, "DSC and FTIR analysis of the curing behavior of epoxy/DICY/solvent open systems," Thermochem. Acta, 316, 167-175 (1998). 
44) J.H. Bradbury, P.J. Crawford, and A.N. Hambly, "Kinetics of an interfacial polycondensation reaction. Part 2. - Reaction of terephthaloyl chloride with piperazine," Trans. Faraday Soc., 64, 1337-1347 (1968).

45) R.J. Petersen, "Composite reverse osmosis and nanofiltration membranes," J. Membrane Sci., 83, 81-150 (1993).

46) J.E. Cadotte, "Interfacially synthesized reverse osmosis membrane," U.S. Patent 4277344, (1981).

47) Z. Yong, Y. Sanchuan, L. Meihong, and G. Congjie, "Polyamide thin film composite membrane prepared from m-phenylenediamine and $m$ phenylenediamine-5-sulfonic acid," J. Membrane Sci., 270, 162-168 (2006).

48) J.P. Sheth, D.B. Klinedinst, G.L. Wilkes, I. Yilgor, and E. Yilgor, "Role of chain symmetry and hydrogen bonding in segmented copolymers with monodisperse hard segments," Polymer, 46, 7317-7322 (2005).

49) J.P. Sheth, D.B. Klinedinst, T.W. Pechar, G.L. Wilkes, E. Yilgor, and I. Yilgor, "Time-dependent morphology development in a segmented polyurethane with monodisperse hard segments based on 1,4-phenylene diisocyanate," Macromolecules, 38, 10074-10079 (2005).

50) W.H. Binder and R. Sachsenhofer, “' 'Click' chemistry in polymer and material science: An update," Macromol. Rapid Comm., 29, 952-981 (2008).

51) A.K. Feldman, B. Colasson, and V.V. Fokin, "One-pot synthesized of 1,4disubstitued 1,2,3-triazoles from in-situ generated azides," Org. Lett., 6 (22), 3897-3899 (2004).

52) L. Feller, "PET and Intermediates," Chemical Industry News and Intelligence, [Online]. Available at http://icispricing.com/il_shared/Samples/SubPage177.asp (Accessed January 31, 2010). HTML File.

53) J. Chan, "Mono Ethylene Glycol (Asia Pacific)," Chemical Industry News and Intelligence [Online]. Available at http://icispricing.com/il_shared/Samples/SubPage188.asp (Accessed January 31, 2010). HTML File.

54) Just-in-time Lab Supplies, "Triethylenetetramine, Purified, 4x4L Amber Glass," Available at http://www.jitlab.com/product.php?productid=24117\&cat=1147\&page $=1$ (Accessed January 31 2010). HTML File. 
55) H. Finch, "Ethylene Diamine (Europe)," Chemical Industry News and Intelligence [Online]. Available at http://icispricing.com/il_shared/Samples/SubPage111.asp (Accessed February 4, 2010). HTML File.

56) H. Xie, "Supply m-Xylylenediamine," [Online]. Available at http://www.echinachem.com/en/products/Product.aspx?SupplyOrRequestID=583 $\underline{013}$ (Accessed February 4, 2010). HTML File. 